\title{
Relationships between capnogram parameters and respiratory mechanics in ventilated patients
}

\author{
Zsófia Csorba, MD \\ $\mathrm{PhD}$ Thesis \\ Department of Anaesthesiology and Intensive Therapy \\ University of Szeged, Hungary \\ Doctoral School of Multidisciplinary Medical Sciences
}

Supervisor: Barna Babik MD, PhD

Szeged

2016 


\section{ABSTRACT}

Capnography is one of the most frequently used monitoring methods in anaesthesia and intensive therapy. However, details as to how the resistive and/or elastic properties of the respiratory system affect the various indices derived from the capnogram curve are lacking from the literature. The aims of the present thesis were therefore to establish the connections between the various phase, shape, dead space or pulmonary shunt circulation parameters of the time or volumetric capnogram and those reflecting the airway and respiratory tissue mechanics, expiratory flow and gas exchange.

A large cohort of patients scheduled for elective cardiac surgery was enrolled in this thesis. After induction of total intravenous anaesthesia, the patients were intubated and ventilated. Forced oscillation technique was applied to measure airway resistance (Raw), tissue damping $(\mathrm{G})$ and elastance $(\mathrm{H})$. Time and volumetric capnography were performed to assess parameters reflecting the phase II $\left(\mathrm{S}_{\mathrm{II}}\right)$ and III slopes $\left(\mathrm{S}_{\mathrm{III}}\right)$, their transition $\left(\mathrm{D}_{2 \mathrm{~min}}\right)$, and the deadspace indices according to Fowler, Bohr and Enghoff approach. The respiratory resistance (Rrs) and the dynamic compliance (Crs) displayed by the ventilator were registered, and arterial and central venous blood gas analysis were performed. In the first study (Study 1) the measurement was performed in open-chest condition before and $5 \mathrm{~min}$ after cardiopulmonary bypass (CPB), whereas in the second study (Study 2) of this thesis, the measurements were accomplished at positive end-expiratory pressure (PEEP) levels of 3,6 and $9 \mathrm{~cm} \mathrm{H}_{2} \mathrm{O}$ in patients with healthy lungs, and in patients with respiratory symptoms involving low (Group LC), medium (Group MC) or high Crs (Group HC).

In Study $1, \mathrm{~S}_{\mathrm{II}}$ and $\mathrm{D}_{2 \min }$ exhibited the closest associations with $\mathrm{H}(0.65$ and 0.57 ; $\mathrm{p}<0.0001$, respectively), whereas $\mathrm{S}_{\text {III }}$ correlated most strongly with Raw $(r=0.63 ; \mathrm{p}<0.0001)$ before $\mathrm{CPB}$, whereas significant elevations in Raw and G, with smaller but still significant increases in $\mathrm{H}$ were induced by CPB. These adverse mechanical changes were reflected consistently in $S_{\text {II }}, S_{\text {III }}$ and $D_{2 m i n}$, with weaker correlations with the dead-space indices. The intrapulmonary shunt expressed as the difference between the Enghoff and Bohr dead-space parameters was increased after CPB $(95 \pm 5 \%$ vs. $143 \pm 6 \%$; $<<0.001)$. The results confirm that the capnographic parameters from the early phase of expiration $\left(S_{\text {II }}\right.$ and $\left.D_{2 m i n}\right)$ are linked to the pulmonary elastic recoil, while the effect of airway patency on $S_{\text {III }}$ dominates over the lung tissue stiffness in mechanically ventilated patients. However, severe deteriorations in lung resistance or elastance affect both capnogram slopes.

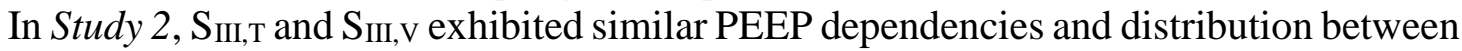
the protocol groups formed on the bases of Crs. A wide inter-individual scatter was observed in the overall Raw-SIII,V relationship, which was primarily affected by Crs. Decreases in Raw with increasing PEEP were reflected in sharp falls in $\mathrm{S}_{\text {III }}$ in Group HC, whereas $\mathrm{S}_{\text {III,T }}$ was insensitive to changes Raw in Groups LC and HL. In Group HC, $S_{\text {III }}$ was the highest and the oxygenation was similar than in the healthy group, in Group LC, the $S_{\text {III }}$ was similar than that in the healthy patients, but the oxygenation was the worst. According to our data, S SII provide meaningful information about alterations in airway caliber, but only within an individual patient. The sensitivity of $\mathrm{S}_{\text {III,T }}$ depends on Crs. Thus, assessment of the capnogram shape should always be coupled with Crs when the airway resistance or oxygenation are evaluated.

In conclusion, evaluation of the relationship between capnography and respiratory mechanics help the anaesthesiologist and intensive therapist to have a deeper understanding of the shape indices of the capnogram, and in a broad sense, to bridge the gap between the physiological and the clinical knowledge in adequate bedside monitoring. 


\section{List of scientific papers included in this thesis}

I. Csorba Z, Petak F, Nevery K, Tolnai J, Balogh AL, Rarosi F, Fodor GH, Babik B. Capnographic parameters in ventilated patients: correspondence with airway and lung tissue mechanics. Anesth \& Analg (Accepted for publication) [IF: 3.472]

II. Babik B, Csorba Z, Czövek D, Mayr PN, Bogáts G, Peták F. Effects of respiratory mechanics on the capnogram phases: importance of dynamic compliance of the respiratory system. Crit Care. 16(5):R177, 2012 [IF: 4.718]

\section{List of scientific papers related to this thesis}

I. Csorba Zs, Czövek D, Bogáts G, Peták F, Babik B. A légzésmechanika hatása a kapnogramra: a légzőrendszer elasztikus tulajdonságának jelentősége. Aneszteziológia és Intenzív Terápia 42(2): 77-84, 2012.

II. Babik B, Csorba Zs, Balogh Á, Szeti K, Tolnai J, Peták F. Kapnográfia lélegeztetett betegekben. Mindig nézzük, mindent látunk? Medicina Thoracalis LXVII:(2) pp. 78-98, 2014.

III. Balogh AL, Petak F, Fodor GH, Tolnai J, Csorba Zs, Vigh E, Nevery K, Babik B. Capnogram slope and ventilation dead space parameters: comparison of mainstream and sidestream techniques. Brit J Anaesth, 2016 (under review). 


\section{Table of contents}

List of scientific papers included in this thesis .............................................................. 2

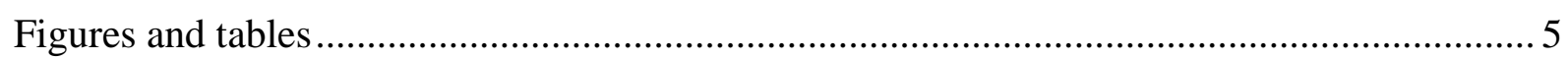

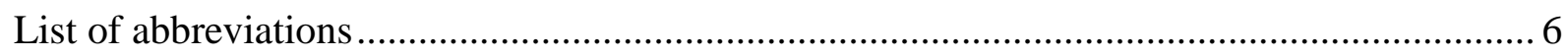

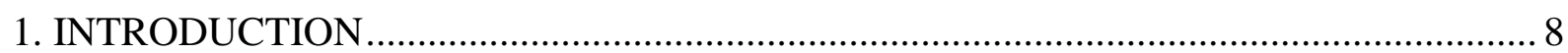

1.1. Monitoring of the ventilated patient: remarkable physical and physiological diversity . 8

1.2. Capnography: the familiar yet unknown monitoring modality ..................................... 8

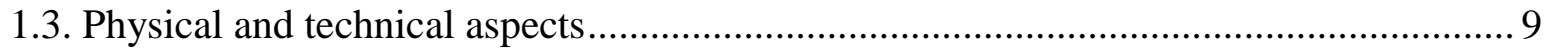

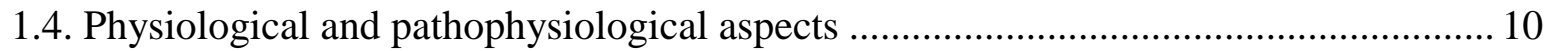

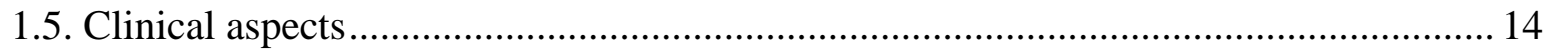

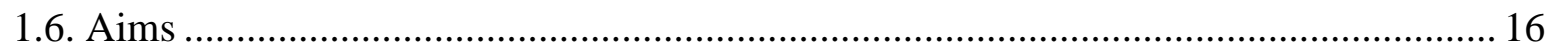

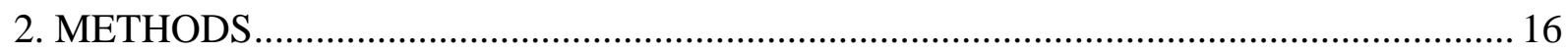

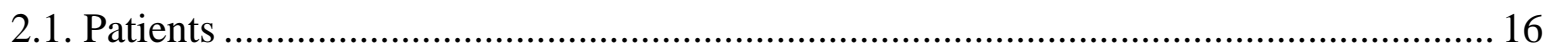

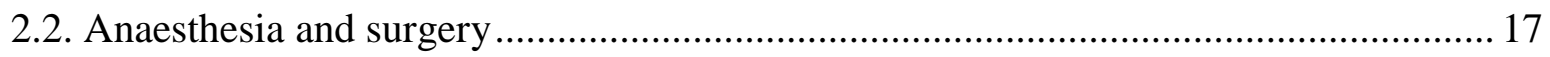

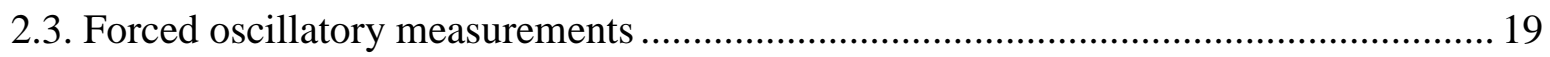

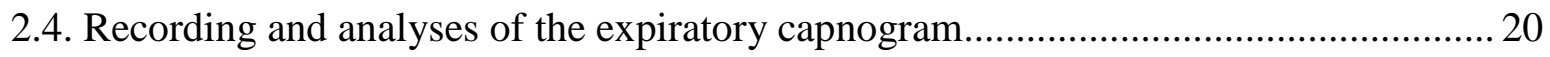

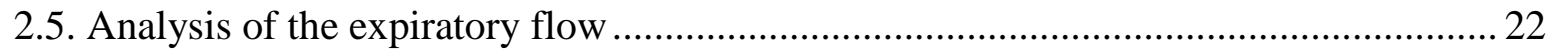

2.6. Calculating intrapulmonary shunt based on Fick principle and classic shunt equation 23

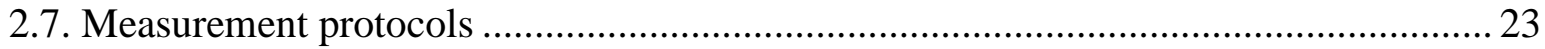

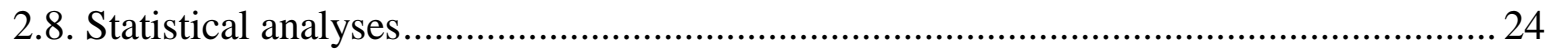

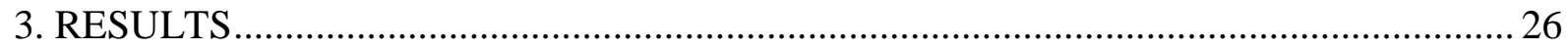

Study 1: Capnographic parameters: correspondence with airway and tissue mechanics ..... 26

Study 2: Respiratory mechanics and the capnogram phases: importance of dynamic

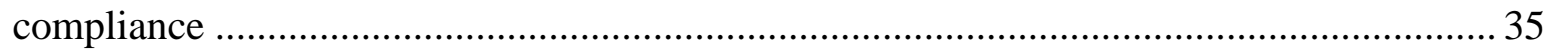

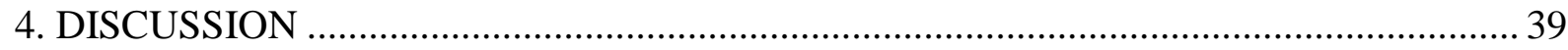

Study 1: Capnographic parameters: correspondence with airway and tissue mechanics..... 40 
Study 2: Respiratory mechanics and the capnogram phases: importance of dynamic compliance

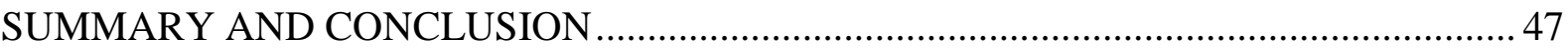

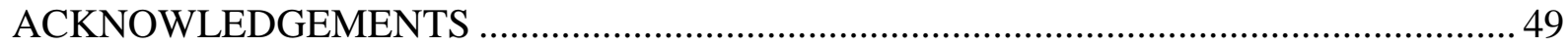

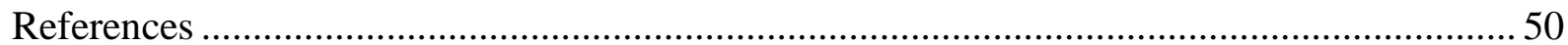




\section{Figures and tables}

Figure 1. Shape factors and characteristic partial pressures derived from the time and volumetric capnograms.

Figure 2. Timeline of the experimental protocol in Study 2.

Figure 3. Changes in resistive, compliance and expiratory flow parameters after CPB in Study 1 .

Figure 4. Changes in indices derived from the time and volumetric capnograms after CPB in Study 1 .

Figure 5. Strengths of the correlations between the lung mechanical and capnographic parameters in Study 1.

Figure 6. Strengths of correlations between fundamental lung mechanical and capnographic parameters in Study 1.

Figure 7. The key parameters obtained by forced oscillations and capnography for the individual patients and for the group means in Study 1.

Figure 8. The correlation of the time capnographic shape factors and the lung mechanical parameters in Study 1.

Figure 9. Changes in respiratory mechanical and capnographic parameters and $\mathrm{PaO}_{2}$ after increasing PEEP in Study 2.

Figure 10. Relationship between Raw and $S_{\text {III,T }}$ at increasing PEEP levels in Study 2.

Figure 11. Effects of BMI and EF on Crs in Study 2.

Table 1. The characteristics of the patients in each protocol group in Study 2.

Table 2. Coefficients of variations for the lung mechanical and capnographic parameters in Study 1 . 


\section{List of abbreviations}

$\mathrm{BMI}$
$\mathrm{CL}$
$\mathrm{CO}_{2}$
$\mathrm{COPD}$
$\mathrm{CPB}$
$\mathrm{Crs}$
$\mathrm{CtaO}_{2}$
$\mathrm{CtcO}_{2}$
$\mathrm{CtvO}_{2}$
$\mathrm{C}_{\text {vent }}$
$\mathrm{D}_{2 \text { min }}$

$\mathrm{D}_{2 \mathrm{Vmin}}$

EF

$\mathrm{FEV}_{1}$

$\mathrm{FiO}_{2}$

FRC

G

Group HC

\section{Group HL \\ Group LC}

Group MC

$\mathrm{H}$

HQ

Iaw

IV

content

content

domain

ventricle

Study 2
Body mass index

Total lung compliance

Carbon dioxide

Chronic obstructive

pulmonary disease

Cardiopulmonary bypass

Dynamic respiratory

compliance

Arterial oxygen content

Pulmonary capillary oxygen

Mixed venous oxygen

Compliance displayed by

the ventilator

Transition rate of $\mathrm{CO}_{2}$

concentration rate from

phase II to phase III in time

Transition rate of $\mathrm{CO}_{2}$

concentration rate from

phase II to phase III in

volume domain

Ejection fraction of left the

Expiratory volume in the

first second of forced

expiration

Fraction of inspired oxygen

Functional residual capacity

Respiratory tissue damping

Group of patients with high

dynamic respiratory

compliance in Study 2

Group of patients with

healthy lungs in Study 2

Group of patients with low

dynamic respiratory

compliance in Study 2

Qt

Raw

RL

Rrs

Rti

$\mathrm{R}_{\text {vent }}$

$S_{\text {II }}$

$S_{\text {III }}$

$\mathrm{S}_{\text {III,T }}$

$\mathrm{S}_{\mathrm{II}, \mathrm{V}}$

$\mathrm{S}_{\text {III,T }}$

Group of patients with

medium dynamic

respiratory compliance in

Respiratory tissue elastance

Horowitz coefficient

Airway inertance

Intravenous

LF

$\mathrm{PaCO}_{2}$

$\mathrm{PaO}$
$\mathrm{PaO}_{2}$

PEEP

$\mathrm{P}_{\overline{\mathrm{E}} \mathrm{CO}_{2}}$

PF

$\mathrm{Sn}_{\text {II,T }}$

$\mathrm{Sn}_{\text {II,V }}$

$\mathrm{Sn}_{\text {III,T }}$
IPPV

$\mathrm{P}_{\mathrm{ACO}_{2}} \quad$ Partial pressure of alveolar

$\mathrm{CO}_{2}$ concentration

$\mathrm{P}_{\mathrm{ETCO}_{2}}$

Qs Intrapulmonary shunted

fraction of the cardiac

output

Qs/Qt The fraction of the intrapulmonary shunt and the total intrapulmonary

blood flow

Total cardiac output

Airway resistance

Total lung resistance

Total respiratory resistance

Tissue resistance

Total respiratory resistance displayed by the ventilator

Capnogram phase II slope

Capnogram phase III slope

Time capnogram phase II

slope

Volumetric capnogram

phase II slope

Time capnogram phase III

slope

S III,V Volumetric capnogram

phase III slope
Normalized time capnogram phase II slope

Normalized volumetric capnogram phase II slope Normalized time capnogram phase III slope 


\begin{tabular}{|c|c|}
\hline $\mathrm{Sn}_{\text {III,V }}$ & $\begin{array}{l}\text { Normalized volumetric } \\
\text { capnogram phase III slope }\end{array}$ \\
\hline SIRS & $\begin{array}{l}\text { Systemic inflammatory } \\
\text { response syndrome }\end{array}$ \\
\hline tCap & Time capnogram \\
\hline vCap & Volumetric capnogram \\
\hline $\mathrm{V}^{\prime}$ & Tracheal airflow \\
\hline $\mathrm{V}^{\prime} \mathrm{CO}_{2}$ & $\begin{array}{l}\text { The amount of the excreted } \\
\mathrm{CO}_{2} \text { in } 1 \text { minute }\end{array}$ \\
\hline $\mathrm{V}_{\mathrm{DF}}$ & Fowler dead space \\
\hline $\mathrm{V}_{\mathrm{DB}}$ & Bohr dead space \\
\hline $\mathrm{V}_{\mathrm{DE}}$ & Enghoff dead space \\
\hline V'pl & $\begin{array}{l}\text { Plateau flow before the } \\
\text { beginning of the next } \\
\text { inspiration }\end{array}$ \\
\hline $\mathrm{V}_{\mathrm{T}}$ & Tidal volume \\
\hline ZL & $\begin{array}{l}\text { Input impedance of the } \\
\text { pulmonary system }\end{array}$ \\
\hline Zrs & $\begin{array}{l}\text { Input impedance of the } \\
\text { respiratory system }\end{array}$ \\
\hline$\alpha_{\text {cap }}$ & $\begin{array}{l}\text { Angle formed by the phase } \\
\text { II and phase III limbs of } \\
\text { time capnogram }\end{array}$ \\
\hline
\end{tabular}




\section{INTRODUCTION}

\subsection{Monitoring of the ventilated patient: remarkable physical and physiological diversity}

The bedside estimation of respiratory mechanics, monitoring of in-, and exhaled gas concentrations, assessing of gas exchange, or the different imaging technologies require a broad spectrum of low and high technology. Additionally, the information gained by these techniques about the ventilation, the ventilation perfusion mismatch, the cardiopulmonary interaction, the inflammatory reactions etc. exhibits an impressive physiological and pathophysiological variety.

The basic principles of patient's monitoring must be followed to find the balance in this diversity. The different parameters must always be compared and analysed, trends need to be made continuously, only monitoring methods with therapeutic consequences have to be applied, and all parameters must be summarized periodically. Appropriate bedside monitoring perfectly demonstrates, that healing the patient is not only an a priori process along with guidelines. To care a patient is rather a goal-directed procedure based on monitoring feedback, data analysing and human thinking with a strong effort to search for the agreement or the best compromise between the individual patient and the rather uniform principles of guidelines.

\subsection{Capnography: the familiar yet unknown monitoring modality}

Capnography is a non-invasive, continuous, on-line, dynamic, effort- and cooperationindependent, numeric and graphic bedside method for monitoring of the exhaled carbon dioxide $\left(\mathrm{CO}_{2}\right)$ concentration. The technique is one of the most frequently used monitoring methods in anaesthesia and intensive therapy, because capnography is able to detect vital signs during intentional temporary life-threatening alterations of vital functions with fast responses. However, pathophysiological and clinical information provided by the capnography has not yet been completely elucidated. 


\subsection{Physical and technical aspects}

The principle of the capnography is based on different light absorption abilities of materials. It was originally discovered by P. Bouguer in 1729 (1) and later only cited by J.H. Lambert in 1760 (2), then completed by A. Beer in 1852 (3). The Beer-Lambert law states that light absorbance of a material is proportional to the concentration and thickness of a sample. During capnography, emitted infrared light of $4.3 \mu \mathrm{m}$ wavelength is absorbed selectively by the exhaled $\mathrm{CO}_{2}$ (4).

According to the localization of the gas sampling method, capnography can be divided into mainstream and sidestream techniques. The essential part of the mainstream technique is a cuvette with a sampling window inserted into the ventilator circuit at the Ypiece to measure the $\mathrm{CO}_{2}$ concentration. This "near the patient" position has an advantage of rapid and exact signal processing $(5,6)$, but it also extends ventilation death space (7); it can exert traction forces to the endotracheal tube; it may obstruct head-neck surgery; it measures only the concentration of $\mathrm{CO}_{2}$ from the gas mixture, and needs to be continuously heated to avoid vapour precipitation (8).

Conversely, sidestream analysers take gas samples with a continuous suction rate from the same point of the ventilator circuit through a standard, $3 \mathrm{~m}$ long, small-diameter tube $(5,6)$. The $\mathrm{CO}_{2}$ concentration analysis is performed in a sample cell within the monitor, i.e. far from the patient. The obvious advantage of the sidestream technique is that it can easily be applied even during spontaneous ventilation throughout the perioperative period, and it is also able to measure the concentration of other gases, such as volatile anaesthetic agents. However, the sidestream method has also important disadvantages: condensation from humidified gas and secretions of the patient can block and contaminate the sampling line (6), and the continuous suction serves as a leakage and may pollute the operating theatre (9). Sidestream technique has a transit delay resulting in an axial gas mixing $(5,10,11)$, which causes a decrease in the dynamic response time of the sidestream capnographs $(5,11)$. In addition, sidestream technique underestimates the partial pressure of end-tidal $\mathrm{CO}_{2}$ concentration $\left(\mathrm{P}_{\mathrm{ETCO}_{2}}\right)$ at high ventilation frequencies $(\geq 30 / \mathrm{min})(12)$.

In the routine clinical practice, the concentration of $\mathrm{CO}_{2}$ is most frequently displayed in time domain (tCap). The didactic $\mathrm{CO}_{2}$-time curve is easy to interpret, because most of the 
vital monitoring parameters are depicted in time domain perioperatively. This form does not need any "second deduction step" during a systematic, rapid, repetitive, regular surveillance by any anaesthesiological team person $(4,13-16)$. The tCap can also be applied to monitor the spontaneous breathing patients, especially with the sidestream technique. The tCap has some disadvantages. The difference between the arterial $\left(\mathrm{PaCO}_{2}\right)$ and end-tidal $\mathrm{CO}_{2}$ can only be roughly estimated, because any non-sequential dead space increases, and any sequential dead space decreases the difference between $\mathrm{PaCO}_{2}$ and $\mathrm{P}_{\mathrm{ETCO}_{2}}$. Consequently, with tCap, the alveolar dead space indices and the amount of the excreted $\mathrm{CO}_{2}\left(\mathrm{~V}^{\prime} \mathrm{CO}_{2}\right)$ cannot be calculated (17).

Attaching flowmeter to the mainstream capnograph at the $\mathrm{CO}_{2}$ sampling point and derivating flow to volume data permits us to plot $\mathrm{CO}_{2}$-concentration in the volume domain within each respiratory cycle (vCap). The within-breath changes in $\mathrm{CO}_{2}$ concentration can be considered as a special single breath test curve, where the indicator gas is the endogenous $\mathrm{CO}_{2}$. vCap is increasingly available in sophisticated mechanical ventilators and/or anaesthesia monitors, which can provide relevant quantitative information about effective ventilation and $\mathrm{CO}_{2}$ elimination (18-23).

\subsection{Physiological and pathophysiological aspects}

Understanding the meaning of the phase and shape indices of normal and pathological time and volumetric capnogram are inevitable to interpret the curve and data appropriately.

From a morphological point of view, the expiratory part of the physiological capnogram curve can be divided into phase I, phase II and phase III. The relationship between the phase II and phase III is characterised by the angle $\alpha\left(\alpha_{\text {cap }}\right)$, and the direct transition from phase II to phase III can be described by the sharp or blunt shape of this capnogram region, signed as $\mathrm{D}_{2 \min }$ and $\mathrm{D}_{2 \mathrm{Vmin}}$ (Fig. 1 top and bottom) (24). As the expiratory flow decreases exponentially, the $\mathrm{CO}_{2}$ curve differs depending on whether the time or the volume domain was applied to plot the concentration of the exhaled $\mathrm{CO}_{2}$. After detailing the morphology of physiological tCap curve, all the differences between the tCap and vCap techniques are specified below. 

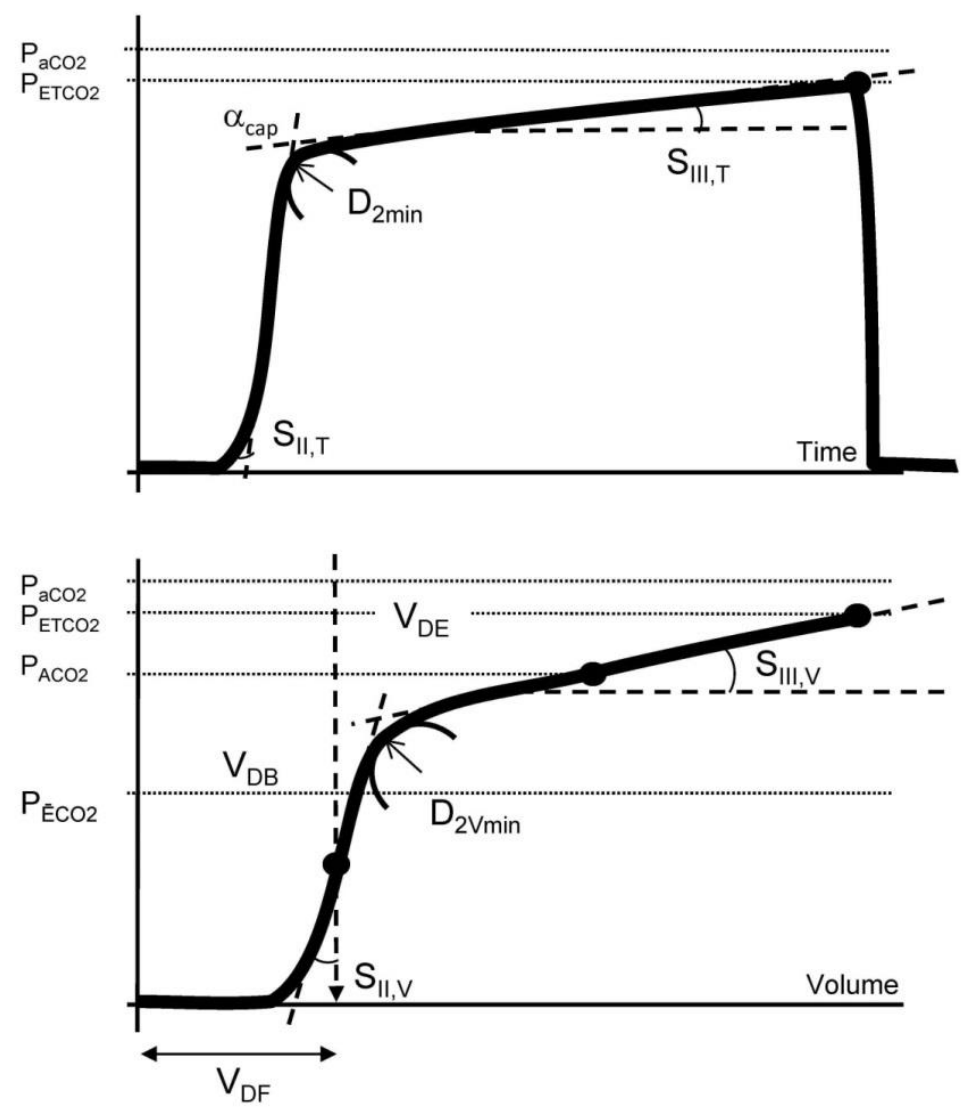

Figure 1. Shape factors and characteristic partial pressures derived from the time (top) and volumetric (bottom) capnograms. $\mathrm{PaCO}_{2}$ : partial pressure of arterial blood $\mathrm{CO}_{2} ; \mathrm{PACO}_{2}$ : mean alveolar $\mathrm{CO}_{2}$ concentration at the midpoint of phase III of $\mathrm{CO}_{2}$ expiration; $\mathrm{PECO}_{2}$ : mixed partial pressure of $\mathrm{CO}_{2}$ during the entire expiration; $\mathrm{PETCO}_{2}$ : end-tidal $\mathrm{CO}_{2}$ concentration; $\mathrm{S}_{\mathrm{II}} \mathrm{T}$ and $\mathrm{S}_{\mathrm{II}} \mathrm{V}$ : slopes of phase

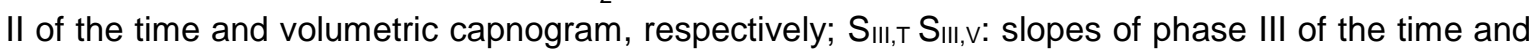
volumetric capnogram, respectively; $D_{2 m i n}$ and $D_{2 v m i n}$ : curvature at transition the phase II-III, calculated as the minimum of the second-order time and volumetric derivative, respectively; $\alpha_{\text {cap }}$ : angle formed by the phase II and phase III limbs of the expiratory time capnogram.

The phase I is a horizontal line having a value around $0 \mathrm{mmHg}$. Phase I starts with the expiration and shows the concentration of $\mathrm{CO}_{2}$ of the gas coming from the conductive airways. However, the exact starting point of the expiration cannot be determined from tCap $(25,26)$, because the gas mixture stems from the conductive airways does not contain $\mathrm{CO}_{2}$ (Fig. 1 top). In contrast, the true starting point of exhalation can be defined by vCap from the flow and the derived volume signal (Fig.1 bottom).

The shape of the phase II exhibits a sigmoid shape. The rapid rise in partial pressure of $\mathrm{CO}_{2}$ during phase II is a physiological result of the exhalation of the gas compartment residing in the transition zone between the large conductive airways and alveolar space (Fig. 1 top and bottom). With other words, the slopes of phase II of time and volumetric 
capnogram ( $S_{I I, T}$ and $S_{I I, V}$ respectively) represents the overall width of the moving airwayalveolar gas front. Therefore, $\mathrm{S}_{\mathrm{II}, \mathrm{T}}$ and $\mathrm{S}_{\mathrm{II}, \mathrm{V}}$ are a summation result of simultaneous convective and diffusive processes in the airways leading to the mixture of alveolar and conductive dead space gas compartments (27-30). The slower the expiratory flow, the higher the role of diffusion is in determining $\mathrm{S}_{\mathrm{II}, \mathrm{T}}$ and $\mathrm{S}_{\mathrm{II}, \mathrm{V}}$. As a consequence of the exponentially decelerating expiratory flow, the $\mathrm{S}_{\mathrm{II}, \mathrm{T}}$ is normally steeper than $\mathrm{S}_{\mathrm{II}, \mathrm{V}}$ (Fig. 1 top and bottom).

Phase III shows a plateau with a normal slope of $2-3 \mathrm{mmHg} / \mathrm{s}(0.27-0.4 \mathrm{kPa} / \mathrm{s})$. It corresponds to the concentration of $\mathrm{CO}_{2}$ of the mixed gas emptying from the alveolar space. The mild slopes of phase III of the time and volumetric capnogram ( $\mathrm{S}_{\text {III,T }}$ and $\mathrm{S}_{\text {III,V }}$ respectively) of normal $S_{\text {III }}$ are determined by the physiological fact that ventilation is periodic, while perfusion is a continuous process, i.e. the $\mathrm{CO}_{2}$ is excreted in a steady state manner into a closed or even decreasing alveolar volume during expiration (28,31-33). In addition, the slopes of phase III are affected by similar diffusive and convective processes like phase II. Since velocity of the expiratory flow decreases during expiration, phases I and II are generally relatively longer, and phase III is relative shorter in vCap compared to those of tCap curve. Consequently, the $S_{\text {III,V }}$ of vCap is somewhat steeper (18-22,24,27,31,34-38) than $S_{\text {III,T. }}$

The transition indices $\left(\alpha_{c a p}, D_{2 \min }\right.$ and $\left.D_{2 V \min }\right)$ reside in the same part of the capnogram. The $\alpha_{\text {cap }}$ is composed of phases II and III and ranges $100-110^{\circ}$ at standard monitoring speed $(12.5 \mathrm{~mm} / \mathrm{s}) . \mathrm{D}_{2 \min }$ and $\mathrm{D}_{2 \mathrm{Vmin}}$ represent a curvature with variable but relatively small radius at the virtual intersection point of phases II and III (39). The meanings of the transition indices are different. $\alpha_{\text {cap }}$ reflects the relationship between the overall gas front and the alveolar gas volume, while $\mathrm{D}_{2 \min }$ and $\mathrm{D}_{2 \mathrm{Vmin}}$ are related to the internal surface of the moving $\mathrm{CO}_{2}$ diffusion front in the airways during expiration.

The peak value of phase III is the partial pressure of end-tidal $\mathrm{CO}_{2}\left(\mathrm{P}_{\mathrm{ETCO}_{2}}\right) \cdot \mathrm{P}_{\mathrm{ETCO}_{2}}$ ranges from 30 to $43 \mathrm{mmHg}(4.0-5.7 \mathrm{kPa}, 4-5.6 \mathrm{vol} \%)$ which is $2-3 \mathrm{mmHg}$ lower than the $\mathrm{PaCO}_{2}$.

Dead space parameters can also be derived from volumetric capnogram (Fig. 1 bottom). Fowler dead space $\left(\mathrm{V}_{\mathrm{DF}}\right)$ represents the anatomic dead space volume of the conducting airways. The amount of $\mathrm{V}_{\mathrm{DF}}$ is $\sim 2 \mathrm{ml} / \mathrm{kg}(\sim 150 \mathrm{ml})$, i.e., $25-33 \%$ of tidal volume 
$\left(\mathrm{V}_{\mathrm{T}}\right)$. The physiological dead space includes also the ventilated but not perfused alveolar volume, and leads to wasted ventilation. It can be estimated by using the Bohr's method, and is referred to as physiological or Bohr dead space $\left(V_{D B}\right)(40,41)$. $V_{D B}$ normally exceeds the value of $\mathrm{V}_{\mathrm{DF}}(\sim 2.2 \mathrm{ml} / \mathrm{kg})$. Consequently, the volume of ventilated but not perfused alveoli can be estimated as 2.5-3.3\% of $\mathrm{V}_{\mathrm{T}}$. The dead space according to Enghoff's modification, the Enghoff dead space $\left(\mathrm{V}_{\mathrm{DE}}\right)$ takes also into account the whole ventilation perfusion mismatch, i.e., the amount of not ventilated but still perfused alveoli (42). This "virtual, missing part" of the $\mathrm{V}_{\mathrm{T}}$ with decreased but retained fraction of pulmonary circulation corresponds to the intrapulmonary shunt, increasing normally with age, and/or supine position. The difference between the Enghoff and Bohr dead space parameters ( $\left.V_{D E}-V_{D B}\right)$ can also be calculated. This difference may theoretically indicate the amount of intrapulmonary shunt separately from other type of ventilation perfusion mismatch.

Under pathophysiological conditions, ventilation and/or perfusion heterogeneities develop in time and space, which promotes lung micro- and/or macro-compartments with various $\mathrm{CO}_{2}$ concentrations. The expiratory flow pattern alters in the large and small airways and even in the acini. The concentration of excreted $\mathrm{CO}_{2}$ into the alveolar gas and its transport can also be affected by pathological processes $(14,15,33,43)$. The $S_{\text {II }}$ of the capnogram can be concerned by opposing effects. The heterogeneous start of lung emptying, the reduced airway lumen and increased tissue damping may all contribute to its decreases, whereas an elevated elastic recoil and a low alveolar $\mathrm{CO}_{2}$ content may counteract these changes in $S_{\text {III }}$ The $S_{\text {III }}$ of the capnogram is considered to reflect the summation of the ventilation heterogeneities relating to the working alveolar compartments with different time constants and the ventilation-perfusion mismatch as concerns the dead space and/or intrapulmonary shunt. Expiratory flow alterations can modify the transition parameters $\left(\alpha_{\text {cap }}\right.$, $\mathrm{D}_{2 \text { min }}$ and $\mathrm{D}_{2 \text { Vmin }}$ ) between phase II and III. The difference between $\mathrm{P}_{\mathrm{ETCO}_{2}}$ and $\mathrm{PaCO}_{2}$ increases in case of non-sequential dead space (i.e. pulmonary embolism). Conversely, any pathological process with serial and/or parallel dead space leads to elevation in $\mathrm{S}_{\mathrm{III}}$ and subsequent reduction in $\mathrm{P}_{\mathrm{ETCO}}-\mathrm{PaCO}_{2}$ difference in time capnogram. Therefore, the chronic divergence can only be evaluated with volumetric capnography similar to the dead spaces. The absolute and/or relative increase of $\mathrm{V}_{\mathrm{DF}}$ will elevate the absolute and/or relative ineffective part of ventilation. Increase in $\mathrm{V}_{\mathrm{DB}}$ may occur during overinflation of the lung, or in right ventricular failure, hypovolaemia, and low cardiac output. $\mathrm{V}_{\mathrm{DE}}$, or especially $\mathrm{V}_{\mathrm{DB}}-$ 
$\mathrm{V}_{\mathrm{DE}}$ increases typically during perioperative period resulting from different restrictive pulmonary pathologies serving as a most frequent cause of postoperative hypoxaemia.

\subsection{Clinical aspects}

In the clinical practice, capnography, usually the tCap is the most reliable method to position endotracheal tube or any supraglottic airway device fast and correctly (44). Monitoring of exhaled $\mathrm{CO}_{2}$ help the team recognize over-insertion of endotracheal tube into the right main stem bronchus, ventilation circuit disconnection, leakage, or malfunction of flow control valves of breathing circuit (14). The $\mathrm{PETCO}_{2}$ value is generally considered as a valuable monitoring tool to recognize absolute or relative minute volume abnormalities, metabolic disturbance, pulmonary embolism $(27,45,46)$, or to wean the patient from the respirator (47). Capnography gives more direct information about changes in ventilation and/or perfusion than pulse oximetry $(4,48,49)$, due to various "bedside physiological" facts. Capnography detects gas exchange alterations more proximally than pulse oximeter, variations in oxygen-saturation is blunted by the sigmoid shape of oxygen-haemoglobin dissociation curve, $\mathrm{CO}_{2}$ has higher diffusion ability than $\mathrm{O}_{2}$, and a large gas diffusion gradient at high $\mathrm{FiO}_{2}$. (In case of $\mathrm{CO}_{2}$ it can be built by the patient.) The vCap technique displays the $\mathrm{V}^{\prime} \mathrm{CO}_{2}$ to elucidate metabolic status, and based on $\mathrm{V}_{\mathrm{DF}}$, the ineffective ventilation to optimize $\mathrm{V}_{\mathrm{T}}$ and respiratory frequency. Supplemental monitoring of a ventilated patient with rough qualitative bedside estimation of the $S_{\text {III }}$ to assess alveolar emptying can also support clinical decision making (19,21,33,50-53). Consequently, international recommendations for standards nowadays require the monitoring of ventilation with capnography in all patients undergoing sedation or general anaesthesia to confirm correct placement of endotracheal tube and to identify abnormalities of minute ventilation $(54,55)$.

Nevertheless, $\mathrm{CO}_{2}$ is an endogenous indicator during expiration. Consequently, the capnogram - with arterial blood gas - has a strong potential to reveal complex bedside information about the whole expiratory course and pulmonary microcirculation. Therefore this technique can be used for a lot more than to verify the technical correctness of the airway management and respiratory therapy as a polar question. Capnography also provides important bedside pathophysiological information about the uniformity of lung emptying and adverse changes in the overall airway geometry $(19,21,33,50,52,53)$, and it can serve as a valuable tool for the recognition of pulmonary microcirculatory abnormalities $(27,45,46)$. 
Nonetheless, from pathological point of view, capnography nowadays can be considered as the "most frequently monitored, less frequently evaluated bedside modality" in the clinical practice. Characterization of the relationships between standard lung function parameters and capnographic indices may facilitate an understanding of the various shapes of the capnogram $(52,53,56)$. However, the earlier studies demonstrating associations between the capnographic slope factors with the forced expiratory volume in $1 \mathrm{~s}\left(\mathrm{FEV}_{1}\right)$ $(53,56)$ and the peak expiratory flow $(52,56)$ were limited to spontaneously breathing subjects. Despite the particular importance of recognizing adverse alterations in the pulmonary system in mechanically ventilated patients, and the obvious importance of respiratory tissue elastance in determining the expiratory flow and the rate of $\mathrm{CO}_{2}$ clearance, details as to how the resistive and/or elastic properties of the pulmonary system affect the various indices derived from the capnogram are essentially lacking from the literature.

Moreover, the sensitivity of $\mathrm{S}_{\mathrm{III}}$ to ventilation and ventilation/perfusion abnormalities suggested its clinical usefulness for the detection of respiratory abnormalities or the subsequent ventilatory and/or pharmacological interventions. Numerous studies have demonstrated that the magnitude of $S_{\text {III }}$ reflects the severity of emphysema or asthma $(15,33,36,43,52,53,57)$, cystic fibrosis and bronchiectasis (38), COPD (18), chronic bronchitis $(32)$ and acute lung injury $(22,58)$. Inconsistent associations have been reported in previous attempts to clarify the quantitative relationships between capnographic and lung function indices. Earlier studies reported a strong correlation between $\mathrm{FEV}_{1}$ and $\mathrm{S}_{\mathrm{III}}(52)$ merely a modest association $(43,53)$ or even a lack of correspondence (57). Furthermore, significant correlations were observed between the total respiratory resistance (Rrs) and $\mathrm{S}_{\mathrm{III}}$ in mechanically ventilated patients, however $S_{\text {III }}$ had limited clinical applicability to predict Rrs (59). Thus, in consequence of the complex mechanisms affecting $S_{\text {III }}$, its diagnostic and/or monitoring value is far from being clear. The diverse emptying of different lung compartments with various $\mathrm{CO}_{2}$ levels is determined not only by the airway geometry, but also by the driving pressure governed by the Crs, including the chest wall and the lung. Despite the obvious importance of respiratory tissue elastance in determining the expiratory flow and the rate of $\mathrm{CO}_{2}$ clearance, the role of the respiratory elastic recoil on the capnogram shape has not been examined to date. 


\subsection{Aims}

The goals of the present thesis were to set out various investigations in large cohorts of ventilated patients with normal and diseased lungs during cardiac surgery. Studies focused particularly on the establishment of the connections between the various phase, shape, dead space or pulmonary shunt circulation parameters of the time or volumetric capnogram and those reflecting the airway and lung tissue mechanics, expiratory flow and gas exchange. These measurements were designed to be performed under baseline condition after induction of anaesthesia, and after a complex challenge dominated by elevation of airway resistance performed by cardiopulmonary bypass (CPB).

As a further goal, we also systematically investigated whether $S_{\text {III }}$ is affected by changes in both airway caliber and the Crs in mechanically ventilated patients. We attempted to clarify the contribution of the altered airway properties and tissue mechanics at baseline after induction of anaesthesia and after challenge with increasing PEEP. These measurements were evaluated to assess whether homogeneity or heterogeneity of lung parenchyma indicated by the $S_{\text {III }}$ provides suitable information to conclude on the gas exchange as the primary function of lung. We also elucidated whether the reliability of $S_{\text {III }}$ depends from the working lung volume, consequently it has to be used only with other parameters referring to lung volume.

\section{METHODS}

\subsection{Patients}

The subjects of both studies presented in this thesis were patients scheduled for elective open heart surgery. The protocols were approved by the Human Research Ethics Committee of Szeged University, Hungary (no. WHO 2788). All the patients received appropriate information about the study protocol and gave their written consent to their participation.

Study 1: Capnographic parameters: correspondence with airway and tissue mechanics

One hundred and one patients (71 males, 30 females) $62 \pm 9$ (mean \pm SD) yrs. of age (range: 30-88 yrs.) undergoing elective cardiac surgery were involved in a prospective, consecutive cross sectional manner. Patients were excluded in the event of severe cardiopulmonary disorders (pleural effusion $>300 \mathrm{ml}$, ejection fraction $<30 \%$, BMI $>35 \mathrm{~kg} / \mathrm{m}^{2}$ or 
intraoperative acute asthma exacerbation). The heart surgery was necessitated by aortic $(n=70)$ and/or mitral $(n=27)$ valve disease combined with ischemic heart disease $(n=28)$, and/or other types of cardiac malformation $(n=6)$, myxoma or aneurysm of the ascending aorta. Based on earlier medical reports, the patients exhibited wide-ranging variations in pulmonary status: some had no pulmonary symptoms (i.e. no history of lung disease, a normal BMI, no pleural effusion, no pulmonary congestion, no smoking history, no wheezing periods within the past 6 months, and no history of the use of bronchodilator drugs; $\mathrm{n}=16$ ), whereas others had lung abnormalities causing restrictive (pulmonary congestion $(n=56)$ and/or obesity $(B M I \geq 31)(n=31))$ and/or obstructive changes (emphysema $(n=28)$, asthma $(n=8)$ or chronic bronchitis $(n=20))$.

Study 2: Respiratory mechanics and the capnogram phases: importance of dynamic compliance

One hundred and forty-four patients (93 males, 51 females) $62 \pm 9$ (mean \pm SD) yrs. of age (range 39-84 yrs.) undergoing elective coronary bypass surgery were examined in the supine position before the surgical procedure. The patients had various cardiac diseases including ischemic heart disease $(n=108)$, a mitral insufficiency $(n=21)$, aortic stenosis $(n=38)$, and other types of cardiac malformation $(n=5)$, such as myxoma, congenital heart disease or aortic aneurysm. The patients exhibited wide variations in their pulmonary status, with some of them having no pulmonary symptoms (i.e. no history of lung disease, normal BMI, no pleural effusion, no pulmonary congestion, no smoking history, lack of wheezing periods within the past 6 months, lack of use of bronchodilator drugs; $n=29)$. Others had lung abnormalities causing restrictive (pulmonary congestion $(n=45)$ or obesity $(B M I \geq 31 ; n=48)$ ) and/or obstructive changes (emphysema $(n=55)$, asthma $(n=14)$, chronic bronchitis $(n=25)$ or sarcoidosis $(n=1))$.

\subsection{Anaesthesia and surgery}

In both studies, the patients were praemedicated with intramuscular morphine $(0.07 \mathrm{mg} / \mathrm{kg})$ and midazolam $(0.07 \mathrm{mg} / \mathrm{kg}) 1 \mathrm{~h}$ before the operation. Anaesthesia was induced with intravenous (IV) midazolam (30 $\mu \mathrm{g} / \mathrm{kg})$, sufentanil $(0.4-0.5 \mu \mathrm{g} / \mathrm{kg})$ and propofol $(0.3-0.5$ $\mathrm{mg} / \mathrm{kg})$. Muscle paralysis was achieved with an IV bolus of rocuronium $(0.6 \mathrm{mg} / \mathrm{kg})$. The anaesthesia and muscle relaxation were maintained with IV infusions of propofol (50 
$\mu \mathrm{g} / \mathrm{kg} / \mathrm{min}$ ) and IV boluses of rocuronium $(0.2 \mathrm{mg} / \mathrm{kg}$ every $30 \mathrm{~min})$. The trachea was intubated with a cuffed endotracheal tube with an internal diameter of 7, 8 or $9 \mathrm{~mm}$, and the patients were ventilated with a Dräger Zeus anaesthesia machine (Lübeck, Germany) in volume-controlled mode with descending flow. The ventilator frequency was set to 12-14 breaths $/ \mathrm{min}$, with a tidal volume of $7 \mathrm{ml} / \mathrm{kg}$. The fraction of inspired oxygen $\left(\mathrm{FiO}_{2}\right)$ was maintained at 0.5 throughout the entire study period. Arterial blood gas samples were analysed to calculate the Horowitz coefficient $\left(\mathrm{HQ}=\mathrm{PaO}_{2} / \mathrm{FiO}_{2}\right)$.

Study 1: Capnographic parameters: correspondence with airway and tissue mechanics

During intermittent positive pressure ventilation (IPPV) a positive end-expiratory pressure (PEEP) of $4 \mathrm{cmH}_{2} \mathrm{O}$ was applied. Prior to cardiopulmonary bypass (CPB), $1500 \mathrm{ml}$ of lactated Ringer's solution was used to prime the membrane oxygenator and the tube set. Heparin (300 IU/kg) was administered with the activated anticoagulation time maintained above $400 \mathrm{~s}$. At the beginning of the $\mathrm{CPB}$, mild hypothermia was generally applied to maintain oesophageal temperature of $32{ }^{\circ} \mathrm{C}$. During cardioplegic cardiac arrest, the lung ventilation was stopped, the ventilator was disconnected and no positive airway pressure was maintained in the lungs. The lungs were then inflated 3-5 times to a peak airway pressure of $30 \mathrm{cmH}_{2} \mathrm{O}$ before declamping of the aorta in order to facilitate the removal of gas emboli from the heart and to perform lung recruitment. A similar manoeuvre was performed before ventilation restoration and weaning of the patient from the CPB. Arterial and central venous blood gas samples were analysed to calculate the Horowitz coefficient and the intrapulmonary shunt based on Fick-principle and on dead spaces.

Study 2: Respiratory mechanics and the capnogram phases: importance of dynamic compliance

To establish whether the elastic properties of the respiratory system affected the capnogram shape, the patients with respiratory symptoms were allocated into three groups, on the basis of their dynamic respiratory compliance (Crs). This Crs was determined 10 min after anaesthesia induction and a lung volume homogenization manoeuvre (i.e. lung inflation and maintenance at a trans respiratory pressure of $30 \mathrm{cmH}_{2} \mathrm{O}$ for $5 \mathrm{~s}$ ) when stable hemodynamic and ventilatory conditions at PEEP $3 \mathrm{cmH}_{2} \mathrm{O}$ have been reached (i.e. prior to the first capnogram recording). Group LC comprised patients with Crs in the lowest tenth percentile 
( $\left.\mathrm{Crs}<34.5 \mathrm{ml} / \mathrm{cmH}_{2} \mathrm{O} ; \mathrm{n}=15\right)$, and Group MC patients with $\mathrm{Crs}$ between the tenth and the ninetieth percentile $\left(34.5<\mathrm{Crs}<69 \mathrm{ml} / \mathrm{cmH}_{2} \mathrm{O} ; \mathrm{n}=85\right)$, and Group $\mathrm{HC}$ patients with Crs above the ninetieth percentile $\left(\mathrm{Crs}>69 \mathrm{ml} / \mathrm{cmH}_{2} \mathrm{O} ; \mathrm{n}=15\right)$. Patients with healthy lungs were regarded as an independent group (Group HL; $n=29$ ). The patients were classified based on the Crs measured after a lung volume recruitment manoeuvre. The characteristics of the patients in each protocol group are summarized on Table 1. The age of the patients did not differ significantly in the different groups $(\mathrm{p}=0.16)$. The ejection fraction $(\mathrm{EF})$ data were collected from preoperative echocardiography. The body mass index (BMI) of each patient was calculated.

\begin{tabular}{|c|c|c|c|c|}
\hline & $\begin{array}{l}\text { Gender* } \\
(\mathbf{m} / \mathbf{f})\end{array}$ & $\begin{array}{l}\text { Obesity* } \\
\text { (n/ow/ob) }\end{array}$ & $\begin{array}{l}\text { Pulmonary status } \\
\mathrm{E} * / \mathrm{A} * / \mathrm{CB} * / \mathrm{OLD}\end{array}$ & $\begin{array}{c}\text { Cardiac disease } \\
\text { CAD/AS/MI/LEF*/OCD }\end{array}$ \\
\hline $\begin{array}{l}\text { Group HL } \\
(\mathbf{n}=29)\end{array}$ & $20 / 9$ & $10 / 19 /-$ & $-/-/-/-$ & $26 / 3 / 3 /-/ 1$ \\
\hline $\begin{array}{c}\text { Group HC } \\
(n=15)\end{array}$ & $15 /-$ & 8/7/- & $14 /-/ 6 /-$ & $11 / 4 /-/-/-$ \\
\hline $\begin{array}{l}\text { Group MC } \\
\qquad(n=84)\end{array}$ & $56 / 28$ & $12 / 28 / 44$ & $37 / 10 / 15 / 2$ & $61 / 28 / 8 / 10 / 3$ \\
\hline $\begin{array}{l}\text { Group LC } \\
(\mathbf{n}=15)\end{array}$ & $3 / 12$ & $-/ 5 / 10$ & $4 / 4 / 4 /-$ & $10 / 3 / 1 / 8 / 1$ \\
\hline
\end{tabular}

Table 1. Number of patients with different conditions/diagnoses in each protocol group. Obesity categories: n: normal $(20 \leq \mathrm{BMI}<24)$, ow: overweight $(25 \leq \mathrm{BMI}<30)$, ob: obese: BMI>31. Pulmonary status: E: emphysema, A: bronchial asthma, $\mathrm{CB}$ : chronic bronchitis, OLD: other lung disease. Cardiac diseases: CAD: coronary artery disease, AS: aortic stenosis, MI: mitral insufficiency, LEF: low ejection fraction (EF<50\%), OCD: other cardiac disease. Pulmonary and cardiac conditions are based on previous clinical diagnoses. *: $\mathrm{p}<0.05$ between the expected and the observed frequencies in the protocol groups for each variable.

\subsection{Forced oscillatory measurements}

Study 1: Capnographic parameters: correspondence with airway and tissue mechanics

Airway and tissue mechanical properties were assessed by measuring the low-frequency forced oscillatory input impedance of the pulmonary system (ZL) (60). The common side of a T-piece was attached to a distal ET tube. The other sides of the T-piece containing two collapsible segments were connected to the respirator and the forced oscillatory 
measurement apparatus. Before the oscillatory measurements, the lungs were inflated to a pressure of approximately $30 \mathrm{cmH}_{2} \mathrm{O}$ to standardize the volume history. During short (15-s) apnoeic periods, this equipment allowed switching the patient from the respirator to the forced oscillatory system while pseudorandom pressure excitations were generated into the trachea. The pressure forcing signal contained 15 integer-multiple components in the frequency range 0.4-6 Hz. ZL was computed from the power spectra of the airway opening pressure (Pao) and tracheal airflow (V'). Pao was measured with a pressure transducer (ICS model 33NA002D; ICSensors, Milpitas, CA, USA), and V' was measured with a 28-mm ID screen pneumotachograph connected to the identical pressure transducer. A well-validated 4-parameter (61) model containing a frequency-independent airway resistance (Raw) and inertance (Iaw) and a constant-phase tissue compartment characterized by the coefficients of damping $(\mathrm{G})$ and elastance $(\mathrm{H})$ was fitted to the mean ZL data by minimizing the weighted differences between the measured and modelled impedance values:

$$
\mathrm{ZL}=\mathrm{Raw}+\mathrm{j} \omega \mathrm{Iaw}+(\mathrm{G}-\mathrm{jH}) / \omega^{\alpha}
$$

where $\omega$ is the angular frequency $(2 \pi \mathrm{f})$ and $\alpha=2 / \pi \cdot \arctan (H / G)$. The tissue resistive component (Rti) at the ventilation frequency $(0.2 \mathrm{~Hz})$ was calculated from the parenchymal damping coefficient $\left(\mathrm{Rti}=\mathrm{G} / \omega^{\alpha}\right)$. The total lung resistance $(\mathrm{RL})$ was determined as the sum of the airway resistance (Raw) and the Rti $\left(\mathrm{RL}=\mathrm{Raw}+\mathrm{G} / \omega^{\alpha}\right)$.

Study 2: Respiratory mechanics and the capnogram phases: importance of dynamic compliance

The input impedance of the respiratory system ( $\mathrm{Zrs})$ was measured, and the forcing signal contained 30 integer-multiple components of the $0.2-\mathrm{Hz}$ fundamental frequency, in the frequency range $0.2-6 \mathrm{~Hz}$.

\subsection{Recording and analyses of the expiratory capnogram}

Study 1: Capnographic parameters: correspondence with airway and tissue mechanics

A mainstream capnograph (Novametrix, Capnogard ${ }^{\circledR}$, Andover, MA, USA) and another central airflow meter (Piston Ltd., Budapest, Hungary) were connected into the ventilatory circuit at the Y-piece, and 15-s $\mathrm{CO}_{2}$ and ventilator flow traces were recorded simultaneously. 
The $\mathrm{CO}_{2}$ and ventilator flow traces were digitized and imported into custom-made signal analysis software. The slopes of phase III of the capnogram in the time $\left(\mathrm{S}_{\mathrm{III}, \mathrm{T}}\right)$ and in the volumetric $\left(\mathrm{S}_{\text {III,V) }}\right)$ domains were determined by fitting a linear regression line to the last twothirds of each phase-III traces (Fig. 1) $(43,58)$. The phase-II slopes of the time $\left(\mathrm{S}_{\mathrm{II}, \mathrm{T}}\right)$ and volumetric $\left(\mathrm{S}_{\mathrm{II}, \mathrm{V}}\right)$ capnograms were determined by calculating the slopes of the best-fitting line around the inflection point $( \pm 20 \%)(62)$. Each slope was divided by the average corresponding $\mathrm{CO}_{2}$ concentration in the mixed expired gas to obtain normalized time $\left(\mathrm{Sn}_{\mathrm{II}, \mathrm{T}}\right.$ and $\left.\mathrm{Sn}_{\text {III,T }}\right)$ and volumetric $\left(\mathrm{Sn}_{\text {II,V }}\right.$ and $\mathrm{Sn}_{\text {III,V) }}$ phase II and III slopes $(27,63,64)$. This normalization was made only for the slope indices, as performed earlier before and after CPB $(27,63,64)$. The angle $\left(\alpha_{c a p}\right)$ formed by the phase II and III limbs of the expiratory time capnogram was also calculated by using a standard monitoring speed of $12.5 \mathrm{mmHg} / \mathrm{s}$. The transition rates of change from phase II to phase III in the time $\left(D_{2 m i n}\right)$ and volumetric $\left(\mathrm{D}_{2 \mathrm{Vmin}}\right)$ capnograms reflecting the curvature were calculated as the minima of the secondorder time and volumetric derivatives (65).

Besides these shape factors, dead space parameters were derived from the volumetric capnogram. The Fowler dead space $\left(\mathrm{V}_{\mathrm{DF}}\right)$ was determined by calculating the expired gas volume until the inflection point of phase II was reached in the volumetric capnogram $(66,67)$. The physiological dead space was assessed by the Bohr method (40) and referred as Bohr dead space $\left(\mathrm{V}_{\mathrm{DB}}\right)(41)$ :

$\mathrm{V}_{\mathrm{DB}}=\left(\mathrm{P}_{\mathrm{ACO}_{2}}-\mathrm{P}_{\overline{\mathrm{ECO}}}\right) / \mathrm{P}_{\mathrm{ACO}_{2}}$

where $\mathrm{P}_{\mathrm{ACO}_{2}}$ is the mean alveolar $\mathrm{CO}_{2}$ concentration located at the midpoint of the phase III in the expired $\mathrm{CO}_{2}$ curve, and $\mathrm{P}_{\overline{\mathrm{ECO}}}$ is the mixed partial pressure of $\mathrm{CO}_{2}$ during the entire expiration (68). The latter is calculated as the ratio of the tidal elimination of $\mathrm{V}^{\prime} \mathrm{CO}_{2}$ obtained by integrating the flow and $\mathrm{CO}_{2}$ signals over the entire breath and the tidal volume (Fig. 1 bottom). The dead space according to Enghoff's modification $\left(\mathrm{V}_{\mathrm{DE}}\right)$ was calculated (42) as:

$\mathrm{V} \mathrm{DE}=\left(\mathrm{PaCO}_{2}-\mathrm{P}_{\overline{\mathrm{E}} \mathrm{CO}_{2}}\right) / \mathrm{PaCO}_{2}$

where $\mathrm{PaCO}_{2}$ is the partial pressure of $\mathrm{CO}_{2}$ in the arterial blood (Fig. 1 bottom). We also calculated the differences between the Enghoff and Bohr dead space parameters $\left(V_{D E}-V_{D B}\right)$ 
representing the pulmonary shunt circulation. The intrapulmonary shunt blood flow (Qs/Qt) was additionally assessed via the Fick equation (Fig. 1 bottom).

Study 2: Respiratory mechanics and the capnogram phases: importance of dynamic compliance

Changes in partial $\mathrm{CO}_{2}$ pressure in the exhaled gas during mechanical ventilation were measured with a calibrated side-stream capnograph (Ultima ${ }^{\mathrm{TM}}$, Datex/Instrumentarium, Helsinki, Finland). Since capnograms are displayed in clinical routine in the time domain, time capnography was applied in each patient to record $\mathrm{CO}_{2}$ changes. To minimize the possible drawback of this time domain analyses, we paid attention to involve only the linear part of the $\mathrm{CO}_{2}$ trace in the readings of $\mathrm{S}_{\mathrm{III}}$. Nevertheless, volumetric capnography may allow a better distinction between the phases $(15,18,22,27,33,34,58,63)$ and thus, in a subgroup including the last 68 patients, the flow during mechanical ventilation was simultaneously recorded with the $\mathrm{CO}_{2}$ traces by introducing an additional pneumotachograph into the ventilation circuit. This allowed the analyses of volumetric capnograms in 20, 7, 32 and 9 patients in the Groups $\mathrm{HL}, \mathrm{HC}, \mathrm{MC}$ and $\mathrm{LC}$, respectively. The $15-\mathrm{s} \mathrm{CO}_{2}$ and respiratory flow traces were imported into commercial signal analysis software (Biopac, Santa Barbara, CA, USA). Assessing the slopes of phase III of the capnogram in time $\left(\mathrm{S}_{\mathrm{III}, \mathrm{T}}\right)$ and in volumetric $\left(\mathrm{S}_{\mathrm{III}, \mathrm{V}}\right)$ domains and their normalizations were performed similar to those applied in Study 1.

In both studies, and at every experimental condition, 3 to 5 expiratory traces in each recording were analysed, resulting in the ensemble-averaging of 10-12 values for further analysis in each patient.

\subsection{Analysis of the expiratory flow}

Study 1: Capnographic parameters: correspondence with airway and tissue mechanics

To characterize the expiratory flow pattern, the expiratory phases of each $\mathrm{V}$ ' recordings were analysed by fitting an exponential function to the elevating limb (69):

$V^{\prime}(t)=V^{\prime} p l-P F \cdot e^{-L F \cdot t}$

where $\mathrm{V}^{\prime}$ pl is the plateau flow before the beginning of the next inspiration, $\mathrm{PF}$ is the peak expiratory flow, and LF is related to the curvature of the expiratory curve. The parameter LF 
is related to the curvature of the expiratory curve; a larger value indicates a more concave shape in the late flow. Model fitting to the serial data points from the peak flow was performed until $90 \%$ of the equilibrium value of $\mathrm{V}^{\prime}(\mathrm{t})$ was reached.

\subsection{Calculating intrapulmonary shunt based on Fick principle and classic shunt equation}

Study 1: Capnographic parameters: correspondence with airway and tissue mechanics

The ratio of the shunted cardiac output $(\mathrm{Qs})$ to total cardiac output $(\mathrm{Qt})$ is referred to classic shunt equation and can be calculated as:

$\mathrm{Qs} / \mathrm{Qt}=\mathrm{CtcO}_{2}-\mathrm{CtaO}_{2} / \mathrm{CtcO}_{2}-\mathrm{CtvO}_{2}$

where Qs is the shunted-, Qt is the total cardiac output, $\mathrm{CtcO}_{2}, \mathrm{CtaO}_{2}, \mathrm{CtvO}_{2}$, are the pulmonary capillary, arterial, and mixed venous oxygen content respectively. The pulmonary capillary oxygen content was assessed from the oxygen content equation:

$\mathrm{CtcO}_{2}=\mathrm{Hb} \times \mathrm{SaO}_{2} \times 1.34+0.0031 \times \mathrm{PaO}_{2}$

When oxygen-saturation is estimated 1 and oxygen partial pressure is expected to be equal to alveolar oxygen partial pressure that resulting from $\mathrm{FiO}_{2}$ applied at the measurement.

\subsection{Measurement protocols}

Study 1: Capnographic parameters: correspondence with airway and tissue mechanics

Two sets of measurements were made under the open-chest condition 5 min before the CPB and 5 min after the patient was weaned from the $\mathrm{CPB}$. Recruitment manoeuvres were performed before the weaning from the CPB. Each data collection period started with recordings of 3 to 5 capnogram traces. During this period, an arterial blood gas sample was taken to measure $\mathrm{PaO}_{2}$ and $\mathrm{PaCO}_{2}$ for the calculation of $\mathrm{HQ}$ and $\mathrm{VDE}$, respectively. The total lung resistance $\left(\mathrm{R}_{\mathrm{vent}}\right)$ and compliance $\left(\mathrm{C}_{\mathrm{vent}}\right)$ displayed by the respiratory monitor of the ventilator were registered at this stage of the protocol. The data collections under both conditions were supplemented by recordings of 3 to $5 \mathrm{ZL}$ data epochs at 1-min intervals. 
Study 2: Respiratory mechanics and the capnogram phases: importance of dynamic compliance

The scheme of the experimental protocol is outlined in Fig. 2.

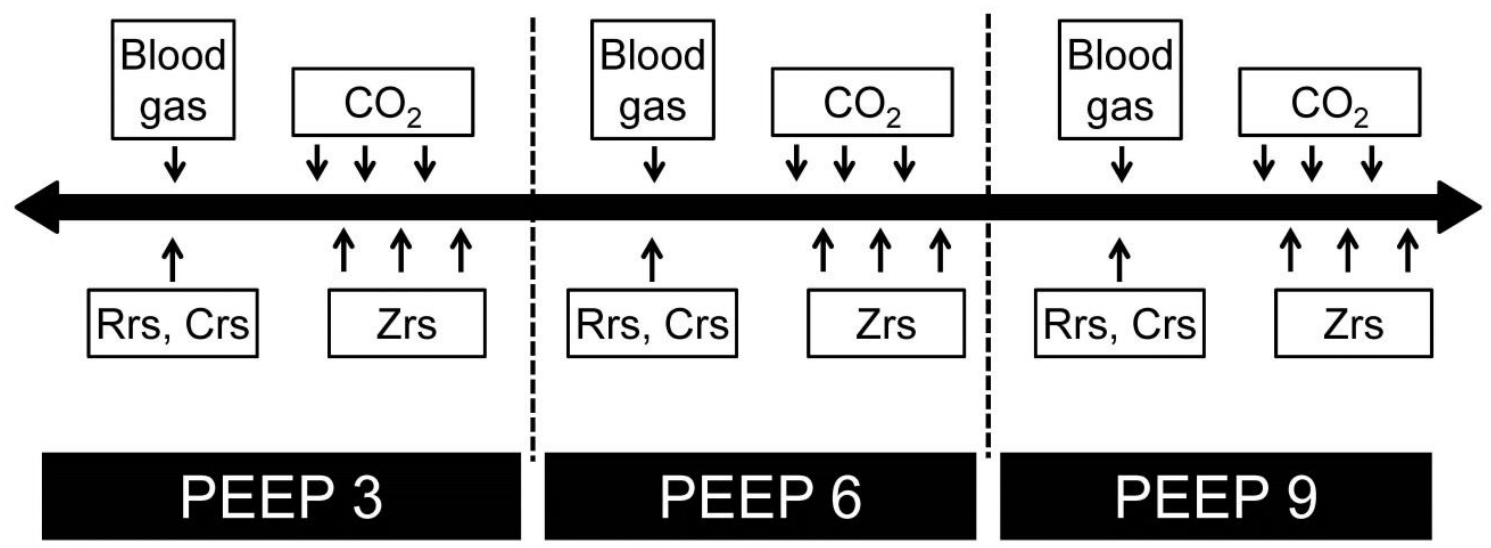

Figure 2. Timeline of the experimental protocol. Rrs and Crs: readings from the respirator display; $\mathrm{CO}_{2}$ : recording of capnogram curves; Zrs: forced oscillatory measurement of respiratory system impedance.

When stable hemodynamic and respiratory mechanical conditions had been reached while PEEP was maintained at $3 \mathrm{cmH}_{2} \mathrm{O}$, an arterial blood gas sample was taken, and dynamic compliance (Crs) was recorded from the display of the respirator. The first capnogram trace was then collected followed by recording of the first Zrs data epoch. Two more capnographic and Zrs measurements were then made in alternating sequence at 60 -s intervals. PEEP was next elevated to 6 and then $9 \mathrm{cmH}_{2} \mathrm{O}$, a 3 -min equilibration period being permitted after each step, and the data collection procedure was repeated.

\subsection{Statistical analyses}

In both studies, scatters in measured variables are expressed as SEM values. The Pearson test was applied to analyse the correlations between the different variables. Values $\mathrm{p}<0.05$ were considered to be statistically significant. All reported $\mathrm{p}$ values are two-sided. The statistical tests were performed with a SigmaPlot statistical software package (Version 12, Systat Software, Inc. Chicago, IL, US) except the Steiger's Z-test (70).

Study 1: Capnographic parameters: correspondence with airway and tissue mechanics 
The necessary sample size estimation was applied to involve sufficient number of patients for the detection of clinically relevant significances. The type 1 error rate was set to 0.05 , the statistical power was set to 0.85 and the clinically relevant effect size (alternative hypothesis) was considered to detect correlation coefficients $r=0.3$ versus $r=0$. The necessary sample size was 96 .

In the event of passing the normality test (marked in footnotes), paired t-tests were used to examine the statistical significance of the changes induced in the parameters by the CPB. Wilcoxon signed-rank tests were applied otherwise to verify the significance of the changes in the mechanical, capnographic or gas exchange parameters. The comparison of Pearson correlation coefficients was made by Steiger's Z-test; these tests were performed between the particular and the nearest $r$ values. Subgroups of patients were formed based on the initial HQ level (high and low 25 percentile), and based on the extremity of changes after surgery (top 25 percentile increase and bottom 25 percentile decrease in HQ, respectively). Time domain capnogram slope indices and Raw and $\mathrm{C}_{\mathrm{vent}}$ and their changes after the surgery were also correlated in these subgroups and were compared to the results obtained from the pooled population.

Study 2: Respiratory mechanics and the capnogram phases: importance of dynamic compliance

The normality of the data was tested with the Kolgomorov-Smirnov test with Lilliefors correction. Two-way repeated measures analysis of variance (ANOVA) with including an interaction term was used with the variables PEEP $\left(3,6\right.$ and $\left.9 \mathrm{cmH}_{2} \mathrm{O}\right)$ and the group allocation (Groups HL, LC, MC and HC) to establish the effects of lung volume and Crs on the respiratory mechanical, blood gas and capnographic variables. This statistical method was utilized to test the hypothesis that the level of Crs affects the PEEP-dependent changes in the respiratory mechanical and capnogram variables. Multiple linear regression analysis was performed to establish whether the levels of BMI and EF affect Crs. The Holm-Sidak multiple comparison procedure was adopted to compare the variables in the various study groups under different conditions. Chi-square test was used to assess whether there is a significant difference between the expected and the observed frequencies of gender, obesity, pulmonary and cardiac diseases in the protocol groups. 


\section{RESULTS}

Study 1: Capnographic parameters: correspondence with airway and tissue mechanics

Parameters reflecting the lung mechanics and the expiratory flow are demonstrated in Fig.3.
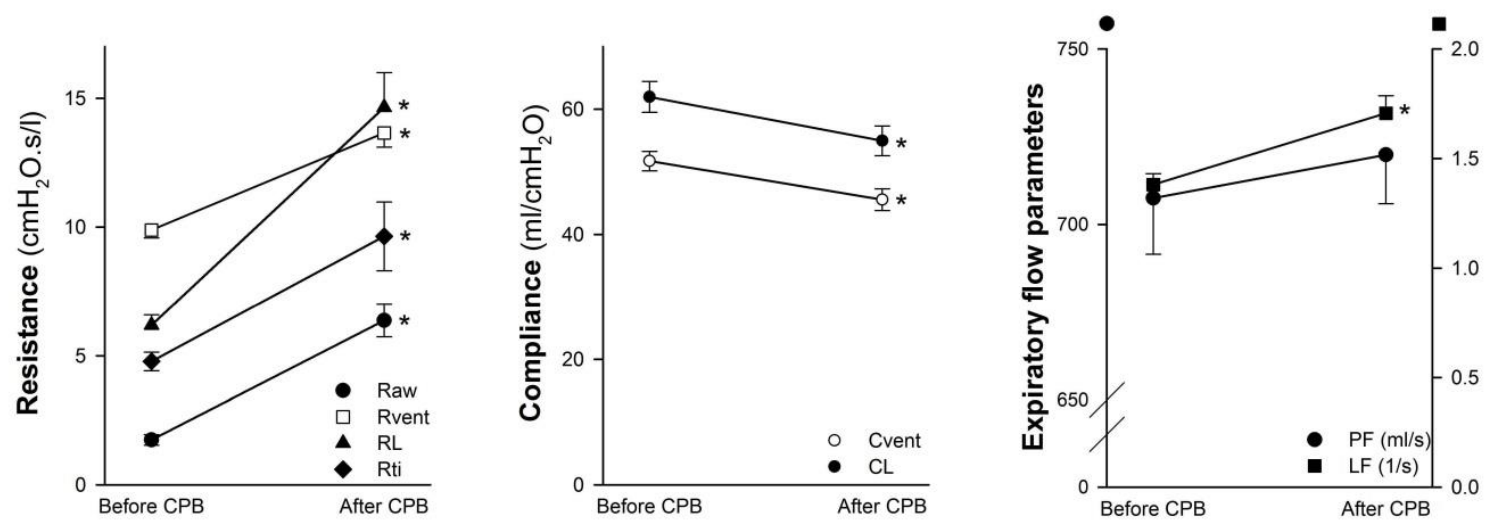

Figure 3. Resistive (Raw: airway resistance, $\mathrm{R}_{\mathrm{vent}}$ : total lung resistance displayed by the ventilator, RL: total lung resistance obtained by oscillometry, Rti: tissue resistance obtained by oscillometry) and elastic lung mechanical parameters $\left(\mathrm{C}_{\text {vent }}\right.$ : compliance displayed by the ventilator, $\mathrm{CL}$ : compliance determined by oscillometry) and expiratory flow indices (PF: peak flow, LF: late flow) before and after the cardiopulmonary bypass (CPB). *: $\mathrm{p}<0.05$ before vs. after the CPB. Values are expressed as mean \pm SEM.

All the resistive parameters, including those reflecting the flow resistance of the airways (Raw), or of the lung tissues (Rti), or the combination of these compartments ( $R_{\text {vent }}$ and RL), exhibited marked and statistically significant increases after CPB ( $<<0.0001$ for each). Conversely, more moderate, but still highly significant decreases were observed following $\mathrm{CPB}$ in the compliance parameters determined at end-expiratory lung volume by the oscillometry (CL) or at end-inspiratory lung volume by the ventilator $\left(\mathrm{C}_{\mathrm{vent}}\right)(\mathrm{p}<0.0001$ for both). CPB induced no statistically detectable changes in $\mathrm{PF}(\mathrm{p}=0.5)$, whereas the parameter LF, reflecting the curvature of the late flow, increased significantly $(p<0.0001)$. The CPBinduced adverse lung mechanical changes were also reflected in the significant decrease in HQ (from $371 \pm 11$ to $350 \pm 14 \mathrm{mmHg} ; \mathrm{p}=0.038^{1}$ ).

Fig. 4 depicts the indices derived from the time and volumetric capnographic measurements before and after the CPB. Marked and statistically significant increases were observed in the time and volumetric parameters reflecting the phase-III slope of the expired $\mathrm{CO}_{2}(\mathrm{p}<0.0001$

\footnotetext{
${ }^{1}$ Shapiro-Wilk test for normality passed $(\mathrm{p}>0.41)$
} 
for $\mathrm{S}_{\text {III,T }}, \mathrm{Sn}_{\text {III,T, }} \mathrm{S}_{\text {III,V }}$ and $\mathrm{Sn}_{\text {III,V) }}$ after the CPB. The slopes of phase II revealed significant decreases following CPB $\left(\mathrm{p}<0.0001\right.$ for both $\mathrm{S}_{\text {II,T }}$ and $\left.\mathrm{S}_{\text {IIIV }}\right)$, whereas these drops were no longer detectable after normalization to the $\mathrm{CO}_{2}$ concentration in the mixed expired gas ( $\mathrm{p}=0.4$ and 0.9 for $\mathrm{Sn}_{\mathrm{II}, \mathrm{T}}$ and $\mathrm{Sn}_{\mathrm{II}, \mathrm{V}}$, respectively ${ }^{1}$ ). CPB increased the curvature representing the transition from phase II to phase III ( $p<0.0001$ for both $\mathrm{D}_{2 \min }$ and $\mathrm{D}_{2 \mathrm{Vmin}}$ ). Uniform decreases were detected in $V_{D F}$ and $V_{D B}(p<0.0001)$ after the $C P B$, whereas $V_{D E}$ increased significantly $(\mathrm{p}<0.0001)$. These changes in the dead space parameters resulted in significant elevations in the shunt parameters reflecting the alterations in lung ventilation $(\mathrm{p}=0.02$ and $\mathrm{p}<0.0001$ for $\mathrm{V}_{\mathrm{DB}}-\mathrm{V}_{\mathrm{DF}}$ and $\mathrm{V}_{\mathrm{DE}}-\mathrm{V}_{\mathrm{DB}}$, respectively ${ }^{2}$ ) and perfusion $(\mathrm{Q} / \mathrm{Qt}, \mathrm{p}<0.0001)$.

\footnotetext{
${ }^{2}$ Shapiro-Wilk test for normality passed $(\mathrm{p}>0.13)$
} 

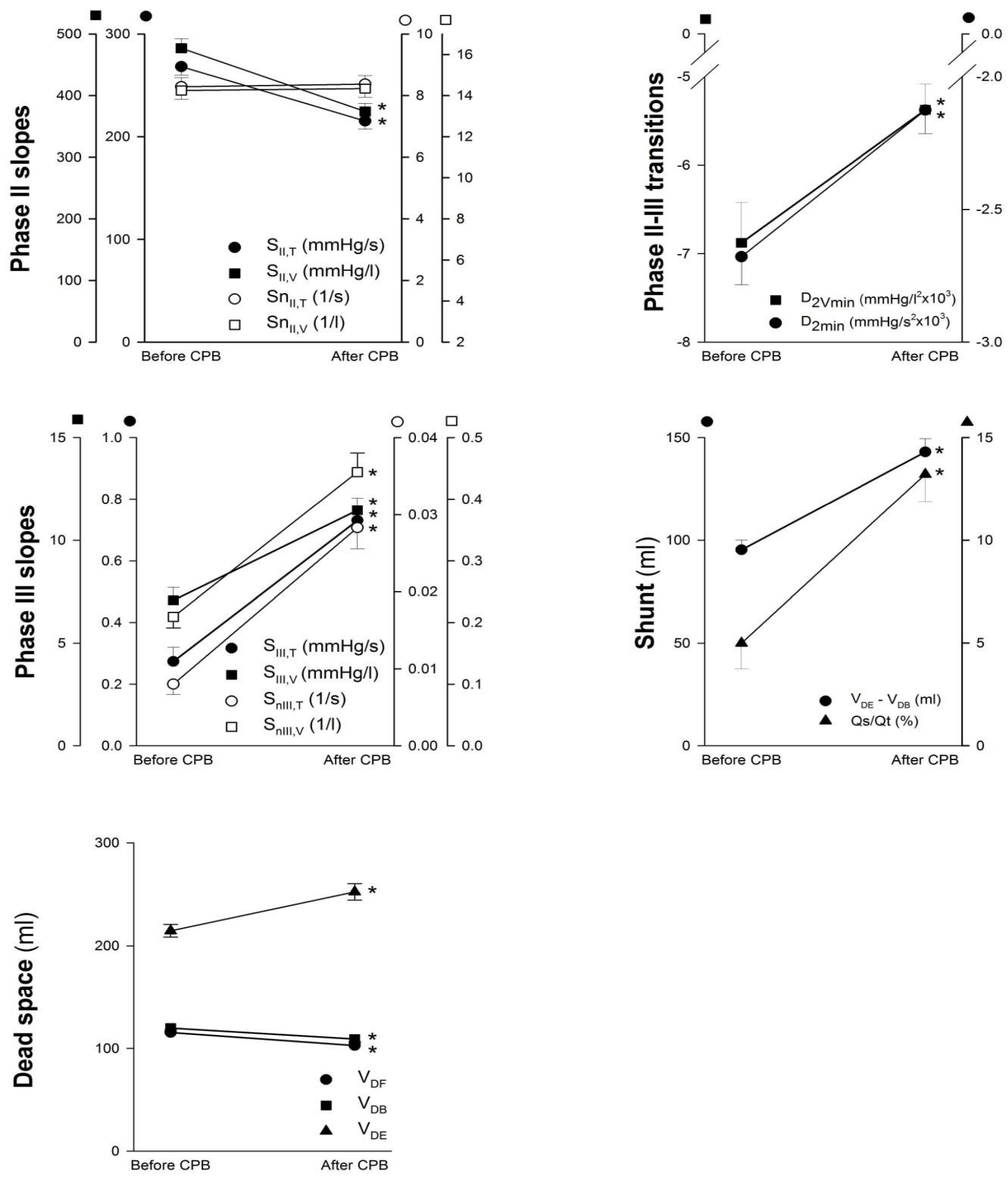

Figure 4. Indices derived from the time and volumetric capnographic measurements before and after the cardiopulmonary bypass (CPB). $\mathrm{S}_{\mathrm{II}, \mathrm{T}}, \mathrm{S}_{\mathrm{II}, \mathrm{V}}$, and $\mathrm{Sn}_{\mathrm{II}, \mathrm{T}}, \mathrm{Sn}_{\mathrm{II}, \mathrm{V}}$ are absolute and normalized phase-II slopes of the time and volumetric capnogram, respectively. $\mathrm{S}_{\text {III,T }}, \mathrm{S}_{\mathrm{III}, \mathrm{V}}$, and $\mathrm{Sn}_{\mathrm{III}, \mathrm{T}}, \mathrm{Sn}_{\mathrm{III}, \mathrm{V}}$ are absolute and normalized phaseIII slopes of the time and volumetric capnogram, respectively. $\mathrm{D}_{2 \min }$ and $\mathrm{D}_{2 \mathrm{Vmin}}$ represent the curvature at the phase II-III transitions, calculated as the minimum of the second-order time and volumetric derivative, respectively. $\mathrm{V}_{\mathrm{DF}}, \mathrm{V}_{\mathrm{DB}}$ and $\mathrm{V}_{\mathrm{DE}}$ denote dead spaces according to Fowler, Bohr and Enghoff. Qs/Qt reflects the intrapulmonary shunt. Values are expressed as mean \pm SEM. 
Fig. 5 illustrates the strengths of the correlations between the lung mechanical parameters (x-axis) and the time and volumetric capnographic parameters reflecting the slopes, transitions, dead-space and shunt fractions (y-axis).

The lung resistive parameters exhibited the closest associations with the phase-III slope capnographic parameters $(\mathrm{p}<0.0001)$, particularly after the $\mathrm{CPB}$, when all the indices reflecting the resistive properties of the pulmonary system were markedly elevated ( $<<0.0001 ;$ Fig. 5, top panels). Significant, but somewhat weaker correlations were observed between the lung resistive parameters and the ventilation dead-space parameters $V_{D F}$ $(\mathrm{p}<0.0001)$ and $\mathrm{V}_{\mathrm{DB}}(\mathrm{p}<0.0001)$. More specifically, the mechanical parameter representing the flow resistance of the airways (Raw) correlated best $(\mathrm{p}<0.0001)$ with the $\mathrm{S}_{\text {III,T }}(\mathrm{r}=0.63$ and 0.68 for $\mathrm{S}_{\text {III,T }}$ before and after the $\mathrm{CPB}$, respectively; $\left.\mathrm{p}<0.0001\right)$. Moreover, Raw correlated significantly with $\mathrm{S}_{\text {III,V }}\left(\mathrm{r}=0.43\right.$ and 0.55 for $\mathrm{S}_{\mathrm{III}, \mathrm{V}}$ before and after CPB, respectively, $\mathrm{p}<0.0001)$. Normalization of the phase-III slopes to the $\mathrm{CO}_{2}$ concentration in the mixed expired gas did not affect these relationships noticeably $(\mathrm{p}=0.71)$. Conversely, the mechanical parameters characterizing lung tissue elasticity $\left(\mathrm{H}\right.$ and $\left.\mathrm{C}_{\mathrm{vent}}\right)$ showed the closest ( $\mathrm{p}=0.006)$ relationships with the time capnographic parameters describing the phase II ( $r=0.65$ and 0.41 between $\mathrm{H}$ and $\mathrm{S}_{\mathrm{II}, \mathrm{T}}$ before and after the CPB, respectively; $\mathrm{p}<0.0001$ ). The pulmonary elastance and compliance parameters also revealed close associations with the capnographic indices reflecting the curvatures of the transitions between the phases, particularly before the CPB ( $r=-0.57$ between $\mathrm{H}$ and $\left.\mathrm{D}_{2 \min } ; \mathrm{p}<0.0001\right)$. The early and latephase expiratory flow parameters revealed strong associations between PF and the deadspace indices both before and after the CPB. LF exhibited the strongest correlation with $\mathrm{Sn}_{\text {III,V }}(\mathrm{r}=0.53 ; \mathrm{p}<0.0001)$. 
Before CPB

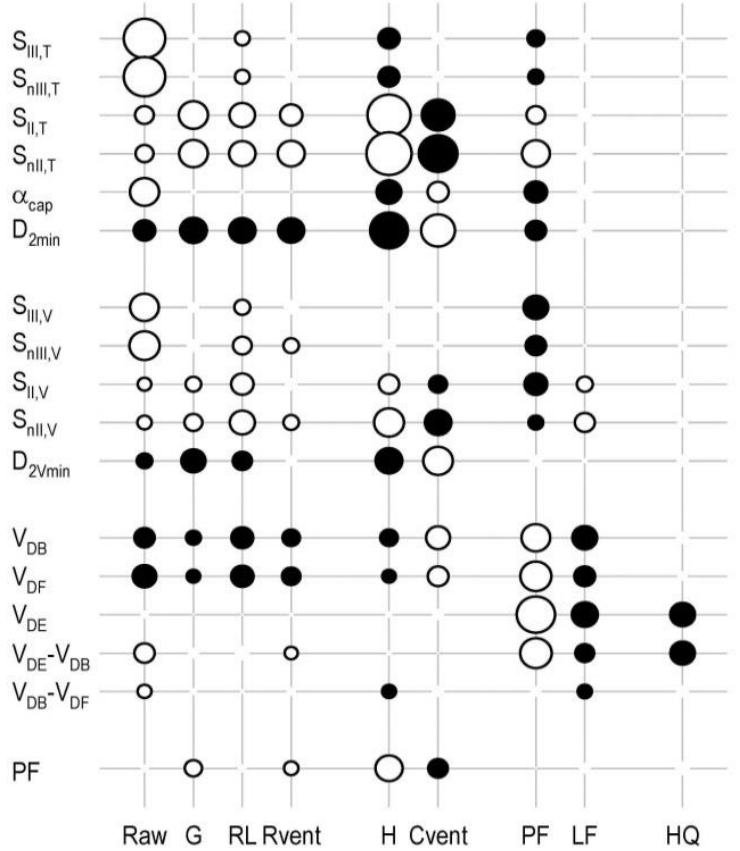

\section{After CPB}

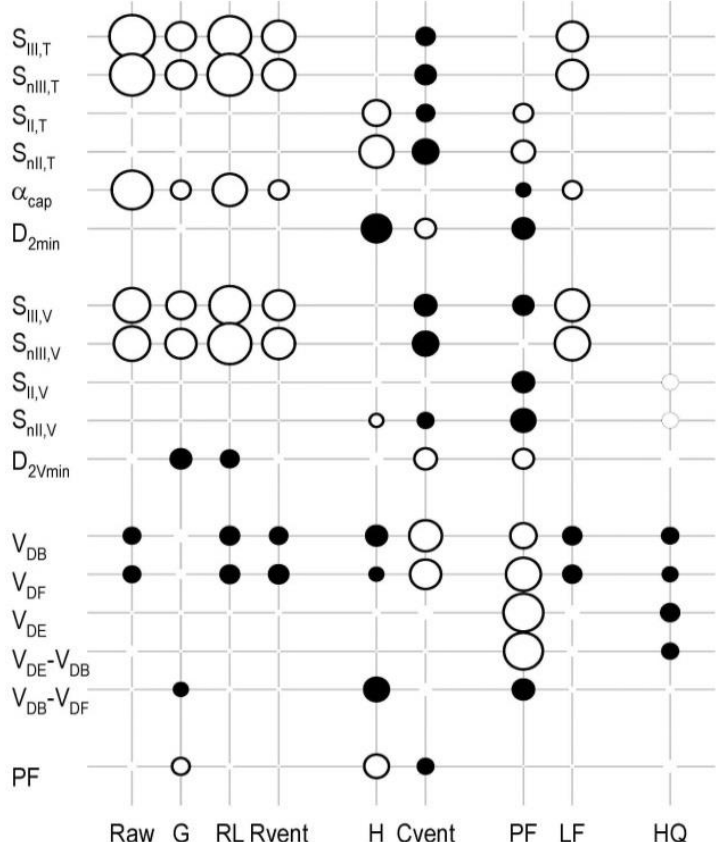

\section{After - Before CPB}

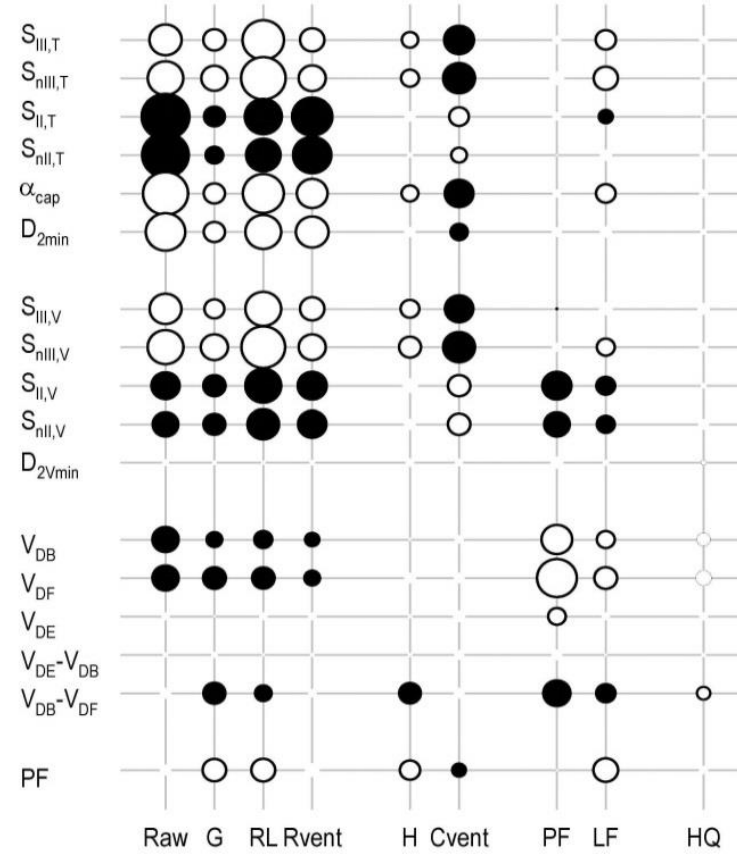

$\bigcirc$ positive correlation

negative correlation

Figure 5. Strengths of correlations between the lung mechanical parameters (x-axis) and those obtained by time and volumetric capnography, reflecting the absolute and normalized slopes, and the absolute values reflecting transitions, dead space and shunt fractions (y-axis). The sizes of the circles denote the magnitude of the Pearson correlation coefficient. Open circles: significant positive correlation; closed circles: significant negative correlation; no circles: no significant correlation. 
As concerns the relationships between the CPB-induced changes in the lung mechanical and capnographic indices (Fig. 5, bottom panel), the marked elevations in Raw correlated best $(\mathrm{p}=0.001)$ with the decreases in the phase-II slope parameters of the time capnogram ( $r=-0.72$ and -0.70 for $\mathrm{S}_{\mathrm{II}, \mathrm{T}}$ and $\mathrm{Sn}_{\mathrm{II}, \mathrm{T}}$, respectively; $\left.\mathrm{p}<0.0001\right)$. The CPB-induced airway narrowing was also reflected in the elevated phase-III slope parameters of the time and volumetric capnograms ( $r=0.49$ for both $S_{\text {III,T }}$ and $\left.S_{\text {III, }} ; \mathrm{p}<0.0001\right)$, and the curvature of the transition between the phases in the time domain $\left(r=0.6\right.$ for $\left.\mathrm{D}_{2 \min } ; \mathrm{p}<0.0001\right)$. The changes in the other mechanical parameters reflecting the tissue $(\mathrm{G})$ or total lung resistance ( $\mathrm{RL}$ or $\mathrm{R}_{\mathrm{vent}}$ ) displayed similar relationships with the alterations in the various capnographic indices after the $\mathrm{CPB}$. Assessment of the mild CPB-induced stiffening of the lung tissue also revealed statistically significant correlations between the changes in $\mathrm{C}_{\mathrm{vent}}$ and those in the phase-III slope parameters in both the time and volumetric capnograms ( $\mathrm{r}=-0.48$ for both $\mathrm{Sn}_{\text {III,T }}$ and $\left.\mathrm{Sn}_{\text {III, }} ; \mathrm{p}<0.0001\right)$. Neither the absolute values of HQ nor the changes following CPB exhibited close relationships with any other mechanical or capnographic indices; an association was observed with $\mathrm{V}_{\mathrm{DE}}$ before the $\mathrm{CPB}(\mathrm{r}=0.31 ; \mathrm{p}<0.0002)$.

The relationships between the initial fundamental lung mechanical and capnographic indices for the subgroups of patients based on starting HQ are depicted on Fig. 6A.

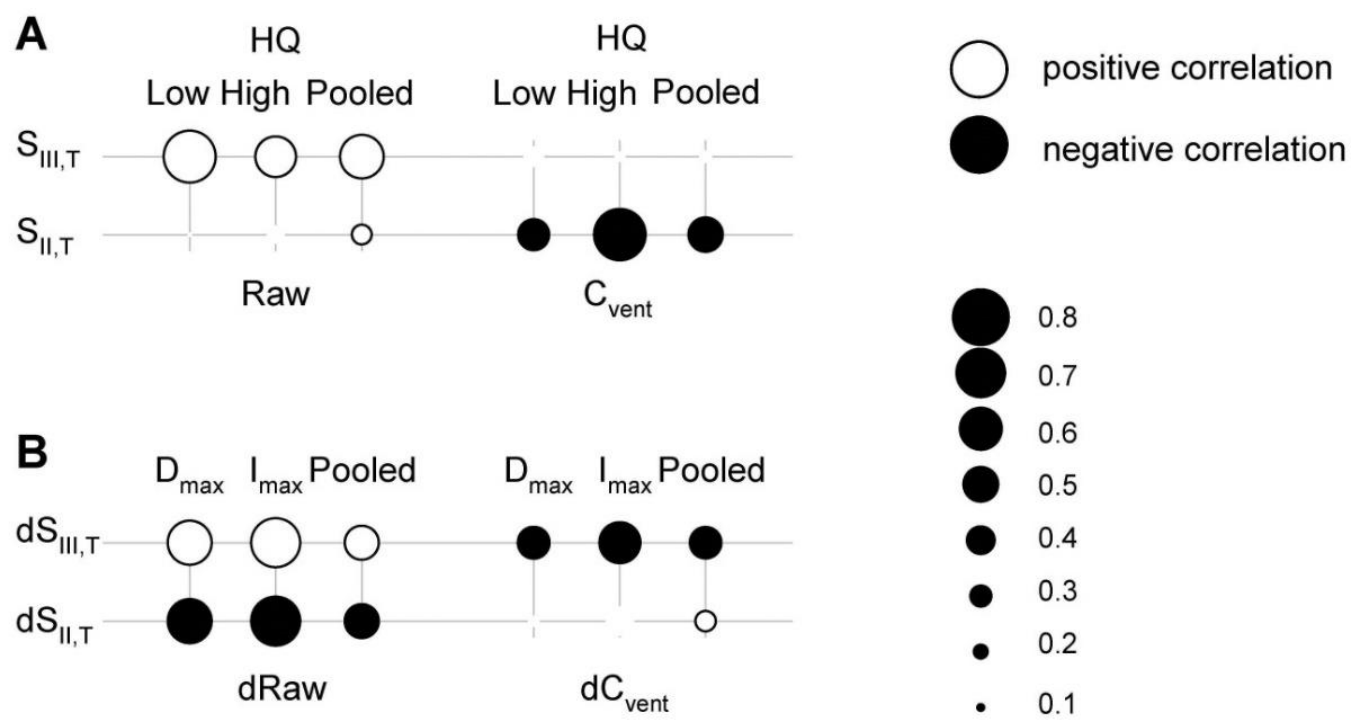

Figure 6. Strengths of correlations between the fundamental lung mechanical parameters (Raw and $\mathrm{C}_{\text {vent }}$; $\mathrm{x}$ axis) and phase II and III slopes obtained by time capnography (y-axis). Panel A: correlations between initial absolute values in the whole population (Pooled) and in subgroups with 25 percentile low and high initial HQ levels. Panel B: correlations between the changes in these parameters for the whole population (Pooled) and in subgroups with the highest 25 percentile increase $\left(I_{\max }\right)$, and lowest 25 percentile decrease $\left(D_{\max }\right)$ in HQ after surgery. The sizes of the circles denote the magnitude of the Pearson correlation coefficient. Open circles: 
significant positive correlation; closed circles: significant negative correlation; no circles: no significant correlation.

Strong positive significant correlations were observed between Raw and phase III slope parameters $(\mathrm{p}=0.002)$ and between $\mathrm{C}_{\mathrm{vent}}$ and phase II slope parameters independently of the subgroup allocation $(\mathrm{p}=0.001)$. The initial $\mathrm{C}_{\mathrm{vent}}-\mathrm{S}_{\mathrm{III}, \mathrm{T}}$ relationship was not significantly correlated $(\mathrm{p}=0.20)$, while the Raw-S $\mathrm{SII}_{\mathrm{T}}$ correlation appeared significant only for the pooled patient population $(\mathrm{p}=0.0045)$. The changes in Raw correlated to those in both slope variables $(\mathrm{p}<0.0001)$, whereas the alterations in $\mathrm{C}_{\text {vent }}$ were significantly related with those in phase III slopes ( $\mathrm{p}=0.023$, Fig. 6B).

The patients exhibited substantial inter-individual variability in the lung mechanical and capnographic parameters (Table 2), as reflected in the high coefficient of variation values before, after and the differences between after and before values.

\begin{tabular}{l|ccc} 
& Before & After & After-Before CPB \\
\hline Raw & 116 & 98 & 109 \\
$\mathrm{G}$ & 69 & 127 & 227 \\
$\mathrm{Sn}_{\text {II,T }}$ & 41 & 44 & 262 \\
$\mathrm{Sn}_{\text {III,T }}$ & 169 & 33 & 1163 \\
$\mathrm{~V}_{\mathrm{DF}}$ & 26 & 24 & 113 \\
$\mathrm{~V}_{\mathrm{DB}}$ & 25 & 24 & -134 \\
$\mathrm{~V}_{\mathrm{DE}}$ & 28 & 32 & -138 \\
$\mathrm{~V}_{\mathrm{DE}}-\mathrm{V}_{\mathrm{DB}}$ & 50 & 45 & 114 \\
& & &
\end{tabular}

Table 2. Coefficients of variations for the lung mechanical (Raw, $\mathrm{G}$ and $\mathrm{H}$ ) and capnograph-derived parameters $\left(\mathrm{Sn}_{\mathrm{II}, \mathrm{T}}, \mathrm{Sn}_{\mathrm{III}, \mathrm{T}}, \mathrm{V}_{\mathrm{DF}}, \mathrm{V}_{\mathrm{DB}}, \mathrm{V}_{\mathrm{DE}}, \mathrm{V}_{\mathrm{DE}}-\mathrm{V}_{\mathrm{DB}}\right)$.

The key parameters obtained by forced oscillations and capnography for the individual patients are demonstrated on Fig.7 (continuous thin lines), and for the group means (symbols with thick lines). 

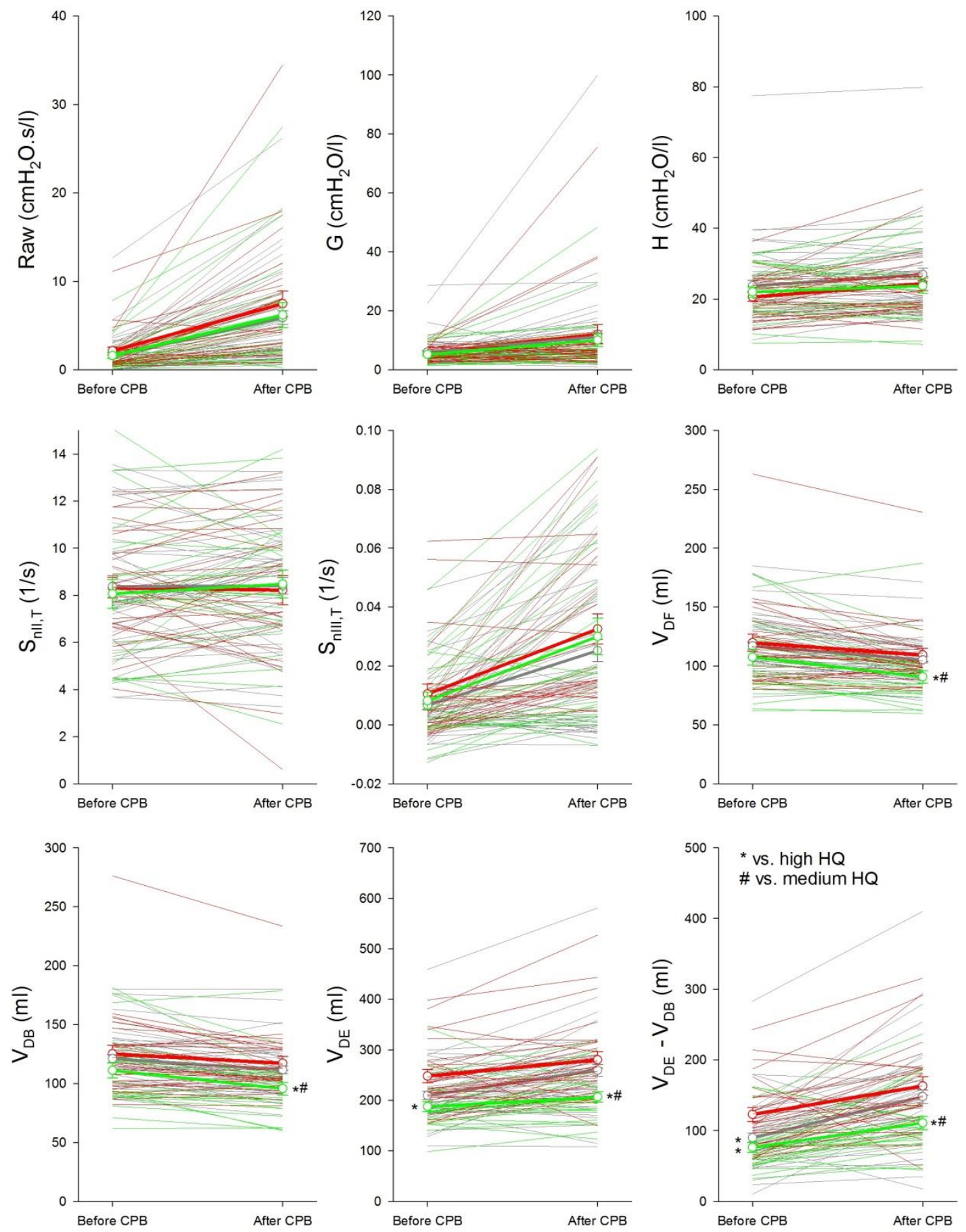

Figure 7. Lung mechanical (Raw, $G$ and $H$ ), and capnographic parameters $\left(S_{\mathrm{II}, \mathrm{T}}, \mathrm{Sn}_{\mathrm{III}, \mathrm{T}}, \mathrm{V}_{\mathrm{DF}}, \mathrm{V}_{\mathrm{DB}}, \mathrm{V}_{\mathrm{DE}}, \mathrm{V}_{\mathrm{DE}}-\right.$ $\mathrm{V}_{\mathrm{DB}}$ ) before and after $\mathrm{CPB}$, for the individual patients (continuous thin lines) and for the group means (thick lines). Red line: patients with the highest $25 \%$ starting HQ, grey line: patients with the medium $50 \%$ starting HQ, green line: patients with the lowest $25 \%$ starting HQ. 
Based on their starting pulmonary function, the cohort was divided into 3 groups: patients with the highest 25\% (red), the medium 50\% (grey) and the lowest 25\% HQ (green). There was no evidence for a statistical significance between the groups in the lung mechanical parameters (Raw, $\mathrm{G}$ and $\mathrm{H}$ ) and capnographic shape factors ( $\mathrm{Sn}_{\mathrm{II}, \mathrm{T}}$ and $\mathrm{Sn}_{\mathrm{III}, \mathrm{T}}$ ). This can be attributed to the complex pathophysiological processes involved in the gas exchange, including ventilation, perfusion and ventilation/perfusion. Accordingly, there is no direct link between individual lung mechanical or capnogram parameters with gas exchange indices. Conversely, capnographic parameters primarily affected by lung perfusion $\left(\mathrm{V}_{\mathrm{DF}}, \mathrm{V}_{\mathrm{DB}}, \mathrm{V}_{\mathrm{DE}}\right.$ and $\left.\mathrm{V}_{\mathrm{DE}}-\mathrm{V}_{\mathrm{DB}}\right)$ exhibit statistically significant differences in the different HQ groups.

The interdependence of the main shape factors obtained from the time capnogram with lung mechanical parameters representing the airway resistance (Raw) and lung elastance $(\mathrm{H})$ under the baseline conditions are demonstrated in Fig. 8.

The magnitude of $\mathrm{Sn}_{\text {III,T }}$ depends more on Raw than on $\mathrm{H}$ (Panel A), whereas the level of $\mathrm{Sn}_{\text {II,T }}$ appears to be determined primarily by $\mathrm{H}$, with lower correlations with Raw (Panel B). The capnographic parameters expressing the transition from phase II to phase III $\left(\mathrm{D}_{2 \min }\right)$ displayed stronger, but opposite dependence on $\mathrm{H}$ than on Raw (Panel C). 

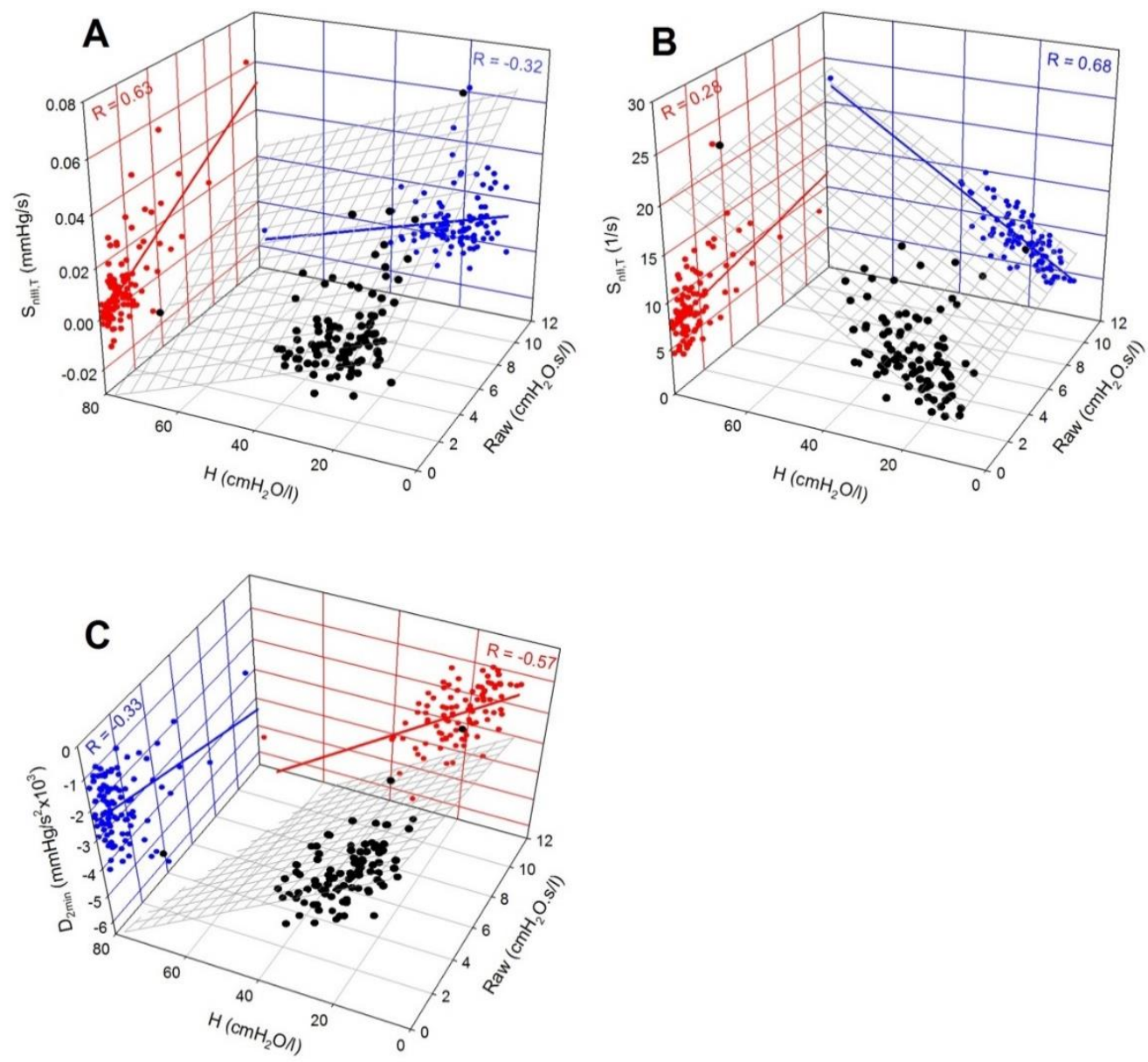

Figure 8. The correlation of the time capnographic shape factors $\left(\mathrm{Sn}_{\mathrm{II}, \mathrm{T}}, \mathrm{Sn}_{\mathrm{III}, \mathrm{T}}\right.$, and $\left.\mathrm{D}_{2 \mathrm{~min}}\right)$ and the lung mechanical parameters (Raw, $\mathrm{H}$ ) in three panels.

Study 2: Respiratory mechanics and the capnogram phases: importance of dynamic compliance

The changes in the respiratory mechanical parameters, the partial pressure of arterial oxygen $\left(\mathrm{PaO}_{2}\right)$ and the indices obtained from the capnograms with increasing PEEP in the 4 groups of patients are depicted in Fig. 9.

The statistical analyses revealed significant interactions between the group allocation and PEEP, demonstrating that the respiratory compliance exerted significant effects on the responses to PEEP in the forced oscillatory mechanical parameters ( $\mathrm{p}<0.001$ for Raw, $G$ and $\mathrm{H})$, for the Crs displayed by the respirator ( $\mathrm{p}<0.001), \mathrm{P}_{\mathrm{aO} 2}(\mathrm{p}=0.04)$, and the capnogram third phase slope variables $\left(\mathrm{p}<0.001\right.$ for $\mathrm{S}_{\text {III,T }}$ and $\mathrm{Sn}_{\text {III,T, }} \mathrm{p}=0.003$ for $\mathrm{S}_{\text {III,V }}$, and $\mathrm{p}=0.002$ and

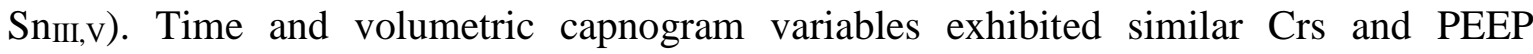
dependences, which is also reflected in the significant correlations between $S_{\text {III,T }}$ and $S_{\text {III,V }}$ 
in Groups HL ( $\mathrm{R}=0.4, \mathrm{p}=0.002), \mathrm{HC}(\mathrm{R}=0.79, \mathrm{p}<0.001), \mathrm{LC}(\mathrm{R}=0.45, \mathrm{p}=0.02)$ and $\mathrm{MC}$ $(\mathrm{R}=0.79, \mathrm{p}<0.001)$.
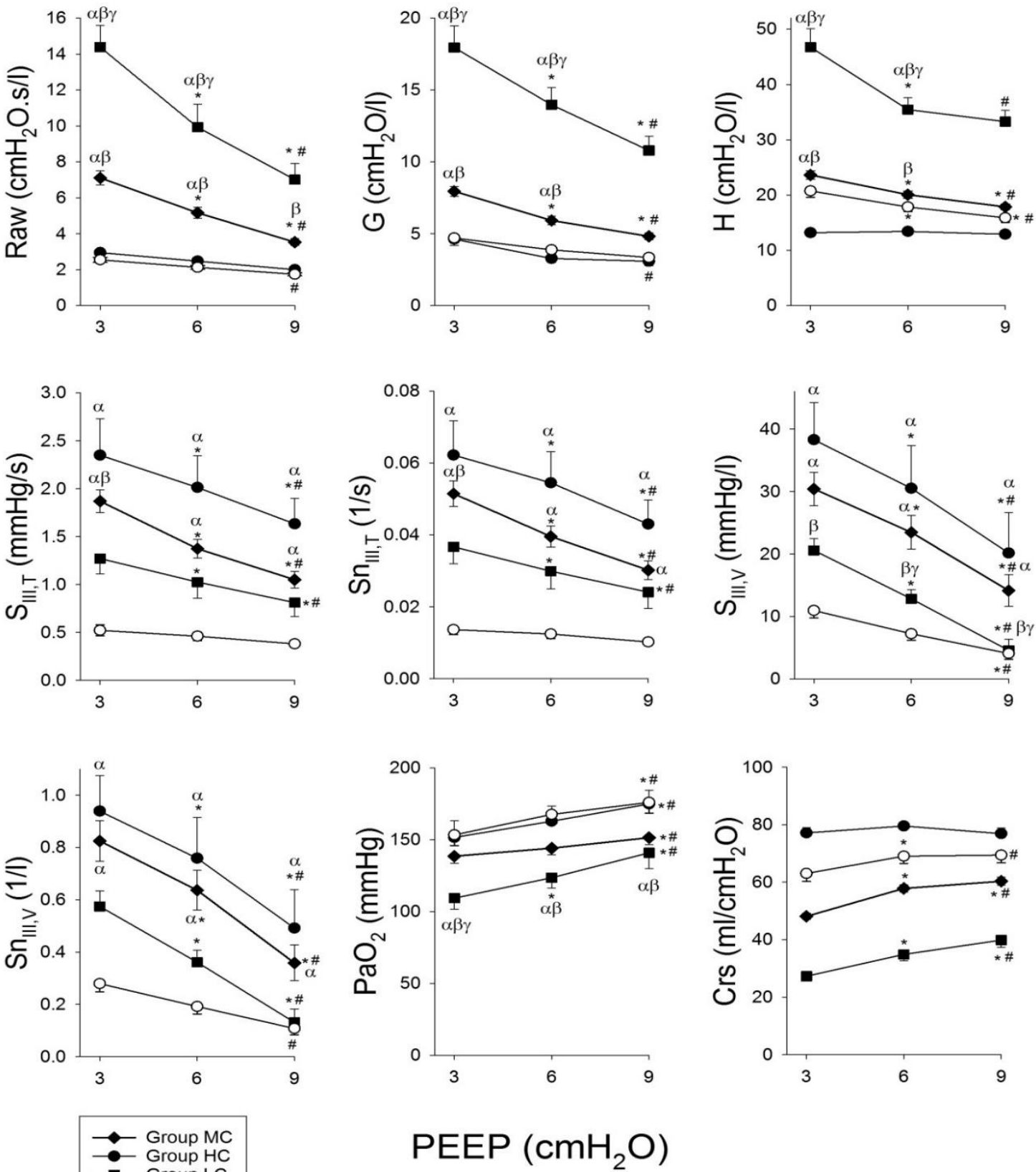

Figure 9. Forced oscillatory airway (Raw: airway resistance) and respiratory tissue (G: damping and H: elastance) mechanical parameters, the slope of the third phase of the capnogram as expressed in the time domain before $\left(\mathrm{S}_{\mathrm{III}, \mathrm{T}}\right)$ and after normalization for the mean expired $\mathrm{CO}_{2}\left(\mathrm{Sn}_{\mathrm{III}, \mathrm{T}}\right)$, or as a function of expired volume before $\left(\mathrm{S}_{\text {III,V }}\right)$ and after $\left(\mathrm{Sn}_{\text {III,V }}\right)$ normalization, partial pressure of oxygen in the arterial blood $\left(\mathrm{PaO}_{2}\right)$ and dynamic compliance (Crs) displayed by the respirator in patients with healthy lungs (Group HL), and in patients with respiratory symptoms with Crs in the lowest tenth percentile (Group LC), with Crs between the tenth and the ninetieth percentile (Group MC) and with Crs above the ninetieth percentile in (Group HC). *: $\mathrm{p}<0.05$ vs. the variable at the previous PEEP level; \#: $\mathrm{p}<0.05$ vs. a variable at a PEEP of $3 \mathrm{cmH}_{2} \mathrm{O} . \alpha: \mathrm{p}<0.05$ vs. Group HL within a PEEP; $\beta$ : $p<0.05$ vs. Group HC within a PEEP; $\gamma: p<0.05$ vs. Group MC within a PEEP. 
The greatest Raw, $\mathrm{G}, \mathrm{H}$ and the lowest $\mathrm{PaO}_{2}$ were observed for the patients in Group LC, and these patients generally exhibited the greatest response to PEEP. The patients in Group MC still exhibited elevated Raw, $\mathrm{G}$ and $\mathrm{H}$ with a more moderate, but still significant response to PEEP changes. The lowest forced oscillatory airway and tissue parameters and the greatest $\mathrm{P}_{\mathrm{aO} 2}$ were obtained in the patients in Groups $\mathrm{HL}$ and $\mathrm{HC}$, and their changes with PEEP were generally mild. The capnogram third phase indices were highest in Group HC and somewhat lower in Group MC, with both groups exhibiting marked decreases with increasing PEEP. The variables characterizing the third phase slopes from the capnogram were lowest in the patients in Group HL.

Fig. 10 depicts the relationship of Raw and $\mathrm{S}_{\mathrm{III}, \mathrm{T}}$ in the individual patients and the group means for the 4 protocol groups following the increases of PEEP.

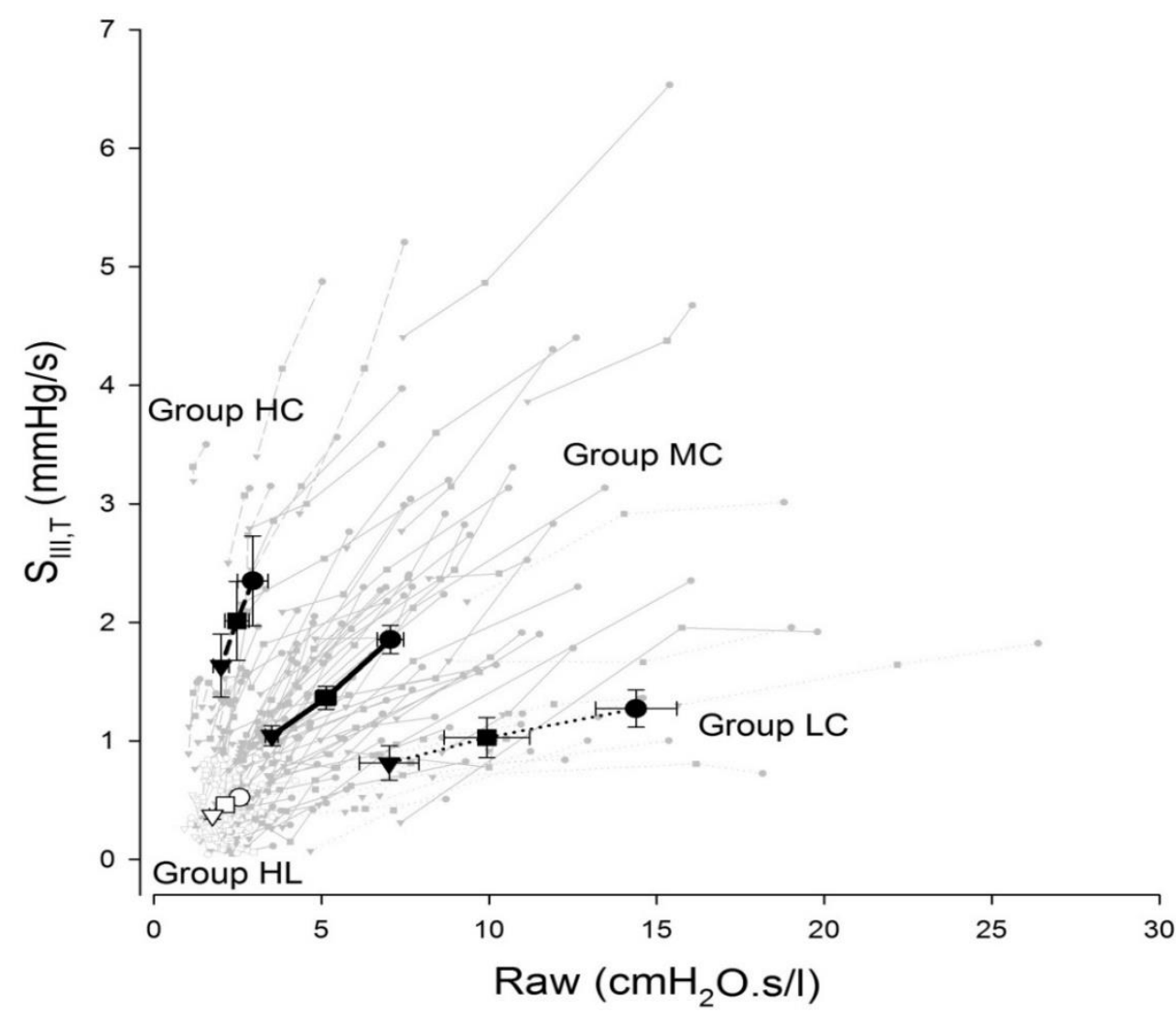

Figure 10. Relationship between forced oscillatory airway resistance (Raw) and phase III slope of time capnogram $\left(\mathrm{S}_{\mathrm{III}, \mathrm{T}}\right)$ at PEEP levels of $3(\mathbf{X}), 6(\boldsymbol{\nabla})$ and $9 \mathrm{cmH}_{2} \mathrm{O}(\boldsymbol{\Delta})$ in patients with healthy lungs (Group HL), and in patients with respiratory symptoms with Crs in the lowest tenth percentile in Crs (Group LC), with Crs between the tenth and ninetieth percentile (Group MC) and with Crs above the ninetieth percentile (Group $\mathrm{HC}$ ). Thin grey lines denote individual patients; thick black lines with symbols show group mean and SE values. 
In all patients, Raw and $\mathrm{S}_{\text {III,T }}$ underwent concomitant monotonous decreases with increasing PEEP, but marked differences were observed between the protocol groups in the relationships of these parameters. The marked decreases in the high initial Raw values were associated with substantially smaller drops in $\mathrm{S}_{\text {III }}$ in the patients in Group LC, whereas the PEEP-induced decreases in $\mathrm{S}_{\text {III }}$ were more pronounced than those in Raw in the patients in Group HC. The patients in Group MC exhibited an intermediate Raw- $\mathrm{S}_{\text {III,T }}$ relationship. This trend of association was observed in the patients in Group HL at markedly lower levels of Raw and $S_{\text {III. }}$

To examine the possible roles of obesity and lung congestion in the increased level of Crs, the effects of BMI and EF were considered (Fig. 11).

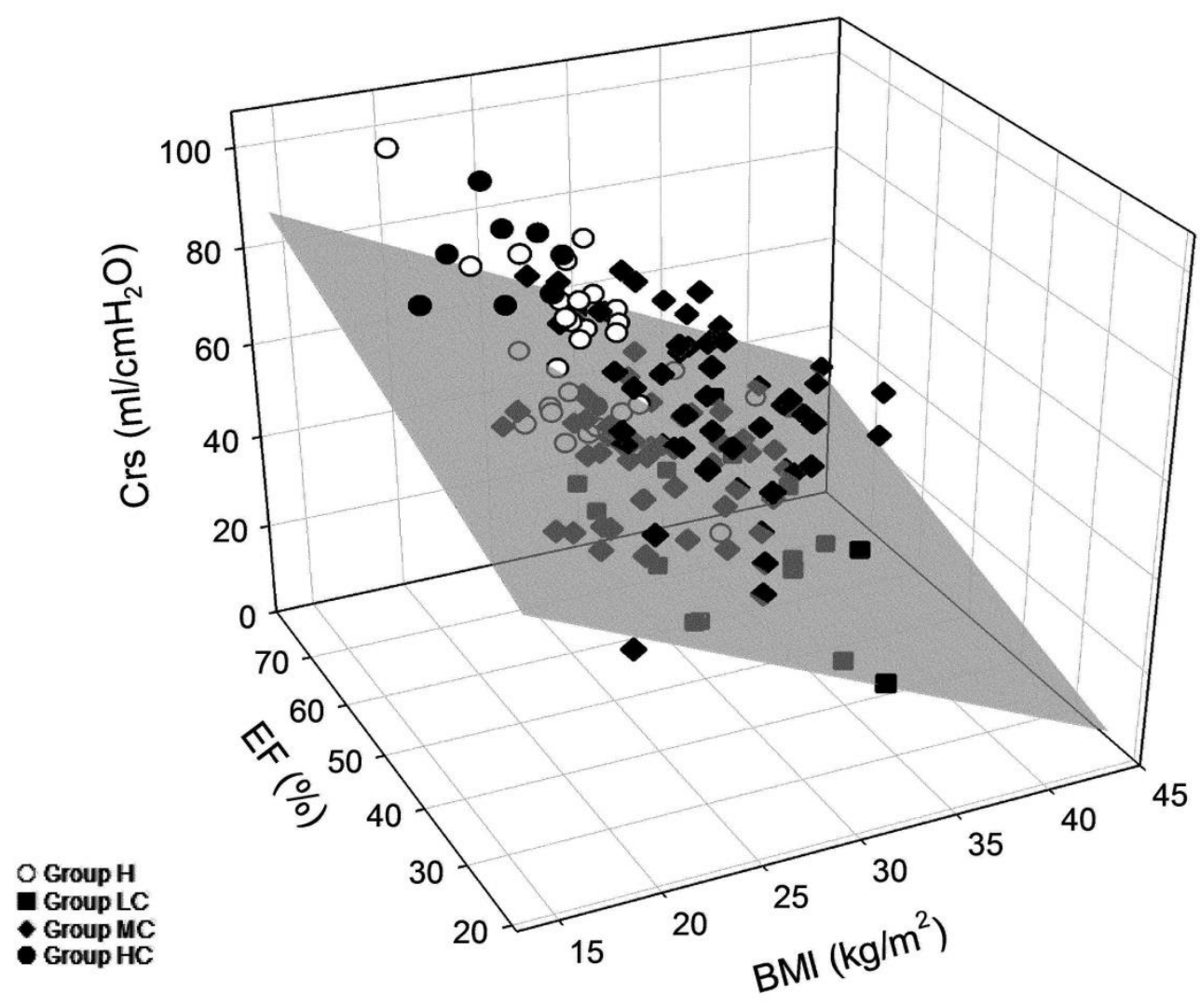

Figure 11. Effects of body mass index (BMI) and ejection fraction (EF) on dynamic respiratory compliance (Crs). The best fit plane is demonstrated with a mesh surface.

The patients in Group LC had significantly higher BMI $(\mathrm{p}<0.001)$ and/or lower EF ( $p<0.001)$ than those in Groups HL or HC, indicating that the low Crs was a consequence of restrictive changes resulting from obesity and/or heart failure leading to pulmonary 
congestion (multiple linear regression coefficient of $\mathrm{R}=0.58$ ). The important effects of BMI and $\mathrm{EF}$ on the group allocation was confirmed by the presence of a significant correlation $(\mathrm{R}=0.53, \mathrm{p}=0.005$ and $\mathrm{p}<0.0001$ for $\mathrm{EF}$ and $\mathrm{BMI}$, respectively $)$.

\section{DISCUSSION}

The data presented in this thesis revealed that the capnogram shape indices in ventilated patients are specifically influenced by the resistive and elastic parameters of the lungs or the respiratory system.

Under control conditions with open chest before CPB, the phase II slope of the capnogram is predominantly determined by the pulmonary elastic recoil. However, markedly elevated lung resistance after CPB can additionally worsen the capnogram phase II slope. Similarly, at baseline conditions before CPB, the phase III slope is shaped overwhelmingly by the airway resistance. However, strikingly elevated lung elastance after CPB can also decrease the capnogram phase III slope.

The dual effect of the resistive and elastic forces on phase III slope was further investigated under closed chest conditions before CPB with increasing PEEP as a challenge. Detailed analysis of the time capnogram revealed a strong association between Raw and $\mathrm{S}_{\text {III,T }}$ when the respiratory mechanics was altered by increasing PEEP. However, due to the substantial inter-individual variability in this relationship, $S_{\text {III,T }}$ provides useful information about alterations in the airway calibre only within an individual patient. Grouping of the patients based on their Crs revealed that i) the decrease in Raw with increasing PEEP is reflected in a small decrease in $\mathrm{S}_{\mathrm{III}, \mathrm{T}}$ in patients with low $\mathrm{Crs}$, ii) the increase in airway diameter with increasing PEEP was still reflected in a pronounced decrease in $S_{\text {III,T }}$ in patients with intermediate Crs, and iii) $\mathrm{S}_{\text {III,T }}$ was insensitive to changes in airway calibre when the Crs was high. In patients with high compliance, $S_{\text {III }}$ was the highest and the oxygenation was similar than in the healthy group. However, in patients with low Crs, the $\mathrm{S}_{\text {III }}$ was similar than that in the healthy patients, but their oxygenation was the worst. The relative homogeneous open alveoli of the lung parenchyma are emptying heterogeneously in $\mathrm{HC}$ group patients. In contrast, the relative heterogeneous open part of the alveolar compartment is emptying homogeneously in patients in the LC group. Therefore, low $\mathrm{S}_{\mathrm{III}}$ 
does not predict appropriate oxygenation alone in patients with low Crs, because it only reflects the alveolar emptying of the working lung.

Study 1: Capnographic parameters: correspondence with airway and tissue mechanics

\section{Phase III slope}

The results demonstrated significant increases in both $S_{\mathrm{III}, \mathrm{T}}$ and $\mathrm{S}_{\mathrm{III}, \mathrm{V}}$ immediately following the $\mathrm{CPB}$. The elevations also appeared after normalization to the $\mathrm{CO}_{2}$ concentration in the mixed expired gas (27) (Fig. 4). This finding differs from that observed previously in a smaller cohort of ventilated cardiac surgery patients, where no major changes were observed in the phase III slope after CPB (27). The discrepancy may be attributed to the more aggressive manoeuvers applied to recruit the lungs after the CPB in this earlier study, to the application of a higher PEEP ( 7 vs. $4 \mathrm{cmH}_{2} \mathrm{O}$ ) and to the somewhat delayed measurement time after the CPB (15 min vs. 5 min).

The phase III slope of the capnogram is considered to reflect the summation of the ventilation inhomogeneities relating to the working alveolar compartments with different time constants and the ventilation-perfusion mismatch as concerns the dead-space and/or intrapulmonary shunt. The overall and the regional lung emptying are determined by the opposite effects of Raw, $\mathrm{G}$ and the lung recoil tendencies. The role of the lung tissue stiffness decreases dynamically toward the end of expiration, and the elastic recoil affects $S_{\text {III }}$ in patients with low or high compliance. Raw, therefore, exerted the primary influence on the S III parameters before the CPB (Fig. 5 and 7), independent from the initial lung function (i.e. HQ; Fig. 6A). This finding is in accord with the postulate of the close link between the airway cross-sectional area and $\mathrm{S}_{\mathrm{III}}$, based on spirometric data obtained previously in spontaneously breathing patients $(50,53,57)$. Representing $(\mathrm{G})$ or incorporating lung parenchymal resistive component ( $\mathrm{RL}$ and $\mathrm{R}_{\mathrm{vent}}$ ) weakened the correlation substantially ( $<<0.0001 ;$ Fig. 5). This suggests that under baseline conditions the internal friction in the lung tissue does not exert a major effect on the capnographic $S_{\text {III }}$ indices, along with the lesser role of compliance. Following the $\mathrm{CPB}$, significant associations appeared between the overall resistive and capnographic phase III slope parameters, due to the greatly elevated tissue resistance (Fig. 5). Thus, an elevated $\mathrm{S}_{\text {III }}$ may indicate the presence of lung disorders affecting not only the airways, but also the tissue resistive properties, such as observed 
during interstitial edema in sepsis or cardiac failure (71). These phenomena are comprehensively confirmed by the significantly elevated concavity of the late expiratory flow (Fig. 3), the increase in $\mathrm{V}_{\mathrm{DE}}$ and the diminished HQ (Fig. 4).

\section{Phase II slope}

The phase II slope was decreased following the CPB in both the time and the volumetric capnograms. This agrees with the results of the only earlier study, where the changes in $S_{\text {II }}$ were measured 2 min after establishment of the full pulmonary blood flow (27). However, normalization of $\mathrm{S}_{\mathrm{II}}$ to a possible lower $\mathrm{CO}_{2}$ content of the expired gas (i.e. $\mathrm{Sn}_{\text {II }}$ ) after weaning from the CPB eliminated these changes (Fig. 4), because the intensity of axial gas mixing depends on the $\mathrm{CO}_{2}$ concentration (27).

Phase II of the capnogram represents the overall width of the moving airway-alveolar gas front and its slope may be explained by opposing effects. The heterogeneous start of lung emptying, the reduced airway lumen and increased tissue damping may all contribute to the decreases, whereas an elevated elastic recoil and a low alveolar $\mathrm{CO}_{2}$ content may counteract these changes in $S_{\text {II }}(27,37,50,53)$. Before the $C P B$, the correlation analyses of the $\mathrm{S}_{\text {II }}$ parameters indicated their close relationship with the elastic properties of the lungs (Fig. 5), independent from the starting gas exchange ability of the lungs (Fig. 6A). This finding is in accordance with a wider phase II observed previously in emphysematous patients $(36,72)$, and increases in $S_{\text {II }}$ after compliance elevation through recruitment manoeuvers (37). Since PF is determined more by the lung tissue stiffness ( $r=0.34 ; p<0.0001$ for $\mathrm{H}$ ) than by the airway caliber ( $\mathrm{r}=0.09 ; \mathrm{p}=0.34$ for Raw), the significant correlation of PF with the phase II capnographic indices may also be attributed to the influence of the lung elastance during early expiration.

Independent of the direction and magnitude of change in HQ, the CPB-induced changes in $\mathrm{S}_{\text {II }}$ exhibited close correlations with the markedly elevated Raw (Fig. 6B) and lung resistance parameters ( $\mathrm{RL}$ and $\mathrm{R}_{\mathrm{vent}} ;$ Fig. 5). This finding indicates that inhomogeneous airway constriction leads to a more sequential emptying of lung compartments with different $\mathrm{CO}_{2}$ content even at the beginning of expiration, and thereby widens the airway-alveolar gas front with subsequent decreases in the phase II slope. The loss of correlations between the 
changes in $\mathrm{S}_{\text {II,T }}$ and $\mathrm{C}_{\text {vent }}$ (Fig. 6B) may be due to the complex and opposing phenomena affecting $\mathrm{S}_{\mathrm{II}, \mathrm{T}}$, as described earlier.

\section{Capnographic parameters reflecting phase transitions}

The transition indices $\left(\alpha_{\text {cap }}, D_{2 m i n}\right.$ and $\left.D_{2 V \min }\right)$ reside in the same part of the capnogram, but their meanings are different. $\alpha_{\text {cap }}$ is the angle between $S_{\text {II }}$ and $S_{\text {III }}$, i.e. the relationship between the overall gas front and the alveolar gas volume, while $\mathrm{D}_{2 \min }$ and $\mathrm{D}_{2 \mathrm{Vmin}}$ are related to the internal surface of the moving $\mathrm{CO}_{2}$ diffusion front in the airways during expiration. The elevation observed in $\alpha_{\text {cap }}$ after the CPB reflects the combined alterations in $\mathrm{S}_{\text {II }}$ and $\mathrm{S}_{\text {III }}$, whereas both second derivative parameters approached zero after the CPB (Fig. 4), demonstrating blunted (less cornered) transitions between capnographic phases II and III. This finding may be attributed to the highly heterogeneous severe airway constriction that develops after the CPB, which blurs the resulting diffusion front, measured in the central airway. The close associations between $\mathrm{FEV}_{1}$ and the capnographic indices reflecting the phase II to phase III transition in spontaneously breathing patients is in accordance with this result (53). Our data further demonstrate that, in ventilated patients, the low compliance associated with the normal airway patency compresses the flow profile, resulting in a sharper phase II to phase III transition (Fig. 7 and 8). This finding reveals the sensitivity of $\mathrm{D}_{2 \min }$ and $\mathrm{D}_{2 \mathrm{~V} \text { min }}$ parameters to changes in lung compliance as opposed to $\alpha_{\mathrm{cap}}$, which demonstrates rather resistive properties (Fig. 5).

\section{Dead space and shunt parameters}

The anatomical dead space $\left(\mathrm{V}_{\mathrm{DF}}\right.$ ) was decreased slightly but consistently after the CPB (Fig. 4). Since this change was associated with marked increases in Raw and LF (Fig. 5, bottom), the compromised lumen of the conducting airways and/or their exclusion from the ventilation may explain this finding. The dead-space parameter incorporating the additional volume of the unperfused alveoli $\left(\mathrm{V}_{\mathrm{DB}}\right)$ followed very similar changing and correlation patterns, indicating the negligible unperfused, but ventilated alveolar compartment after the CPB. The bronchoconstriction resulting from the additive effects of SIRS and local hypocapnia may contribute to the low alveolar dead space. Conversely, supine position, surgery and CPB led to elevations in $\mathrm{V}_{\mathrm{DE}}$ (Fig. 4), suggesting a substantial enlargement of 
the volume of the not ventilated but perfused alveoli due to persistent atelectasis after the CPB $(73,74)$.

The difference between $V_{D E}$ and $V_{D B}$, which approximates the extent of the pulmonary shunt, was increased markedly after the CPB (Fig. 4). It is noteworthy that $V_{D E}-V_{D B}$ exhibited parallel changes and a significant correlation $(r=0.47 ; p<0.0001)$ with the shunt fraction obtained from the classical shunt equation (Qs/Qt), highlighting the additional usefulness of volumetric capnography in the assessment of the intrapulmonary shunt (Fig. 4).

\section{Methodological aspects}

While patients with severe cardiopulmonary disorders were excluded from the present study, the pulmonary status of the participating subjects varied widely from relatively healthy lungs to obstructive and restrictive disorders. Such inter-individual variety of pulmonary symptoms with additional demographic and anthropometric differences is expected to occur in all health-care units providing ventilatory support. Therefore, this feature of the study is particularly favourable and also facilitates the performance of the correlation analyses.

It is also noteworthy that the results represent an open chest condition. Significant alteration in lung-thorax dynamics is expected to influence both the capnography indices and forced oscillatory data reflecting airway and tissue mechanics. The capnogram parameters are determined by the heterogeneity of the lungs, geometry of the airway tree and the forces exerted by the tissue resistive and elastic properties of the lungs and the chest wall. While our study allows an insight into the mechanisms coupling the capnogram and mechanical parameters, a further study in intact chest patients may be needed to generalize our findings.

A further important methodological aspect of the present study is related to the use of correlation analyses to assess the associations between parameters obtained by two different techniques. As a general rule, the existence of significant correlations between variables is necessary, but not sufficient to imply a causal relationship. In the present study, the lung mechanical and capnographic parameters are linked to each other through local common mechanisms governing lung emptying. Furthermore, the individual correlation results from consistent physiological and clinical findings. These considerations verify that causation can be inferred with great certainty. 
Study 2: Respiratory mechanics and the capnogram phases: importance of dynamic compliance

The extent of emptying of lung compartments containing various $\mathrm{CO}_{2}$ concentrations during mechanical ventilation and the shape of the resulting capnogram are determined by the airway geometry (i.e. the resistance) and the elastic recoil of the respiratory tissues (i.e. the driving pressure). Whereas the former has been investigated extensively $(33,34,52,57,58)$, the importance of the latter factor remained unknown. While the time capnogram is most commonly used in clinical practice during mechanical ventilation, distinction of the second and third phases is not always trivial from the time domain analyses and this approach also excludes the consideration of the absolute concentration of $\mathrm{CO}_{2}$ in the expired gas. Therefore, we also performed volumetric capnography in a subgroup of patients and normalized the capnogram third phase slope. The similar picture of these different slopes and the significant correlation between them demonstrates that the $S_{\text {III,T }}$ used in clinical practice can provide relevant information about lung emptying. With the aim of acquiring a general picture, the bedside Crs was used in the present study to group the patients. A strong correlation was earlier demonstrated between the respiratory elastance derived from $\mathrm{H}$ and the Crs (75), which justifies the choice of Crs as an appropriate indicator of the respiratory recoil.

We formed four groups with regard to the clinical symptoms (healthy lungs) and the Crs values (diseased lungs with low, medium or high Crs). As expected, the variables reflecting resistive behavior were lowest in the Group HL and they had intermediate $\mathrm{H}$ and Crs, permitting fast emptying of the relatively homogeneous lungs, which then results in low capnogram third phase slope, and good $\mathrm{PaO}_{2}$ (Fig. 9). The increase of PEEP to $6 \mathrm{cmH}_{2} \mathrm{O}$ caused no further improvement. The slight, but significant decreases in Raw and $\mathrm{H}$ and increases in $\mathrm{Crs}$ and $\mathrm{P}_{\mathrm{aO} 2}$ at PEEP $9 \mathrm{cmH}_{2} \mathrm{O}$ may be a consequence of lung recruitment. The lack of decrease in the third phase slope indicates that this opening was relatively uniform in the lung periphery.

The patients in Group HC exhibited similar resistive properties to those in Group HL. However, the high Crs and low $\mathrm{H}$ may be a consequence of the loss of elastic recoil in the respiratory tissues, most probably due to emphysematous destruction, which was present in the vast majority of the patients in this group (Table 1). The presence of ventilation 
heterogeneities is also apparent from the highest third phase slope indices. These can be explained by the existence of peripheral lung units with different small airway calibers and local time constants, resulting in a heterogeneous working lung. This structure leads to gas compartments containing variable $\mathrm{CO}_{2}$ concentrations and also results in different local expiratory flows (43). These phenomena contribute to the sequential emptying of the lung periphery in time, which then increases the time domain and volumetric $S_{\text {III }}$ values $(22,28,29,33,76)$. Since Raw reflects mainly the flow resistance of the central conducting airways $(60,75,77)$, this parameter is not able to detect such alterations in the presence of emphysematous changes (78). This pathology diminishes predominantly the expiratory flow, while the filling of the lung during mechanical ventilation may remain unaffected or even increased, explaining why $\mathrm{P}_{\mathrm{aO} 2}$ was close to normal. The elevation of PEEP in Group HC decreased $\mathrm{G}$, which is a prerequisite of decreased ventilation heterogeneities with alveolar recruitment $(79,80)$, reflecting in lower capnogram third phase slopes, $\mathrm{H}$, higher Crs and better $\mathrm{PaO}_{2}$ (34).

The worst respiratory mechanics and the lowest $\mathrm{P}_{\mathrm{aO}}$ were observed in the patients in Group LC. Despite this striking difference, $S_{\text {III }}$ expressed in time or by volumetry did not differ significantly from those observed in Group HL. This leads to the important observation that even hypoxemia may be associated with a medium level of capnogram third phase slopes, which corresponds to the limited value of capnography in the assessment of adequate blood oxygenation in this pathology $(81,82)$. These results can most probably be attributed to the presence of lung regions that remain closed throughout the entire ventilator cycle, leading to some relatively open and fairly uniform working lung units and other, permanently closed, atelectatic lung units. In other words, the closing capacity in these lungs is expected to be higher than the sum of the functional residual capacity and the tidal volume. The persistent lung volume loss with subsequent decrease in the overall airway crosssectional area is probably reflected in the substantially elevated Raw. Since PEEP elevation may be able to reopen these atelectases, the involvement of these phenomena is substantiated by the most pronounced decreases in the mechanical parameters with increasing PEEP resulting in lower $\mathrm{S}_{\mathrm{III}}$ and elevated $\mathrm{PaO} 2$, which corresponds to earlier results on similar stiff lungs (22). Our results confirm previous clinical observations $(32,34,77)$ that this pathophysiology can be triggered by obesity and/or lung congestion arising from a poor EF (Table 1). Taking into account the individual and the combined effects of BMI and EF 
revealed that low EF or high BMI themselves may be responsible for the compromised Crs. However, the combination of such pathologies exerts additional detrimental effects that lower Crs even more dramatically (Fig. 11).

Group MC comprised patients with pulmonary pathologies with an intermediate Crs, a cohort that can be characterized by somewhat elevated airway and respiratory tissue parameters, and ventilation heterogeneities reflected in abnormally high capnography slope characteristics at a PEEP of $3 \mathrm{cmH}_{2} \mathrm{O}$. This variable and the intermediate response to PEEP can be explained by concomitant presence of phenomena existing in Groups HC and LC, i.e. combined effects of expiratory flow limitation and persistent atelectases.

The overall Raw $-S_{\text {III,T }}$ relationship was not strong enough to predict the value of Raw from $\mathrm{S}_{\mathrm{III}, \mathrm{T}}$ (Fig. 10), in agreement with previous findings $(58,59)$. However, the changes in $S_{\text {III,T }}$ within an individual patient were appropriate for an assessment or revealing trends of the altered Raw. It should be noted that the Raw $-\mathrm{S}_{\mathrm{III}, \mathrm{T}}$ relationship within a patient was highly dependent on the elastic recoil of the respiratory system. In the case of a small Crs, a minor change in $\mathrm{S}_{\text {III,T }}$ may reflect major alterations in airway patency. In contrast, large alterations in $\mathrm{S}_{\mathrm{III}, \mathrm{T}}$ may still be associated with small variations in Raw if Crs is high. This finding may explain the controversy in the literature concerning the presence or absence of a correlation between lung function parameters and capnogram indices $(43,52,53,57)$.

The limitations of this study relate to the possible presence of complex cardiopulmonary pathologies within a given patient. The coexistence of opposing factors such as emphysematous changes and a poor left ventricular function precludes identification of the individual effects of pulmonary diseases on the course of the capnogram. An additional aspect is that the surgery did not allow a more time-consuming randomization of the PEEP levels. However, care was taken to provide sufficient time following a change in conditions so that equilibrium was reached, similarly to that allowed following PEEP changes in severe COPD patients (79). Another methodological limitation is related to the complex effects of PEEP including modification of the lung perfusion (27), increased FRC $(63,83)$, which may all bias the changes in $S_{\text {III }}$ and/or the mechanical parameters. However, our results are consistent even on PEEP $3 \mathrm{cmH}_{2} \mathrm{O}$ alone and the PEEP changes can be considered as reinforcement of the results and mechanisms that existed already at the lower PEEP. Auto-PEEP may be another important factor imposing a potential error with this bias 
being the most apparent in the patients with high Raw (i.e. Group LC) or low driving pressure and compromised emptying of emphysematous destructed alveoli (i.e. Group HC). Excluding the auto-PEEP would even enhance the Raw dependence with PEEP, since Raw would theoretically be even higher if auto-PEEP would have been ruled out. Another important feature of the present study is the use of Crs to separate the study groups. Since this parameter incorporates lung and chest wall properties, a separate assessment of which of these compartments are responsible for the altered elastic recoil of the respiratory system is not possible.

\section{SUMMARY AND CONCLUSION}

The present thesis describes studies focusing on capnography providing overlapping technical and vital physiological information about ventilation, circulation and metabolic processes. This technique is an often used, but not completely utilized monitoring method in the clinical practice. The data presented in this thesis revealed that the capnogram shape indices in ventilated patients are specifically influenced by the resistive and elastic parameters of the lungs or the respiratory system.

Study 1: Capnographic parameters: correspondence with airway and tissue mechanics

We characterized the relationships between the time or volumetric capnographic parameters and the lung mechanical indices reflecting the airway and the lung tissue viscoelastic properties in cardiac surgery patient underwent open heart surgery. Since the elastic forces are maximal at high lung volumes at early exhalation, the lung tissue stiffness predominantly determines the capnographic parameters in the early phase of expiration. Thus, in the vast majority of the cases, the phase-II slope of the capnogram is predominantly determined by pulmonary elastic recoil. Conversely, the resistive properties of the lungs become increasingly important during the later phase of expiration at lower lung volumes, and thus, the phase III slope is shaped overwhelmingly by the airway resistance. However, markedly elevated lung resistance additionally worsens the capnogram phase II slope. Similarly, severely compromised lung elastance also distorts the capnogram phase III slope. 
Study 2: Respiratory mechanics and the capnogram phases: importance of dynamic compliance

Measurement of the respiratory mechanics and analysis of the capnogram slope demonstrated that changes in $\mathrm{S}_{\mathrm{III}}$ expressed in time or volume domain provide useful information concerning alterations in airway calibre, but only within an individual patient. The assessment of $S_{\text {III,T }}$ during mechanical ventilation may be of value for bedside monitoring of the changes in airway resistance, but its sensitivity depends on the elastic recoil of the respiratory system. SIII,T exhibits high sensitivity to detect changes in the airway resistance in case of high Crs, when the lung emptying is governed primarily by the small airway and alveolar geometry. In cases of stiff respiratory tissues, however, $\mathrm{S}_{\mathrm{III}, \mathrm{T}}$ displays low sensitivity in indicating changes in airway caliber, when the lung emptying is determined by the high elastic recoil and depends less on the small airway geometry. The relatively low $\mathrm{S}_{\text {III,T }}$ may result from homogenous alveolar emptying of an overinflated, but decreased fraction of lung parenchyma and may coincide with the compromised $\mathrm{P}_{\mathrm{aO} 2}$ in these

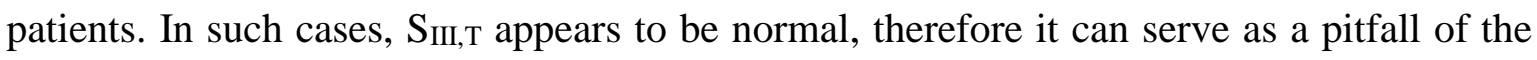
ventilation monitoring. A low and apparently physiological $\mathrm{S}_{\text {III,T }}$ does not predict appropriate oxygenation alone. Thus, the shape of the capnogram should always be evaluated bedside in conjunction with Crs. The joint assessment of the capnogram and the respiratory mechanics is of particular importance in clinical situations when patients with a high BMI and/or a compromised left ventricular function are anesthetized and ventilated.

Since computational methods could be incorporated into the modern anaesthesia machines to quantify capnographic shape factors, these parameters together with the traditional bedside mechanical indices has the promise to improve differential diagnoses and advance guiding respiratory therapy. Overall, our results suggest that all the important clinical capacity of the capnography can be exploited during ventilation the patients in the anaesthesia and intensive therapy. 


\section{ACKNOWLEDGEMENTS}

I thank Dr Barna Babik for introducing me to this research area and for his continuous guidance and support. His excellent advices have been essential in the development of my research.

I am also very grateful to Dr Ferenc Peták for his highly valuable help in conducting my work.

I wish to express my gratitude to Dr József Tolnai, Dr Ádám Balogh, Dr Gergely Fodor for helping me find my way in analysing the capnogram and oscillatory data, and Lajos Vígh to their excellent technical assistance.

I thank Professor Zsolt Molnár, the Head of Department of Anaesthesiology and Intensive Therapy in University of Szeged for supporting my research.

I would like to thank Dr. Gábor Bogáts, the Head of Department of Cardiac Surgery in University of Szeged and all his staff for their contribution and help throughout the construction of the studies.

This work was funded by a Hungarian Basic Scientific Research Grant (OTKA K81179). This research was supported by the European Union and the State of Hungary, co-financed by the European Social Fund in the framework of TÁMOP 4.2.4. A/2-11-1-2012-0001 'National Excellence Program'. 


\section{References}

1. Bouguer P. Essai d'optique sur la gradation de la lumière Paris, France, 1729.

2. Lambert JH. Photometria sive de mensura et gradibus luminis, colorum et umbrae [Photometry, or, On the measure and gradations of light, colors, and shade] Augsburg, Germany, 1760.

3. Beer. Bestimmung der Absorption des rothen Lichts in farbigen Flüssigkeiten (Determination of the absorption of red light in colored liquids), 1852.

4. Ortega R, Connor C, Kim S, Djang R, Patel K. Monitoring ventilation with capnography. N Engl J Med 2012;367:e27.

5. Pascucci RC, Schena JA, Thompson JE. Comparison of a sidestream and mainstream capnometer in infants. Crit Care Med 1989;17:560-2.

6. MB J. Mainstream or sidestream capnography, Respironics, Inc., www.oem.respironics.com 2002.

7. Szaflarski NL, Cohen NH. Use of capnography in critically ill adults. Heart Lung 1991;20:363-72.

8. Ward KR, Yealy DM. End-tidal carbon dioxide monitoring in emergency medicine, Part 2: Clinical applications. Acad Emerg Med 1998;5:637-46.

9. Lawson D, Jelenich S. Capnographs: a new operating room pollution hazard? Anesth Analg 1985;64:378.

10. Breen PH, Isserles SA, Harrison BA, Roizen MF. Simple computer measurement of pulmonary VCO2 per breath. J Appl Physiol (1985) 1992;72:2029-35.

11. Breen PH, Mazumdar B, Skinner SC. Capnometer transport delay: measurement and clinical implications. Anesth Analg 1994;78:584-6.

12. From RP, Scamman FL. Ventilatory frequency influences accuracy of end-tidal CO2 measurements. Analysis of seven capnometers. Anesth Analg 1988;67:884-6.

13. Anderson CT, Breen PH. Carbon dioxide kinetics and capnography during critical care. Crit Care 2000;4:207-15.

14. Bhavani-Shankar K, Philip JH. Defining segments and phases of a time capnogram. Anesth Analg 2000;91:973-7.

15. Thompson JE, Jaffe MB. Capnographic waveforms in the mechanically ventilated patient. Respir Care 2005;50:100-8; discussion 8-9. 
16. Walsh BK, Crotwell DN, Restrepo RD. Capnography/Capnometry during mechanical ventilation: 2011. Respir Care 2011;56:503-9.

17. Bhavani-Shankar

$\mathrm{K}$. http://www.capnography.com/new/indexphp?option=com_content\&view=article\&i $\underline{\mathrm{d}=73 \& \text { ltemid }=96 .}$.

18. Romero PV, Rodriguez B, de Oliveira D, Blanch L, Manresa F. Volumetric capnography and chronic obstructive pulmonary disease staging. Int $\mathrm{J}$ Chron Obstruct Pulmon Dis 2007;2:381-91.

19. Blanch L, Romero PV, Lucangelo U. Volumetric capnography in the mechanically ventilated patient. Minerva Anestesiol 2006;72:577-85.

20. Tusman G, Scandurra A, Bohm SH, Suarez-Sipmann F, Clara F. Model fitting of volumetric capnograms improves calculations of airway dead space and slope of phase III. J Clin Monit Comput 2009;23:197-206.

21. Romero PV, Lucangelo U, Lopez Aguilar J, Fernandez R, Blanch L. Physiologically based indices of volumetric capnography in patients receiving mechanical ventilation. Eur Respir J 1997;10:1309-15.

22. Tusman G, Suarez-Sipmann F, Bohm SH, Borges JB, Hedenstierna G. Capnography reflects ventilation/perfusion distribution in a model of acute lung injury. Acta Anaesthesiol Scand 2011;55:597-606.

23. Merilainen P, Hanninen H, Tuomaala L. A novel sensor for routine continuous spirometry of intubated patients. J Clin Monit 1993;9:374-80.

24. Fletcher R, Jonson B. Deadspace and the single breath test for carbon dioxide during anaesthesia and artificial ventilation. Effects of tidal volume and frequency of respiration. Br J Anaesth 1984;56:109-19.

25. Bhavani-Shankar K, Kumar AY, Moseley HS, Ahyee-Hallsworth R. Terminology and the current limitations of time capnography: a brief review. J Clin Monit $1995 ; 11: 175-82$.

26. Bhavani-Shankar K, Moseley H, Kumar AY, Delph Y. Capnometry and anaesthesia. Can J Anaesth 1992;39:617-32.

27. Tusman G, Areta M, Climente C, Plit R, Suarez-Sipmann F, Rodriguez-Nieto MJ, Peces-Barba G, Turchetto E, Bohm SH. Effect of pulmonary perfusion on the slopes of single-breath test of CO2. J Appl Physiol (1985) 2005;99:650-5. 
28. Crawford AB, Makowska M, Paiva M, Engel LA. Convection- and diffusiondependent ventilation maldistribution in normal subjects. J Appl Physiol (1985) 1985;59:838-46.

29. Dutrieue B, Vanholsbeeck F, Verbanck S, Paiva M. A human acinar structure for simulation of realistic alveolar plateau slopes. J Appl Physiol (1985) 2000;89:185967.

30. Verbanck S, Paiva M. Model simulations of gas mixing and ventilation distribution in the human lung. J Appl Physiol (1985) 1990;69:2269-79.

31. Fletcher R, Jonson B, Cumming G, Brew J. The concept of deadspace with special reference to the single breath test for carbon dioxide. Br J Anaesth 1981;53:77-88.

32. Hoffbrand BI. The expiratory capnogram: a measure of ventilation-perfusion inequalities. Thorax 1966;21:518-23.

33. Stromberg NO, Gustafsson PM. Ventilation inhomogeneity assessed by nitrogen washout and ventilation-perfusion mismatch by capnography in stable and induced airway obstruction. Pediatr Pulmonol 2000;29:94-102.

34. Bohm SH, Maisch S, von Sandersleben A, Thamm O, Passoni I, Martinez Arca J, Tusman G. The effects of lung recruitment on the Phase III slope of volumetric capnography in morbidly obese patients. Anesth Analg 2009;109:151-9.

35. Frankenfield DC, Alam S, Bekteshi E, Vender RL. Predicting dead space ventilation in critically ill patients using clinically available data. Crit Care Med 2010;38:28891.

36. Kars AH, Bogaard JM, Stijnen T, de Vries J, Verbraak AF, Hilvering C. Dead space and slope indices from the expiratory carbon dioxide tension-volume curve. Eur Respir J 1997;10:1829-36.

37. Tusman G, Bohm SH, Suarez-Sipmann F, Turchetto E. Alveolar recruitment improves ventilatory efficiency of the lungs during anesthesia. Can J Anaesth 2004;51:723-7.

38. Veronez L, Moreira MM, Soares ST, Pereira MC, Ribeiro MA, Ribeiro JD, Terzi RG, Martins LC, Paschoal IA. Volumetric capnography for the evaluation of pulmonary disease in adult patients with cystic fibrosis and noncystic fibrosis bronchiectasis. Lung 2010;188:263-8.

39. Poppius H. Expiratory, CO2 curve in pulmonary diseases. Scand J Respir Dis 1969;50:135-46. 
40. Bohr C. Über die Lungenatmung. Skan Arch Physiol 1891;53:236-8.

41. Hedenstierna G, Sandhagen B. Assessing dead space. A meaningful variable? Minerva Anestesiol 2006;72:521-8.

42. Enghoff H. Volumen inefficax. Uppsala Laekareforen Forh 1938;44:191-218.

43. Krauss B, Deykin A, Lam A, Ryoo JJ, Hampton DR, Schmitt PW, Falk JL. Capnogram shape in obstructive lung disease. Anesth Analg 2005;100:884-8, table of contents.

44. Neumar RW, Otto CW, Link MS, Kronick SL, Shuster M, Callaway CW, Kudenchuk PJ, Ornato JP, McNally B, Silvers SM, Passman RS, White RD, Hess EP, Tang W, Davis D, Sinz E, Morrison LJ. Part 8: adult advanced cardiovascular life support: 2010 American Heart Association Guidelines for Cardiopulmonary Resuscitation and Emergency Cardiovascular Care. Circulation 2010;122:S729-67.

45. Chopin C, Fesard P, Mangalaboyi J, Lestavel P, Chambrin MC, Fourrier F, Rime A. Use of capnography in diagnosis of pulmonary embolism during acute respiratory failure of chronic obstructive pulmonary disease. Crit Care Med 1990;18:353-7.

46. Verschuren F, Liistro G, Coffeng R, Thys F, Roeseler J, Zech F, Reynaert M. Volumetric capnography as a screening test for pulmonary embolism in the emergency department. Chest 2004;125:841-50.

47. Cheifetz IM, Myers TR. Respiratory therapies in the critical care setting. Should every mechanically ventilated patient be monitored with capnography from intubation to extubation? Respir Care 2007;52:423-38; discussion 38-42.

48. Hall D, Goldstein A, Tynan E, Braunstein L. Profound hypercarbia late in the course of laparoscopic cholecystectomy: detection by continuous capnometry. Anesthesiology 1993;79:173-4.

49. Poirier MP, Gonzalez Del-Rey JA, McAneney CM, DiGiulio GA. Utility of monitoring capnography, pulse oximetry, and vital signs in the detection of airway mishaps: a hyperoxemic animal model. Am J Emerg Med 1998;16:350-2.

50. Krauss B. Capnography as a rapid assessment and triage tool for chemical terrorism. Pediatr Emerg Care 2005;21:493-7.

51. Yang Y, Huang Y, Tang R, Chen Q, Hui X, Li Y, Yu Q, Zhao H, Qiu H. Optimization of positive end-expiratory pressure by volumetric capnography variables in lavageinduced acute lung injury. Respiration 2014;87:75-83. 
52. Yaron M, Padyk P, Hutsinpiller M, Cairns CB. Utility of the expiratory capnogram in the assessment of bronchospasm. Ann Emerg Med 1996;28:403-7.

53. You B, Peslin R, Duvivier C, Vu VD, Grilliat JP. Expiratory capnography in asthma: evaluation of various shape indices. Eur Respir J 1994;7:318-23.

54. Merry AF, Cooper JB, Soyannwo O, Wilson IH, Eichhorn JH. International Standards for a Safe Practice of Anesthesia 2010. Can J Anaesth 2010;57:1027-34.

55. Guidelines for Patient Care in Anesthesiology. http://www.asahq.org/resources/standards-and-guidelines: American Society of Anesthsiologists, 2011.

56. Veronez L, Pereira MC, da Silva SM, Barcaui LA, De Capitani EM, Moreira MM, Paschoal IA. Volumetric capnography for the evaluation of chronic airways diseases. Int J Chron Obstruct Pulmon Dis 2014;9:983-9.

57. Nik Hisamuddin NA, Rashidi A, Chew KS, Kamaruddin J, Idzwan Z, Teo AH. Correlations between capnographic waveforms and peak flow meter measurement in emergency department management of asthma. Int J Emerg Med 2009;2:83-9.

58. Blanch L, Lucangelo U, Lopez-Aguilar J, Fernandez R, Romero PV. Volumetric capnography in patients with acute lung injury: effects of positive end-expiratory pressure. Eur Respir J 1999;13:1048-54.

59. Blanch L, Fernandez R, Saura P, Baigorri F, Artigas A. Relationship between expired capnogram and respiratory system resistance in critically ill patients during total ventilatory support. Chest 1994;105:219-23.

60. Babik B, Asztalos T, Petak F, Deak ZI, Hantos Z. Changes in respiratory mechanics during cardiac surgery. Anesth Analg 2003;96:1280-7, table of contents.

61. Hantos Z, Daroczy B, Suki B, Nagy S, Fredberg JJ. Input impedance and peripheral inhomogeneity of dog lungs. J Appl Physiol (1985) 1992;72:168-78.

62. Tusman G, Sipmann FS, Bohm SH. Rationale of dead space measurement by volumetric capnography. Anesth Analg 2012;114:866-74.

63. Ream RS, Schreiner MS, Neff JD, McRae KM, Jawad AF, Scherer PW, Neufeld GR. Volumetric capnography in children. Influence of growth on the alveolar plateau slope. Anesthesiology 1995;82:64-73.

64. Tsoukias NM, Tannous Z, Wilson AF, George SC. Single-exhalation profiles of NO and CO2 in humans: effect of dynamically changing flow rate. J Appl Physiol (1985) $1998 ; 85: 642-52$. 
65. Ioan I, Demoulin B, Duvivier C, Leblanc AL, Bonabel C, Marchal F, Schweitzer C, Varechova S. Frequency dependence of capnography in anesthetized rabbits. Respir Physiol Neurobiol 2014;190:14-9.

66. Fowler WS. Lung function studies; the respiratory dead space. Am J Physiol 1948;154:405-16.

67. Fowler WS. Respiratory dead space. Fed Proc 1948;7:35.

68. Breen PH, Mazumdar B, Skinner SC. Comparison of end-tidal PCO2 and average alveolar expired PCO2 during positive end-expiratory pressure. Anesth Analg 1996;82:368-73.

69. Singh BS, Gilbert U, Singh S, Govindaswami B. Sidestream microstream end tidal carbon dioxide measurements and blood gas correlations in neonatal intensive care unit. Pediatr Pulmonol 2013;48:250-6.

70. Steiger JH. Tests for comparing elements of a correlation matrix. Psychological Bulletin 1980;87:245-51.

71. Dellaca RL, Zannin E, Sancini G, Rivolta I, Leone BE, Pedotti A, Miserocchi G. Changes in the mechanical properties of the respiratory system during the development of interstitial lung edema. Respir Res 2008;9:51.

72. Schwardt JD, Neufeld GR, Baumgardner JE, Scherer PW. Noninvasive recovery of acinar anatomic information from CO2 expirograms. Ann Biomed Eng 1994;22:293306.

73. Tenling A, Hachenberg T, Tyden H, Wegenius G, Hedenstierna G. Atelectasis and gas exchange after cardiac surgery. Anesthesiology 1998;89:371-8.

74. Verheij J, van Lingen A, Raijmakers PG, Spijkstra JJ, Girbes AR, Jansen EK, van den Berg FG, Groeneveld AB. Pulmonary abnormalities after cardiac surgery are better explained by atelectasis than by increased permeability oedema. Acta Anaesthesiol Scand 2005;49:1302-10.

75. Babik B, Petak F, Asztalos T, Deak ZI, Bogats G, Hantos Z. Components of respiratory resistance monitored in mechanically ventilated patients. Eur Respir J 2002;20:1538-44.

76. van Meerten RJ. Expiratory gas concentration curves for examination of uneven distribution of ventilation and perfusion in the lung. First communication: theory. Respiration 1970;27:552-64. 
77. Albu G, Babik B, Kesmarky K, Balazs M, Hantos Z, Petak F. Changes in airway and respiratory tissue mechanics after cardiac surgery. Ann Thorac Surg 2010;89:121826.

78. Tolnai J, Szabari MV, Albu G, Maar BA, Parameswaran H, Bartolak-Suki E, Suki B, Hantos Z. Functional and morphological assessment of early impairment of airway function in a rat model of emphysema. J Appl Physiol (1985) 2012;112:19329.

79. Lorx A, Szabo B, Hercsuth M, Penzes I, Hantos Z. Low-frequency assessment of airway and tissue mechanics in ventilated COPD patients. J Appl Physiol (1985) 2009; 107:1884-92.

80. Lutchen KR, Hantos Z, Petak F, Adamicza A, Suki B. Airway inhomogeneities contribute to apparent lung tissue mechanics during constriction. J Appl Physiol (1985) 1996;80:1841-9.

81. Napolitano LM. Capnography in critical care: accurate assessment of ARDS therapy? Crit Care Med 1999;27:862-3.

82. Morley TF, Giaimo J, Maroszan E, Bermingham J, Gordon R, Griesback R, Zappasodi SJ, Giudice JC. Use of capnography for assessment of the adequacy of alveolar ventilation during weaning from mechanical ventilation. Am Rev Respir Dis 1993;148:339-44.

83. Tusman G, Bohm SH, Suarez-Sipmann F, Scandurra A, Hedenstierna G. Lung recruitment and positive end-expiratory pressure have different effects on $\mathrm{CO} 2$ elimination in healthy and sick lungs. Anesth Analg 2010;111:968-77. 
I. Csorba Z, Petak F, Nevery K, Tolnai J, Balogh AL, Rarosi F, Fodor GH, Babik B. Capnographic parameters in ventilated patients: correspondence with airway and lung tissue mechanics. Anesth \& Analg (Accepted for publication) [IF: 3.472] 


\section{CAPNOGRAPHIC PARAMETERS IN VENTILATED PATIENTS: CORRESPONDENCE WITH AIRWAY AND LUNG TISSUE MECHANICS}

\section{Zsofia Csorba, MD}

Affiliation: Department of Anesthesiology and Intensive Therapy, University of Szeged, Hungary

Email: drcsorbazsofia@gmail.com

Role: This author helped conduct the study and analyze the data

Conflicts: Zsofia Csorba reported no conflicts of interest

Attestation: Zsofia Csorba has seen the original study data, reviewed the analysis of the data, and approved the final manuscript

\section{Ferenc Petak, PhD}

Affiliation: Department of Medical Physics and Informatics, University of Szeged, Hungary

Email: petak.ferenc@med.u-szeged.hu

Role: This author helped design the study, conduct the study, analyze the data, and write the manuscript

Conflicts: Ferenc Petak reported no conflicts of interest

Attestation: Ferenc Petak has seen the original study data, reviewed the analysis of the data, and approved the final manuscript, and is the author responsible for archiving the study files

\section{Kitti Nevery}

Affiliation: Department of Anesthesiology and Intensive Therapy, University of Szeged, Hungary

Email: neverykitti@gmail.com

Role: This author helped conduct the study and analyze the data

Conflicts: Kitti Nevery reported no conflicts of interest

Attestation: Kitti Nevery has seen the original study data and approved the final manuscript

\section{Jozsef Tolnai, PhD}

Affiliation: Department of Medical Physics and Informatics, University of Szeged, Hungary

Email: tolnai.jozsef@med.u-szeged.hu

Role: This author helped conduct the study and analyze the data

Conflicts: Jozsef Tolnai reported no conflicts of interest

Attestation: Jozsef Tolnai has seen the original study data and approved the final manuscript

\section{Adam L. Balogh, MD}

Affiliation: Department of Anesthesiology and Intensive Therapy, University of Szeged, Hungary

Email: balogh.adam.laszlo@med.u-szeged.hu

Role: This author helped conduct the study and analyze the data

Conflicts: Adam L. Balogh reported no conflicts of interest

Attestation: Adam L. Balogh has seen the original study data and approved the final manuscript 


\section{Ferenc Rarosi}

Affiliation: Department of Medical Physics and Informatics, University of Szeged, Hungary

Email: rarosi.ferenc@med.u-szeged.hu

Role: This author helped analyze the data

Conflicts: Ferenc Rarosi reported no conflicts of interest

Attestation: Ferenc Rarosi has seen the original study data and approved the final manuscript

\section{Gergely H Fodor, MD}

Affiliation: Department of Medical Physics and Informatics, University of Szeged, Hungary

Email: fodor.gergely@med.u-szeged.hu

Role: This author helped analyze the data

Conflicts: Gergely H Fodor reported no conflicts of interest

Attestation: Gergely H Fodor has seen the original study data and approved the final manuscript

\section{Barna Babik, MD, PhD}

Affiliation: Department of Anesthesiology and Intensive Therapy, University of Szeged, Hungary

Email: babikbarna@gmail.com

Role: This author helped design the study, conduct the study, analyze the data, and write the manuscript

Conflicts: Barna Babik reported no conflicts of interest

Attestation: Barna Babik has seen the original study data, reviewed the analysis of the data, and approved the final manuscript

Institution: Department of Anesthesiology and Intensive Therapy, Department of Medical Physics and Informatics, University of Szeged

Short Title: Capnography in ventilated patients

Funding: Funded by a Hungarian Basic Research Grant (OTKA K81179). This research was supported by the European Union and the State of Hungary, co-financed by the European Social Fund in the framework of TÁMOP 4.2.4. A/2-11-1-2012-0001 'National Excellence Program'.

\section{Corresponding Author:}

Ferenc Petak, PhD

Department of Medical Physics and Informatics

Korányi fasor 9, Hungary H-6720

Phone: +36 62545077

FAX: +36 62545077

Email: petak.ferenc@med.u-szeged.hu

\section{Submitted as a Research Report}

This report describes human research. IRB contact information: Regional and 
Institutional Human Medical Biological Research Ethics Committee H-6720 Szeged, Korányi fasor 8-10.

Tel. +36 62 545997, e-mail: kutetika@gmail.com

\section{ABSTRACT}

Background: Although the mechanical status of the lungs affects the shape of the capnogram, the relationships between the capnographic parameters and those reflecting the airway and lung tissue mechanics have not been established in mechanically ventilated patients. We therefore set out to characterize how the mechanical properties of the airways and lung tissues modify the indices obtained from the different phases of the time and volumetric capnograms, and how the lung mechanical changes are reflected in the altered capnographic parameters after a cardiopulmonary bypass (CPB).

Methods: Anesthetized, mechanically ventilated patients $(n=101)$ undergoing heart surgery were studied in a prospective consecutive cross sectional study under the open-chest condition before and $5 \mathrm{~min}$ after CPB. Forced oscillation technique was applied to measure airway resistance (Raw), tissue damping $(\mathrm{G})$ and elastance $(\mathrm{H})$. Time and volumetric capnography were performed to assess parameters reflecting the phase II ( $\mathrm{S}_{\text {II }}$ ) and III slopes $\left(\mathrm{S}_{\mathrm{III}}\right)$, their transition $\left(\mathrm{D}_{2 \mathrm{~min}}\right)$, the dead-space indices according to Fowler, Bohr and Enghoff and the intrapulmonary shunt.

Results: Before $\mathrm{CPB}, \mathrm{S}_{\mathrm{II}}$ and $\mathrm{D}_{2 \min }$ exhibited the closest ( $\left.\mathrm{p}=0.006\right)$ associations with $\mathrm{H}(0.65$ and $-0.57 ; \mathrm{p}<0.0001$, respectively), whereas $S_{\text {III }}$ correlated most strongly $(\mathrm{p}<0.0001)$ with Raw $(\mathrm{r}=0.63 ; \mathrm{p}<0.0001)$. CPB induced significant elevations in Raw and $\mathrm{G}$ and $\mathrm{H}$ $(\mathrm{p}<0.0001)$. These adverse mechanical changes were reflected consistently in $\mathrm{S}_{\text {III }}, \mathrm{S}_{\mathrm{III}}$ and $\mathrm{D}_{2 \min }$, with weaker correlations with the dead-space indices $(\mathrm{p}<0.0001)$. The intrapulmonary shunt expressed as the difference between the Enghoff and Bohr dead-space parameters was increased after CPB $(95 \pm 5[\mathrm{SEM}] \%$ vs. $143 \pm 6 \%$; $<0.001)$.

Conclusions: In mechanically ventilated patients, the capnographic parameters from the early phase of expiration ( $S_{\text {II }}$ and $D_{2 \min }$ ) are linked to the pulmonary elastic recoil, while the effect of airway patency on $S_{\text {III }}$ dominates over the lung tissue stiffness. However, severe deteriorations in lung resistance or elastance affects both capnogram slopes.

Word count: 294

\section{INTRODUCTION}

The capnogram is a curve reflecting the concentration change of carbon dioxide $\left(\mathrm{CO}_{2}\right)$ as an endogenous indicator during expiration. In addition to verifying the correctness of the airway management, capnography provides information about the uniformity of lung emptying and adverse changes in the overall airway geometry (1-8) and respiratory tissue stiffness (6-8), and it serves as a valuable tool for the recognition of pulmonary circulatory abnormalities (9-11). International recommendations for standards require the monitoring of ventilation with capnography in all patients undergoing sedation or general anesthesia $(12,13)$.

Characterization of the relationships between standard lung function parameters and capnographic indices provides a valuable tool facilitating an understanding of the various shapes of the capnogram $(1,2,14)$. However, the earlier studies demonstrating associations between the capnographic slope factors with the forced expiratory volume in $1 \mathrm{~s}\left(\mathrm{FEV}_{1}\right)$ $(1,14)$ and the peak expiratory flow $(2,14)$ were limited to spontaneously breathing subjects. Despite the particular importance of recognizing adverse alterations in the pulmonary system in mechanically ventilated patients, details as to how the resistive and/or elastic properties of the pulmonary system affect the various indices derived from the capnogram are essentially lacking from the literature. In the present study, therefore, we set out to establish 
the connections between the various phase, shape, dead-space or pulmonary shunt circulation parameters of the time or volumetric capnogram and those reflecting the airway and lung tissue mechanics, expiratory flow and gas exchange. In order to gain an insight into within-subject alterations in the pulmonary condition, a large cohort of ventilated patients were examined during cardiac surgery, a cardiopulmonary bypass (CPB) being applied to generate a temporary complex realignment in the pulmonary mechanics and circulation.

\section{METHODS}

Detailed description of the patients' characteristics, methodology and results can be found in an online supplement.

\section{Patients}

After they have provided their written informed consent, 101 patients (female/male: 30/71, $62 \pm 9 \mathrm{yrs})$ undergoing elective open heart surgeries were examined in a prospective, consecutive cross sectional manner. The study protocol was approved by the Human Research Ethics Committee of Szeged University, Hungary (no. WHO 2788). Patients were excluded in the event of severe cardiopulmonary disorders (pleural effusion $>300 \mathrm{ml}$, ejection fraction $<30 \%, \mathrm{BMI}>35 \mathrm{~kg} / \mathrm{m}^{2}$ or intraoperative acute asthma exacerbation).

\section{Anesthesia and surgery}

Intravenous midazolam (30 $\mu \mathrm{g} / \mathrm{kg})$, sufentanil $(0.4-0.5 \mu \mathrm{g} / \mathrm{kg})$ and propofol $(0.3-0.5 \mathrm{mg} / \mathrm{kg}$ ) were applied to induce anesthesia. The maintenance of anesthesia and muscle relaxation was ensured by an intravenous infusion of propofol $(50 \mu \mathrm{g} / \mathrm{kg} / \mathrm{min})$ and intravenous boluses of rocuronium $(0.2 \mathrm{mg} / \mathrm{kg}$ every $30 \mathrm{~min})$.

Endotracheal intubation was performed and the patients were mechanically ventilated in volume-controled mode with descending flow (Dräger Zeus, Lübeck, Germany). The tidal volume was set to $7 \mathrm{ml} / \mathrm{kg}$ with a ventilator frequency of $9-14$ breaths $/ \mathrm{min}$, a positive endexpiratory pressure (PEEP) of $4 \mathrm{cmH}_{2} \mathrm{O}$, and an inspired oxygen fraction of $\left(\mathrm{FiO}_{2}\right)$ of 0.5 . Arterial blood gas samples were analyzed to calculate the Horowitz coefficient $\left(\mathrm{HQ}=\mathrm{P}_{\mathrm{aO}_{2}} / \mathrm{FiO}_{2}\right)$. During cardioplegic cardiac arrest, the lungs were not ventilated with maintaining no positive airway pressure. The lungs were then inflated 3-5 times to a peak airway pressure of $30 \mathrm{cmH}_{2} \mathrm{O}$ before declamping of the aorta to perform lung recruitment.

\section{Forced oscillatory measurements}

The low-frequency forced oscillation technique was applied to measure the lung mechanical properties, as detailed previously (15). The volume history was standardized by inflating the lungs to a pressure of $30 \mathrm{cmH}_{2} \mathrm{O}$ before the oscillatory measurements. Forced oscillatory signal was introduced into the lungs during short (15-s) apneic periods. The input impedance of the lung (ZL) was computed from the power spectra of the airway opening pressure and tracheal airflow. A model (16) containing a frequency-independent airway resistance (Raw) and inertance (Iaw) and a constant-phase tissue compartment characterized by the coefficients of damping $(\mathrm{G})$ and elastance $(\mathrm{H})$ was fitted to the mean ZL data. The lung tissue resistance (Rti) and the total lung resistance $(\mathrm{RL})$ at the ventilation frequency $(0.2 \mathrm{~Hz})$ was also calculated from the ZL spectra.

\section{Recording and analyses of the expiratory capnogram}

A mainstream capnograph (Novametrix, Capnogard ${ }^{\circledR}$, Andover, MA, USA) and another central airflow meter (Piston Ltd., Budapest, Hungary) were connected into the ventilatory circuit at the Y-piece, and 15-s $\mathrm{CO}_{2}$ and ventilator flow traces were recorded simultaneously. 
The $\mathrm{CO}_{2}$ and ventilator flow traces were digitized and imported into custom-made signal analysis software. The slopes of phase III of the capnogram in the time $\left(\mathrm{S}_{\mathrm{IIIT}}\right)$ and in the volumetric $\left(\mathrm{S}_{\mathrm{III}, \mathrm{V}}\right)$ domains were determined by fitting a linear regression line to the last twothirds of each phase-III traces (Fig. 1) $(17,18)$. The phase-II slopes of the time $\left(\mathrm{S}_{\mathrm{II}, \mathrm{T}}\right)$ and volumetric $\left(\mathrm{S}_{\mathrm{II}, \mathrm{V}}\right)$ capnograms were determined by calculating the slopes of the best-fitting line around the inflection point $( \pm 20 \%)$. Each slope was divided by the average corresponding $\mathrm{CO}_{2}$ concentration in the mixed expired gas to obtain normalized time ( $\mathrm{S}_{\mathrm{nII}, \mathrm{T}}$

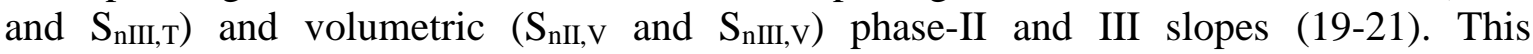
normalization was made only for the slope indices, as performed earlier before and after CPB (11). The angle ( $\alpha_{\text {cap }}$ ) formed by the phase-II and III limbs of the expiratory time capnogram was also calculated by using a standard monitoring speed of $12.5 \mathrm{mmHg} / \mathrm{s}$. The transition rates of change from phase II to phase III in the time $\left(\mathrm{D}_{2 \mathrm{~min}}\right)$ and volumetric $\left(\mathrm{D}_{2 \mathrm{Vmin}}\right)$ capnograms reflecting the curvature were calculated as the minima of the secondorder time and volumetric derivatives (22).

Besides these shape factors, dead-space parameters were derived from the volumetric capnograms. The Fowler dead space $\left(\mathrm{V}_{\mathrm{DF}}\right)$, reflecting the anatomic dead-space volume of the conducting airways was determined by calculating the expired gas volume until the inflection point of phase II was reached in the volumetric capnogram $(23,24)$. The physiological dead space, including also the alveolar volume not involved in gas exchange, was assessed by the Bohr method ( $\left.\mathrm{V}_{\mathrm{DB}}\right)$ (25). The dead space according to Enghoff's modification $\left(\mathrm{V}_{\mathrm{DE}}\right)$ was calculated; this takes also into account the ventilated, but not perfused alveoli (26).

We also calculated the differences between the Enghoff and Bohr dead-space parameters $\left(V_{D E}-V_{D B}\right)$ representing the pulmonary shunt circulation. The intrapulmonary shunt blood flow (Qs/Qt) was additionally assessed via the Fick equation.

Under both experimental conditions, 3 to 5 expiratory traces in each recording were analyzed, resulting in the ensemble-averaging of 10-12 values for further analysis in each patient.

\section{Analysis of the expiratory flow}

To characterize the expiratory flow pattern, the expiratory phases of each $\mathrm{V}$ ' recordings were analyzed by fitting an exponential function to the elevating limb (27):

$V^{\prime}(t)=V^{\prime}{ }_{p l}-P F \cdot e^{-L F \cdot t}$

where $\mathrm{V}^{\prime}$ pl is the plateau flow before the beginning of the next inspiration, $\mathrm{PF}$ is the peak expiratory flow, and LF is related to the curvature of the expiratory curve. The parameter LF is related to the curvature of the expiratory curve; a larger value indicates a more concave shape in the late flow. Model fitting to the serial data points from the peak flow was performed until $90 \%$ of the equilibrium value of $V^{\prime}(t)$ was reached.

\section{Measurement protocol}

Two sets of measurements were made under the open-chest condition $5 \mathrm{~min}$ before the CPB and 5 min after the patient was weaned from the CPB. Recruitment maneuvers were performed before the weaning from the CPB. Each data collection period started with recordings of 3 to 5 capnogram traces. During this period, an arterial blood gas sample was 
taken to measure $\mathrm{P}_{\mathrm{aO}}$ and $\mathrm{PaCO}_{2}$ for the calculation of $\mathrm{HQ}$ and $\mathrm{V}_{\mathrm{DE}}$, respectively. The total lung resistance $\left(\mathrm{R}_{\mathrm{vent}}\right)$ and compliance $\left(\mathrm{C}_{\mathrm{vent}}\right)$ displayed by the respiratory monitor of the ventilator were registered at this stage of the protocol. The data collections under both conditions were supplemented by recordings of 3 to $5 \mathrm{ZL}$ data epochs at 1 -min intervals.

\section{Data analyses}

Sample size estimation was applied to involve sufficient number of patients for the detection of clinically relevant significances. The type 1 error rate was set to 0.05 , the statistical power was set to 0.85 and the clinically relevant effect size (alternative hypothesis) was considered to detect correlation coefficients $r=0.3$ versus $r=0$. The necessary sample size was 96 .

Scatters in measured variables are expressed as SEM values. In the event of passing the normality test (marked in footnotes), paired t-tests were used to examine the statistical significance of the changes induced in the parameters by the CPB. Wilcoxon signed-rank tests were applied otherwise to verify the significance of the changes in the mechanical, capnographic or gas exchange parameters. The Pearson test was applied to analyze the correlations between the different variables. The comparison of Pearson correlation coefficients were made by Steiger's Z-test; these tests were performed between the particular and the nearest $r$ values. Subgroups of patients were formed based on the initial HQ level (high and low 25 percentile), and based on the extremity of changes after surgery (top 25 percentile increase and bottom 25 percentile decrease in $\mathrm{HQ}$, respectively). Time domain capnogram slope indices and Raw and $\mathrm{C}_{\mathrm{vent}}$ and their changes after the surgery were also correlated in these subgroups and were compared to the results obtained from the pooled population. Values $\mathrm{p}<0.05$ were considered to be statistically significant.

\section{RESULTS}

Parameters reflecting the lung mechanics and the expiratory flow are demonstrated in Fig. 2. All the resistive parameters including those reflecting the flow resistance of the airways (Raw) or of the lung tissues (Rti) or the combination of these compartments ( $\mathrm{R}_{\text {vent }}$ and $\mathrm{RL}$ ), exhibited marked and statistically significant increases after CPB ( $<0.0001$ for each). Conversely, more moderate, but still highly significant decreases were observed following $\mathrm{CPB}$ in the compliance parameters determined at end-expiratory lung volume by the oscillometry $(\mathrm{CL})$ or at end-inspiratory lung volume by the ventilator $\left(\mathrm{C}_{\mathrm{vent}}\right)(\mathrm{p}<0.0001$ for both). CPB induced no statistically detectable changes in $\mathrm{PF}(\mathrm{p}=0.5)$, whereas the parameter $\mathrm{LF}$, reflecting the curvature of the late flow, increased significantly $(\mathrm{p}<0.0001)$. The CPBinduced adverse lung mechanical changes were also reflected in the significant decrease in HQ (from $371 \pm 11$ to $350 \pm 14 \mathrm{mmHg}$; $=0.038^{3}$ ).

Figure 3 depicts the indices derived from the time and volumetric capnographic measurements before and after the CPB. Marked and statistically significant increases were observed in the time and volumetric parameters reflecting the phase-III slope of the expired $\mathrm{CO}_{2}\left(\mathrm{p}<0.0001\right.$ for $\mathrm{S}_{\text {III,T, }}, \mathrm{S}_{\mathrm{nIII}, \mathrm{T}}, \mathrm{S}_{\mathrm{III}, \mathrm{V}}$ and $\left.\mathrm{S}_{\mathrm{nIII}, \mathrm{V}}\right)$ after the CPB. The slopes of phase II revealed significant decreases following CPB ( $p<0.0001$ for both $S_{\text {II,T }}$ and $S_{I I, V}$ ), whereas these drops were no longer detectable after normalization to the $\mathrm{CO}_{2}$ concentration in the mixed expired gas $\left(\mathrm{p}=0.4\right.$ and 0.9 for $\mathrm{S}_{\mathrm{nII}, \mathrm{T}}$ and $\mathrm{S}_{\mathrm{nII}, \mathrm{V}}$, respectively $\left.{ }^{1}\right)$. CPB increased the curvature representing the transition from phase II to phase III ( $<<0.0001$ for both $\mathrm{D}_{2 \mathrm{~min}}$ and $\left.\mathrm{D}_{2 \text { Vmin }}\right)$. Uniform decreases were detected in $\mathrm{V}_{\mathrm{DF}}$ and $\mathrm{V}_{\mathrm{DB}}(\mathrm{p}<0.0001)$ after the $\mathrm{CPB}$, whereas

\footnotetext{
${ }^{3}$ Shapiro-Wilk test for normality passed $(\mathrm{p}>0.41)$
} 
$\mathrm{V}_{\mathrm{DE}}$ increased significantly $(\mathrm{p}<0.0001)$. These changes in the dead space parameters resulted in significant elevations in the shunt parameters reflecting the alterations in lung ventilation $\left(\mathrm{p}=0.02\right.$ and $\mathrm{p}<0.0001$ for $\mathrm{V}_{\mathrm{DB}}-\mathrm{V}_{\mathrm{DF}}$ and $\mathrm{V}_{\mathrm{DE}}-\mathrm{V}_{\mathrm{DB}}$, respectively ${ }^{4}$ ) and perfusion (Qs/Qt, $\mathrm{p}<0.0001)$.

Figure 4 illustrates the strengths of the correlations between the lung mechanical parameters (x-axis) and the time and volumetric capnographic parameters reflecting the slopes, transitions, dead-space and shunt fractions (y-axis).

The lung resistive parameters exhibited the closest associations with the phase-III slope capnographic parameters $(\mathrm{p}<0.0001)$, particularly after the $\mathrm{CPB}$, when all the indices reflecting the resistive properties of the pulmonary system were markedly elevated ( $<<0.0001$; Fig. 4, top panels). Significant, but somewhat weaker correlations were observed between the lung resistive parameters and the ventilation dead-space parameters $V_{D F}$ $(\mathrm{p}<0.0001)$ and $\mathrm{V}_{\mathrm{DB}}(\mathrm{p}<0.0001)$. More specifically, the mechanical parameter representing the flow resistance of the airways (Raw) correlated best $(\mathrm{p}<0.0001)$ with the $\mathrm{S}_{\text {III,T }}(\mathrm{r}=0.63$ and 0.68 for $\mathrm{S}_{\mathrm{III}, \mathrm{T}}$ before and after the $\mathrm{CPB}$, respectively; $\left.\mathrm{p}<0.0001\right)$. Moreover, Raw correlated significantly with $\mathrm{S}_{\text {III,V }}\left(\mathrm{r}=0.43\right.$ and 0.55 for $\mathrm{S}_{\mathrm{III}, \mathrm{V}}$ before and after CPB, respectively, $\mathrm{p}<0.0001)$. Conversely, the mechanical parameters characterizing lung tissue elasticity $\left(\mathrm{H}\right.$ and $\left.\mathrm{C}_{\mathrm{vent}}\right)$ showed the closest $(\mathrm{p}=0.006)$ relationships with the time capnographic parameters describing the phase II ( $\mathrm{r}=0.65$ and 0.41 between $\mathrm{H}$ and $\mathrm{S}_{\mathrm{II}, \mathrm{T}}$ before and after the CPB, respectively; $\mathrm{p}<0.0001$ ). Normalization of the phase-III slopes to the $\mathrm{CO}_{2}$ concentration in the mixed expired gas did not affect these relationships noticeably $(\mathrm{p}=0.71)$. The pulmonary elastance and compliance parameters also revealed close associations with the capnographic indices reflecting the curvatures of the transitions between the phases, particularly before the CPB ( $r=-0.57$ between $\mathrm{H}$ and $\left.\mathrm{D}_{2 \mathrm{~min}} ; \mathrm{p}<0.0001\right)$. The early and latephase expiratory flow parameters revealed strong associations between $\mathrm{PF}$ and the deadspace indices both before and after the CPB. LF exhibited the strongest correlation with $\mathrm{S}_{\mathrm{nIII}, \mathrm{V}}(\mathrm{r}=0.53 ; \mathrm{p}<0.0001)$.

As concerns the relationships between the CPB-induced changes in the lung mechanical and capnographic indices (Fig. 4, bottom panel), the marked elevations in Raw correlated best $(\mathrm{p}=0.001)$ with the decreases in the phase-II slope parameters of the time capnogram $(\mathrm{r}=-0.72$

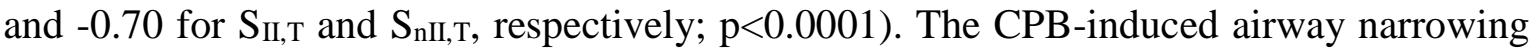
was also reflected in the elevated phase-III slope parameters of the time and volumetric capnograms ( $r=0.49$ for both $S_{\text {III,T }}$ and $\left.S_{\text {III,V; }} p<0.0001\right)$, and the curvature of the transition between the phases in the time domain $\left(r=0.6\right.$ for $\left.\mathrm{D}_{2 \mathrm{~min}} ; \mathrm{p}<0.0001\right)$. The changes in the other mechanical parameters reflecting the tissue $(\mathrm{G})$ or total lung resistance (RL or $\mathrm{R}_{\mathrm{vent}}$ ) displayed similar relationships with the alterations in the various capnographic indices after the CPB. Assessment of the mild CPB-induced stiffening of the lung tissue also revealed statistically significant correlations between the changes in $\mathrm{C}_{\mathrm{vent}}$ and those in the phase-III slope parameters in both the time and volumetric capnograms ( $\mathrm{r}=-0.48$ for both $\mathrm{S}_{\mathrm{nIII}, \mathrm{T}}$ and $\left.\mathrm{S}_{\text {nIII,V }} ; \mathrm{p}<0.0001\right)$. Neither the absolute values of HQ nor the changes following CPB exhibited close relationships with any other mechanical or capnographic indices; an association was observed with $\mathrm{V}_{\mathrm{DE}}$ before the $\mathrm{CPB}(\mathrm{r}=0.31 ; \mathrm{p}<0.0002)$.

The relationships between the initial fundamental lung mechanical and capnographic indices for the subgroups of patients based on starting HQ are depicted on Fig. 5A. Strong positive significant correlations were observed between Raw and phase III slope parameters $(\mathrm{p}=0.002)$ and between $\mathrm{C}_{\mathrm{vent}}$ and phase II slope parameters independently of the subgroup

\footnotetext{
${ }^{4}$ Shapiro-Wilk test for normality passed $(\mathrm{p}>0.13)$
} 
allocation $(\mathrm{p}=0.001)$. The initial $\mathrm{C}_{\mathrm{vent}}-\mathrm{S}_{\mathrm{III}, \mathrm{T}}$ relationship was not significantly correlated $(\mathrm{p}=0.20)$, while the Raw-S $\mathrm{S}_{\text {II,T }}$ correlation appeared significant only for the pooled patient population ( $\mathrm{p}=0.0045)$. The changes in Raw correlated to those in both slope variables ( $\mathrm{p}<0.0001)$, whereas the alterations in $\mathrm{C}_{\text {vent }}$ were significantly related with those in phase III slopes ( $\mathrm{p}=0.023$, Fig. 5B).

Findings reflecting interindividual variability and demonstrating individual changes are included in the online supplement (Table 1S and Fig. 1S). A large range was obtained for the coefficient of variation in the initial lung mechanical and capnograpm parameters (ranging from $25 \%$ to $169 \%$ for $\mathrm{V}_{\mathrm{DB}}$ and $\mathrm{S}_{\mathrm{nIII}, \mathrm{T}}$, respectively). Further interdependences of the main capnogram shape factors and lung mechanical parameters are illustrated in Fig. $2 \mathrm{~S}$ in the online supplement.

DISCUSSION

Capnography is an essential part of the monitoring in patients requiring mechanical ventilation. The present study was motivated to elucidate how changes in the mechanical properties of the different lung compartments are reflected in the alterations in the shape, dead-space and pulmonary shunt circulation parameters obtained from the time or volumetric capnograms. A detailed characterization of the airway and lung tissue mechanics was combined with a comprehensive evaluation of the capnographic indices before and after a lung function deterioration induced by a CPB. Our study revealed the specific influence of the lung resistive and elastic parameters on the capnogram shape indices in ventilated patients.

Phase-III slope

The results demonstrated significant increases in both $S_{\text {III,T }}$ and $S_{\text {III,V }}$ immediately following the CPB. The elevations also appeared after normalization to the $\mathrm{CO}_{2}$ concentration in the mixed expired gas (11) (Fig. 3). This finding differs from that observed previously in a smaller cohort of ventilated cardiac surgery patients, where no major changes were observed in the phase-III slope after CPB (11). The discrepancy may be attributed to the more aggressive maneuvers applied to recruit the lungs after the CPB in this earlier study, to the application of a higher PEEP ( 7 vs. $4 \mathrm{cmH}_{2} \mathrm{O}$ ) and to the somewhat delayed measurement time after the CPB (15 min vs. 5 min).

The phase-III slope of the capnogram is considered to reflect the summation of the ventilation inhomogeneities relating to the working alveolar compartments with different time constants and the ventilation-perfusion mismatch as concerns the dead-space and/or intrapulmonary shunt. The overall and the regional lung emptying are determined by the opposite effects of Raw, G and the lung recoil tendencies (6). The role of the lung tissue stiffness decreases dynamically toward the end of expiration, and the elastic recoil affects $S_{\text {III }}$ in patients with low or high compliance (6). Raw, therefore, exerted the primary influence on the $S_{\text {III }}$ parameters before the CPB (Figs 4 and 1S), independent from the initial lung function (i.e. HQ; Fig. 5A). This finding is in accord with the postulate of the close link between the airway cross-sectional area and $\mathrm{S}_{\text {III }}$, based on spirometric data obtained previously in spontaneously breathing patients $(1,3,28)$. Representing $(\mathrm{G})$ or incorporating lung parenchymal resistive component ( $R L$ and $\mathrm{R}_{\mathrm{vent}}$ ) weakened the correlation substantially ( $\mathrm{p}<0.0001$; Fig. 4). This suggests that under baseline conditions the internal friction in the lung tissue does not exert a major effect on the capnographic $S_{\text {III }}$ indices, along with the lesser role of compliance. Following the CPB, significant associations appeared between the overall resistive and capnographic phase-III slope parameters, due to the greatly elevated tissue resistance (Fig. 4). Thus, an elevated $\mathrm{S}_{\text {III }}$ may indicate the presence of lung disorders affecting not only the airways, but also the tissue resistive properties, such as observed during interstitial edema in sepsis or cardiac failure (29). These phenomena are 
comprehensively confirmed by the significantly elevated concavity of the late expiratory flow (Fig. 2), the increase in $\mathrm{V}_{\mathrm{DE}}$ and the diminished HQ (Fig. 3).

Phase-II slope

The phase-II slope was decreased following the CPB in both the time and the volumetric capnograms. This agrees with the results of the only earlier study, where the changes in $S_{\text {II }}$ were measured $2 \mathrm{~min}$ after establishment of the full pulmonary blood flow (11). However, normalization of $S_{\text {II }}$ to a possible lower $\mathrm{CO}_{2}$ content of the expired gas (i.e. $S_{n I I}$ ) after weaning from the CPB eliminated these changes (Fig. 3), because the intensity of axial gas mixing depends on the $\mathrm{CO}_{2}$ concentration (11).

Phase II of the capnogram represents the overall width of the moving airway-alveolar gas front and its slope may be explained by opposing effects. The heterogeneous start of lung emptying, the reduced airway lumen and increased tissue damping may all contribute to the decreases, whereas an elevated elastic recoil and a low alveolar $\mathrm{CO}_{2}$ content may counteract these changes in $S_{\text {II }}(1,3,11,30)$. Before the CPB, the correlation analyses of the $S_{\text {II }}$ parameters indicated their close relationship with the elastic properties of the lungs (Fig. 4), independent from the starting gas exchange ability of the lungs (Fig. 5A). This finding is in accordance with a wider phase II observed previously in emphysematous patients $(31,32)$, and increases in $S_{\text {II }}$ after compliance elevation through recruitment maneuvers (30). Since $\mathrm{PF}$ is determined more by the lung tissue stiffness $(\mathrm{r}=0.34 ; \mathrm{p}<0.0001 \mathrm{for} \mathrm{H})$ than by the airway caliber ( $\mathrm{r}=0.09 ; \mathrm{p}=0.34$ for Raw), the significant correlation of PF with the phase-II capnographic indices may also be attributed to the influence of the lung elastance during early expiration.

Independent of the direction and magnitude of change in HQ, the CPB-induced changes in $\mathrm{S}_{\text {II }}$ exhibited close correlations with the markedly elevated Raw (Fig. 5B) and lung resistance parameters (RL and $\mathrm{R}_{\mathrm{vent}}$; Fig. 4). This finding indicates that inhomogeneous airway constriction leads to a more sequential emptying of lung compartments with different $\mathrm{CO}_{2}$ content even at the beginning of expiration, and thereby widens the airway-alveolar gas front with subsequent decreases in the phase-II slope. The loss of correlations between the changes in $\mathrm{S}_{\mathrm{II}, \mathrm{T}}$ and $\mathrm{C}_{\mathrm{vent}}$ (Fig. 5B) may be due to the complex and opposing phenomena affecting $\mathrm{S}_{\mathrm{II}, \mathrm{T}}$, as described earlier.

\section{Capnographic parameters reflecting phase transitions}

The transition indices $\left(\alpha_{\text {cap }}, \mathrm{D}_{2 \mathrm{~min}}\right.$ and $\left.\mathrm{D}_{2 \mathrm{Vmin}}\right)$ reside in the same part of the capnogram, but their meanings are different. $\alpha_{\text {cap }}$ is the angle between $S_{\text {II }}$ and $S_{\text {III }}$, i.e. the relationship between the overall gas front and the alveolar gas volume, while $\mathrm{D}_{2 \min }$ and $\mathrm{D}_{2 \mathrm{Vmin}}$ are related to the internal surface of the moving $\mathrm{CO}_{2}$ diffusion front in the airways during expiration. The elevation observed in $\alpha_{\text {cap }}$ after the CPB reflects the combined alterations in $\mathrm{S}_{\text {II }}$ and $\mathrm{S}_{\text {III }}$, whereas both second derivative parameters approached zero after the CPB (Fig. 3), demonstrating blunted (less cornered) transitions between capnographic phases II and III. This finding may be attributed to the highly heterogeneous severe airway constriction that developes after the CPB, which blurs the resulting diffusion front measured in the central airway. The close associations between $\mathrm{FEV}_{1}$ and the capnographic indices reflecting the phase-II to phase-III transition in spontaneously breathing patients is in accordance with this result (1). Our data further demonstrate that, in ventilated patients, the low compliance associated with the normal airway patency compresses the flow profile, resulting in a sharper phase-II to phase-III transition (Fig. $1 \mathrm{~S}, \mathrm{C}$ ). This finding reveals the sensitivity of $\mathrm{D}_{2 \mathrm{~min}}$ and $\mathrm{D}_{2 \mathrm{Vmin}}$ parameters to changes in lung compliance as opposed to $\alpha_{\text {cap }}$, which demonstrates rather resistive properties (Fig. 4). 
The anatomical dead space $\left(\mathrm{V}_{\mathrm{DF}}\right)$ was decreased slightly but consistently after the CPB (Fig. 3). Since this change was associated with marked increases in Raw and LF (Fig. 4, bottom), the compromised lumen of the conducting airways and/or their exclusion from the ventilation may explain this finding. The dead-space parameter incorporating the additional volume of the unperfused alveoli $\left(\mathrm{V}_{\mathrm{DB}}\right)$ followed very similar changing and correlation patterns, indicating the negligible unperfused, but ventilated alveolar compartment after the CPB. The bronchoconstriction resulting from the additive effects of SIRS and local hypocapnia may contribute to the low alveolar dead space. Conversely, supine position, surgery and CPB led to elevations in $\mathrm{V}_{\mathrm{DE}}$ (Fig. 3), suggesting a substantial enlargement of the volume of the not ventilated but perfused alveoli due to persistent atelectasis after the CPB $(33,34)$.

The difference between $\mathrm{V}_{\mathrm{DE}}$ and $\mathrm{V}_{\mathrm{DB}}$, which approximates the extent of the pulmonary shunt, was increased markedly after the CPB (Fig. 3). It is noteworthy that $V_{D E}-V_{D B}$ exhibited parallel changes and a significant correlation $(r=0.47 ; p<0.0001)$ with the shunt fraction obtained from the classical shunt equation (Qs/Qt), highlighting the additional usefulness of volumetric capnography in the assessment of the intrapulmonary shunt (Fig. $3)$.

\section{Methodological aspects}

While patients with severe cardiopulmonary disorders were excluded from the present study, the pulmonary status of the participating subjects varied widely from relatively healthy lungs to obstructive and restrictive disorders. Such interindividual variety of pulmonary symptoms with additional demographic and anthropometric differences are expected to occur in all health-care units providing ventilatory support. Therefore, this feature of the study is particularly favorable and also facilitates the performance of the correlation analyses.

It is also noteworthy that the results represent an open chest condition. Significant alteration in lung-thorax dynamics is expected to influence both the capnography indices and forced oscillatory data reflecting airway and tissue mechanics (35). The capnogram parameters are determined by the heterogeneity of the lungs, geometry of the airway tree and the forces exerted by the tissue resistive and elastic properties of the lungs and the chest wall (6). While our study allows an insight into the mechanisms coupling the capnogram and mechanical parameters, a further study in intact chest patients may be needed to generalize our findings. A further important methodological aspect of the present study is related to the use of correlation analyses to assess the associations between parameters obtained by two different techniques. As a general rule, the existence of significant correlations between variables is necessary, but not sufficient to imply a causal relationship. In the present study, the lung mechanical and capnographic parameters are linked to each other through local common mechanisms governing lung emptying. Furthermore, the individual correlation results from consistent physiological and clinical findings. These considerations verify that causation can be inferred with great certainty.

Summary and conclusions

In conclusion, we characterized the relationships between the time or volumetric capnographic parameters and the lung mechanical indices reflecting the airway and the lung tissue viscoelastic properties in cardiac surgery patient underwent open heart surgery. The lung tissue stiffness predominantly determines the capnographic parameters in the early phase of expiration, since the elastic forces are maximal at high lung volumes. Thus, in the vast majority of the cases, the phase-II slope of the capnogram is predominantly determined by pulmonary elastic recoil. Conversely, the resistive properties of the lungs become increasingly important during the later phase of expiration and thus, the phase-III slope is shaped overwhelmingly by the airway resistance. However, markedly elevated lung 
resistance additionally worsens the capnogram phase II slope. Similarly, severely compromised lung elastance also distorts the capnogram phase III slope. Since computational methods could be incorporated into the modern anesthesia machines to quantify capnographic shape factors, these parameters together with the traditional bedside mechanical indices has the promise to improve differential diagnoses and advance guiding respiratory therapy.

\section{REFERENCES}

1. You B, Peslin R, Duvivier C, Vu VD, Grilliat JP. Expiratory capnography in asthma: evaluation of various shape indices. The European respiratory journal 1994;7:31823.

2. Yaron M, Padyk P, Hutsinpiller M, Cairns CB. Utility of the expiratory capnogram in the assessment of bronchospasm. Annals of emergency medicine 1996;28:403-7.

3. Krauss B. Capnography as a rapid assessment and triage tool for chemical terrorism. Pediatric emergency care 2005;21:493-7.

4. Stromberg NO, Gustafsson PM. Ventilation inhomogeneity assessed by nitrogen washout and ventilation-perfusion mismatch by capnography in stable and induced airway obstruction. Pediatric pulmonology 2000;29:94-102.

5. Blanch L, Romero PV, Lucangelo U. Volumetric capnography in the mechanically ventilated patient. Minerva anestesiologica 2006;72:577-85.

6. Babik B, Csorba Z, Czovek D, Mayr PN, Bogats G, Petak F. Effects of respiratory mechanics on the capnogram phases: importance of dynamic compliance of the respiratory system. Critical care 2012;16:R177.

7. Romero PV, Lucangelo U, Lopez Aguilar J, Fernandez R, Blanch L. Physiologically based indices of volumetric capnography in patients receiving mechanical ventilation. The European respiratory journal 1997;10:1309-15.

8. Yang Y, Huang Y, Tang R, Chen Q, Hui X, Li Y, Yu Q, Zhao H, Qiu H. Optimization of positive end-expiratory pressure by volumetric capnography variables in lavageinduced acute lung injury. Respiration; international review of thoracic diseases 2014;87:75-83.

9. Chopin C, Fesard P, Mangalaboyi J, Lestavel P, Chambrin MC, Fourrier F, Rime A. Use of capnography in diagnosis of pulmonary embolism during acute respiratory failure of chronic obstructive pulmonary disease. Critical care medicine 1990;18:353-7.

10. Verschuren F, Liistro G, Coffeng R, Thys F, Roeseler J, Zech F, Reynaert M. Volumetric capnography as a screening test for pulmonary embolism in the emergency department. Chest 2004;125:841-50.

11. Tusman G, Areta M, Climente C, Plit R, Suarez-Sipmann F, Rodriguez-Nieto MJ, Peces-Barba G, Turchetto E, Bohm SH. Effect of pulmonary perfusion on the slopes of single-breath test of CO2. Journal of applied physiology 2005;99:650-5.

12. Merry AF, Cooper JB, Soyannwo O, Wilson IH, Eichhorn JH. International Standards for a Safe Practice of Anesthesia 2010. Canadian journal of anaesthesia $=$ Journal canadien d'anesthesie 2010;57:1027-34.

13. Guidelines for Patient Care in Anesthesiology. http://www.asahq.org/resources/standards-and-guidelines: American Society of Anesthsiologists, 2011.

14. Veronez L, Pereira MC, da Silva SM, Barcaui LA, De Capitani EM, Moreira MM, Paschoal IA. Volumetric capnography for the evaluation of chronic airways diseases. International journal of chronic obstructive pulmonary disease 2014;9:983-9. 
15. Babik B, Asztalos T, Petak F, Deak ZI, Hantos Z. Changes in respiratory mechanics during cardiac surgery. Anesthesia and analgesia 2003;96:1280-7, table of contents.

16. Hantos Z, Daroczy B, Suki B, Nagy S, Fredberg JJ. Input impedance and peripheral inhomogeneity of dog lungs. Journal of applied physiology 1992;72:168-78.

17. Krauss B, Deykin A, Lam A, Ryoo JJ, Hampton DR, Schmitt PW, Falk JL. Capnogram shape in obstructive lung disease. Anesth Analg 2005;100:884-8, table of contents.

18. Blanch L, Lucangelo U, Lopez-Aguilar J, Fernandez R, Romero PV. Volumetric capnography in patients with acute lung injury: effects of positive end-expiratory pressure. Eur Respir J 1999;13:1048-54.

19. Ream RS, Schreiner MS, Neff JD, McRae KM, Jawad AF, Scherer PW, Neufeld GR. Volumetric capnography in children. Influence of growth on the alveolar plateau slope. Anesthesiology 1995;82:64-73.

20. Tusman G, Areta M, Climente C, Plit R, Suarez-Sipmann F, Rodriguez-Nieto MJ, Peces-Barba G, Turchetto E, Bohm SH. Effect of pulmonary perfusion on the slopes of single-breath test of CO2. J Appl Physiol 2005;99:650-5.

21. Tsoukias NM, Tannous Z, Wilson AF, George SC. Single-exhalation profiles of NO and $\mathrm{CO} 2$ in humans: effect of dynamically changing flow rate. J Appl Physiol 1998;85:642-52.

22. Ioan I, Demoulin B, Duvivier C, Leblanc AL, Bonabel C, Marchal F, Schweitzer C, Varechova S. Frequency dependence of capnography in anesthetized rabbits. Respiratory physiology \& neurobiology 2014;190:14-9.

23. Fowler WS. Lung function studies; the respiratory dead space. The American journal of physiology 1948;154:405-16.

24. Fowler WS. Respiratory dead space. Federation proceedings 1948;7:35.

25. Bohr C. Über die Lungenatmung. Skan Arch Physiol 1891;53:236-8.

26. Enghoff H. Volumen inefficax. Uppsala Laekareforen Forh 1938;44:191-218.

27. van Drunen EJ, Chiew YS, Chase JG, Shaw GM, Lambermont B, Janssen N, Damanhuri NS, Desaive T. Expiratory model-based method to monitor ARDS disease state. Biomedical engineering online 2013;12:57.

28. Nik Hisamuddin NA, Rashidi A, Chew KS, Kamaruddin J, Idzwan Z, Teo AH. Correlations between capnographic waveforms and peak flow meter measurement in emergency department management of asthma. International journal of emergency medicine 2009;2:83-9.

29. Dellaca RL, Zannin E, Sancini G, Rivolta I, Leone BE, Pedotti A, Miserocchi G. Changes in the mechanical properties of the respiratory system during the development of interstitial lung edema. Respiratory research 2008;9:51.

30. Tusman G, Bohm SH, Suarez-Sipmann F, Turchetto E. Alveolar recruitment improves ventilatory efficiency of the lungs during anesthesia. Canadian journal of anaesthesia = Journal canadien d'anesthesie 2004;51:723-7.

31. Kars AH, Bogaard JM, Stijnen T, de Vries J, Verbraak AF, Hilvering C. Dead space and slope indices from the expiratory carbon dioxide tension-volume curve. The European respiratory journal 1997;10:1829-36.

32. Schwardt JD, Neufeld GR, Baumgardner JE, Scherer PW. Noninvasive recovery of acinar anatomic information from $\mathrm{CO} 2$ expirograms. Annals of biomedical engineering 1994;22:293-306.

33. Tenling A, Hachenberg T, Tyden H, Wegenius G, Hedenstierna G. Atelectasis and gas exchange after cardiac surgery. Anesthesiology 1998;89:371-8. 
34. Verheij J, van Lingen A, Raijmakers PG, Spijkstra JJ, Girbes AR, Jansen EK, van den Berg FG, Groeneveld AB. Pulmonary abnormalities after cardiac surgery are better explained by atelectasis than by increased permeability oedema. Acta anaesthesiologica Scandinavica 2005;49:1302-10.

35. Barnas GM, Campbell DN, Mackenzie CF, Mendham JE, Fahy BG, Runcie CJ, Mendham GE. Lung, chest wall, and total respiratory system resistances and elastances in the normal range of breathing. The American review of respiratory disease 1992;145:110-3.

FIGURES
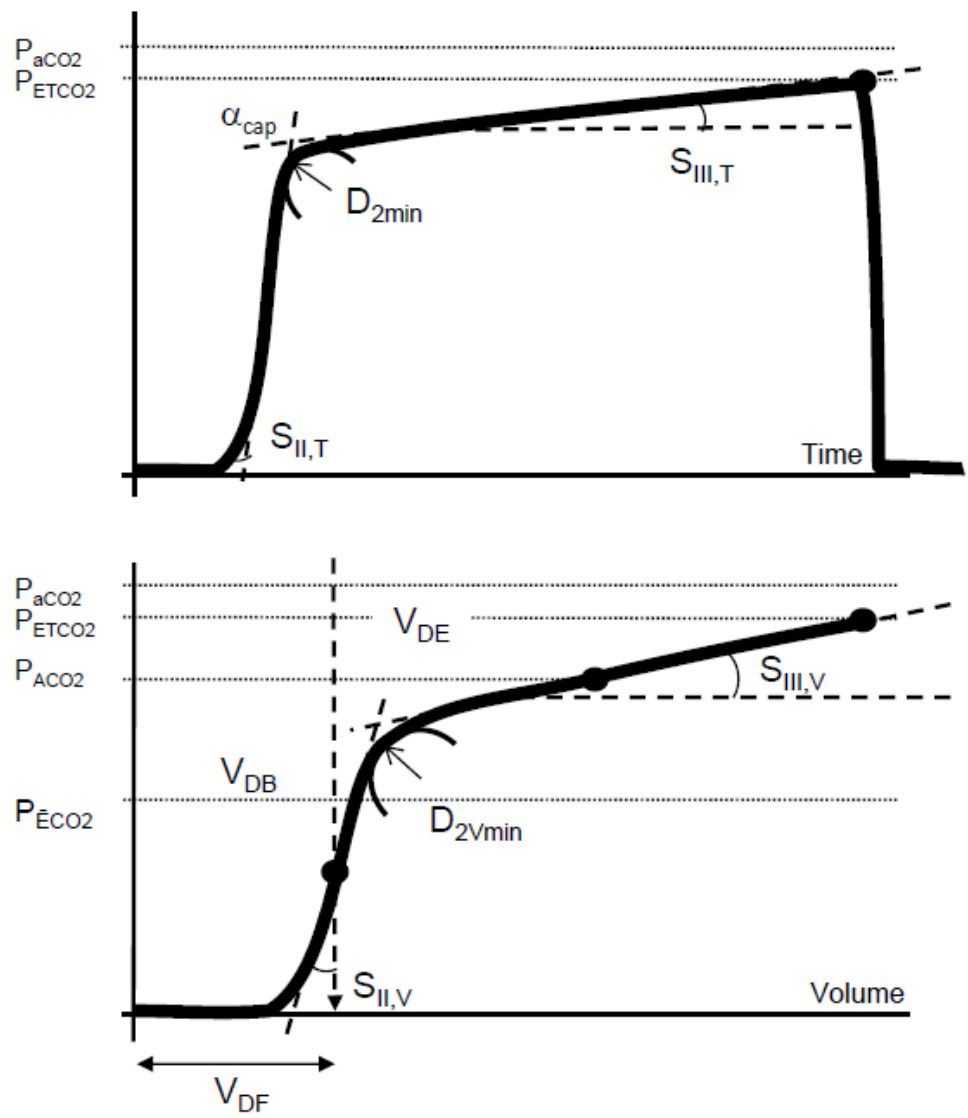

Figure 1. Shape factors and characteristic partial pressures derived from the time (top) and volumetric (bottom) capnograms. $\mathrm{P}_{\mathrm{aCO}_{2}}$ : partial pressure of arterial blood $\mathrm{CO}_{2} ; \mathrm{P}_{\mathrm{ACO}_{2}}$ : mean alveolar $\mathrm{CO}_{2}$ concentration at the midpoint of phase III of $\mathrm{CO}_{2}$ expiration; $\mathrm{P}_{\overline{\mathrm{E}} \mathrm{CO}_{2}}$ : mixed partial pressure of $\mathrm{CO}_{2}$ during the entire expiration; $\mathrm{P}_{\mathrm{ETCO}_{2}}$ : end-tidal $\mathrm{CO}_{2}$ concentration. $\mathrm{S}_{\text {II,T,V: }}$ phase II slope of the time and volumetric capnogram, respectively. SIII,T,V: phase III slope of the time and volumetric capnogram, respectively. $\mathrm{D}_{2 \min }$ and $\mathrm{D}_{2 \mathrm{Vmin}}$ : curvature at the phase II-III transitions, calculated as the minimum of the second-order time and volumetric derivative, respectively. $\alpha_{\text {cap }}$ : angle formed by the phase-II and phase-III limbs of the expiratory time capnogram. 

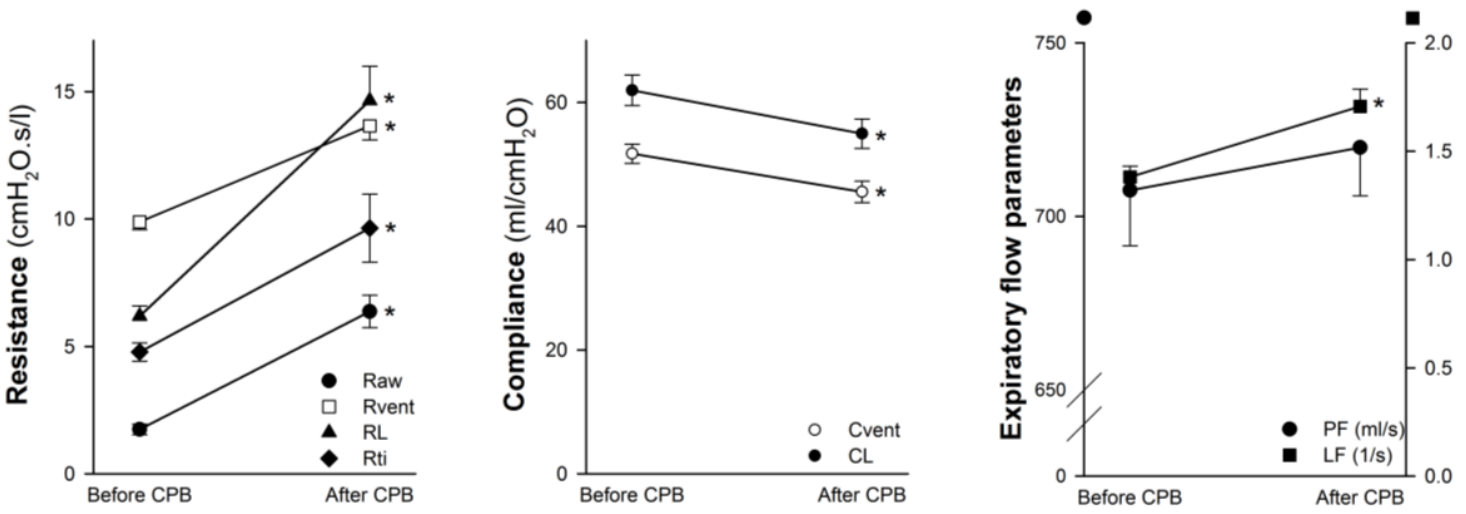

Figure 2. Resistive (Raw: airway resistance, $\mathrm{R}_{\mathrm{vent}}$ : total lung resistance displayed by the ventilator, RL: total lung resistance obtained by oscillometry, Rti: tissue resistance obtained by oscillometry) and elastic lung mechanical parameters $\left(\mathrm{C}_{\text {vent }}\right.$ : compliance displayed by the ventilator, CL: compliance determined by oscillometry) and expiratory flow indices (PF: peak flow, LF: late flow) before and after the cardiopulmonary bypass (CPB). *: $\mathrm{p}<0.05$ before vs. after the CPB. Values are expressed as mean $\pm \mathrm{SEM}$. 

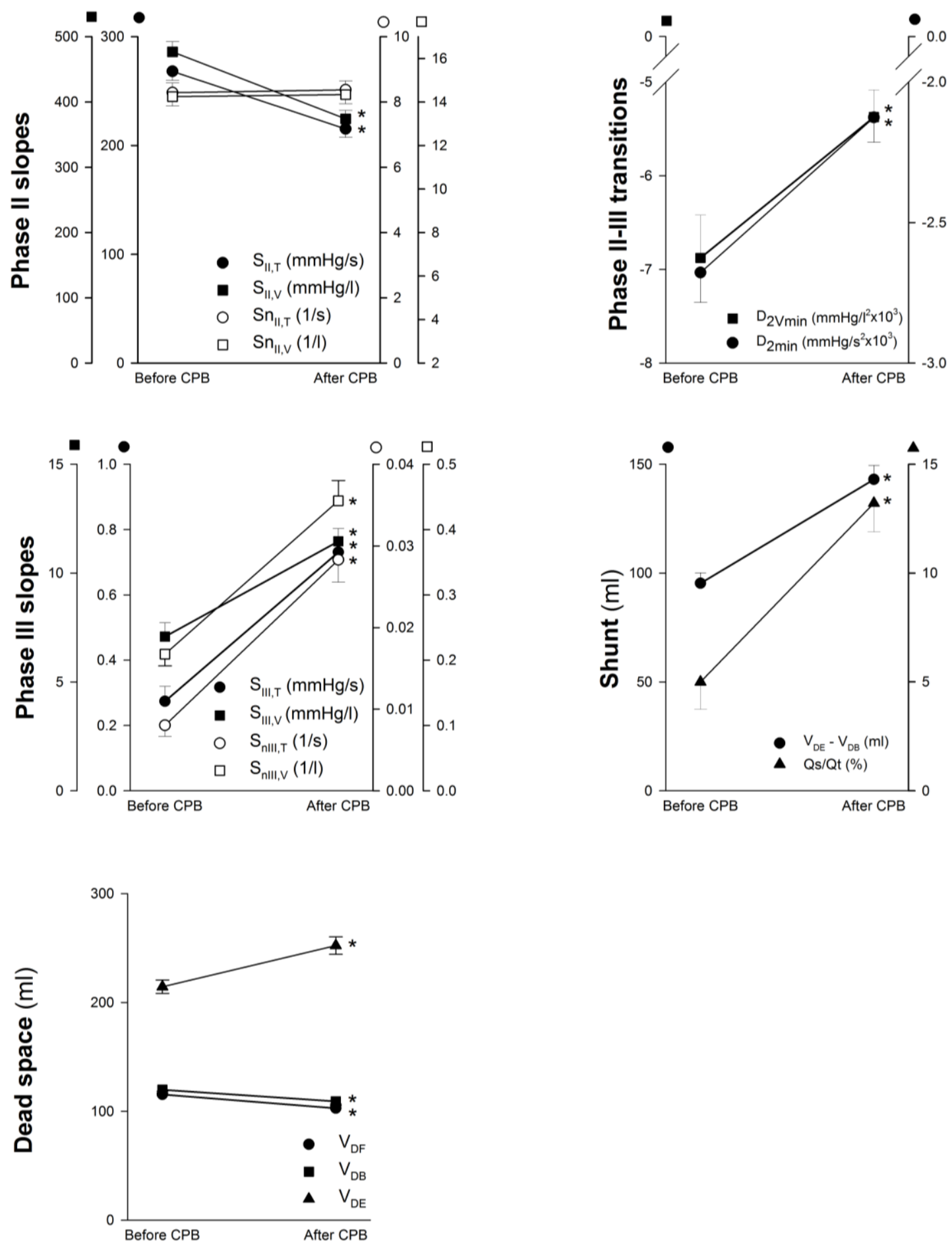

Figure 3. Indices derived from the time and volumetric capnographic measurements before and after the cardiopulmonary bypass (CPB). $S_{\mathrm{II}, \mathrm{T}, \mathrm{V}}$ and $\mathrm{Sn}_{\mathrm{II}, \mathrm{T}, \mathrm{V}}$ : absolute and normalized phase-II slopes of the time and volumetric capnogram, respectively. $S_{\text {III,T,V }}$ and $S_{\text {III,T,V: }}$ absolute and normalized phase-III slopes of the time and volumetric capnogram, respectively. $\mathrm{D}_{2 \min }$ and $\mathrm{D}_{2 \mathrm{Vmin}}$ : curvature at the phase II-III transitions, calculated as the minimum of the second-order time and volumetric derivative, respectively. $\mathrm{V}_{\mathrm{DF}}, \mathrm{V}_{\mathrm{DB}}$ and $\mathrm{V}_{\mathrm{DE}}$ denote dead spaces according to Fowler, Bohr and Enghoff. Qs/Qt: intrapulmonary shunt blood flow. Values are expressed as mean \pm SEM. 

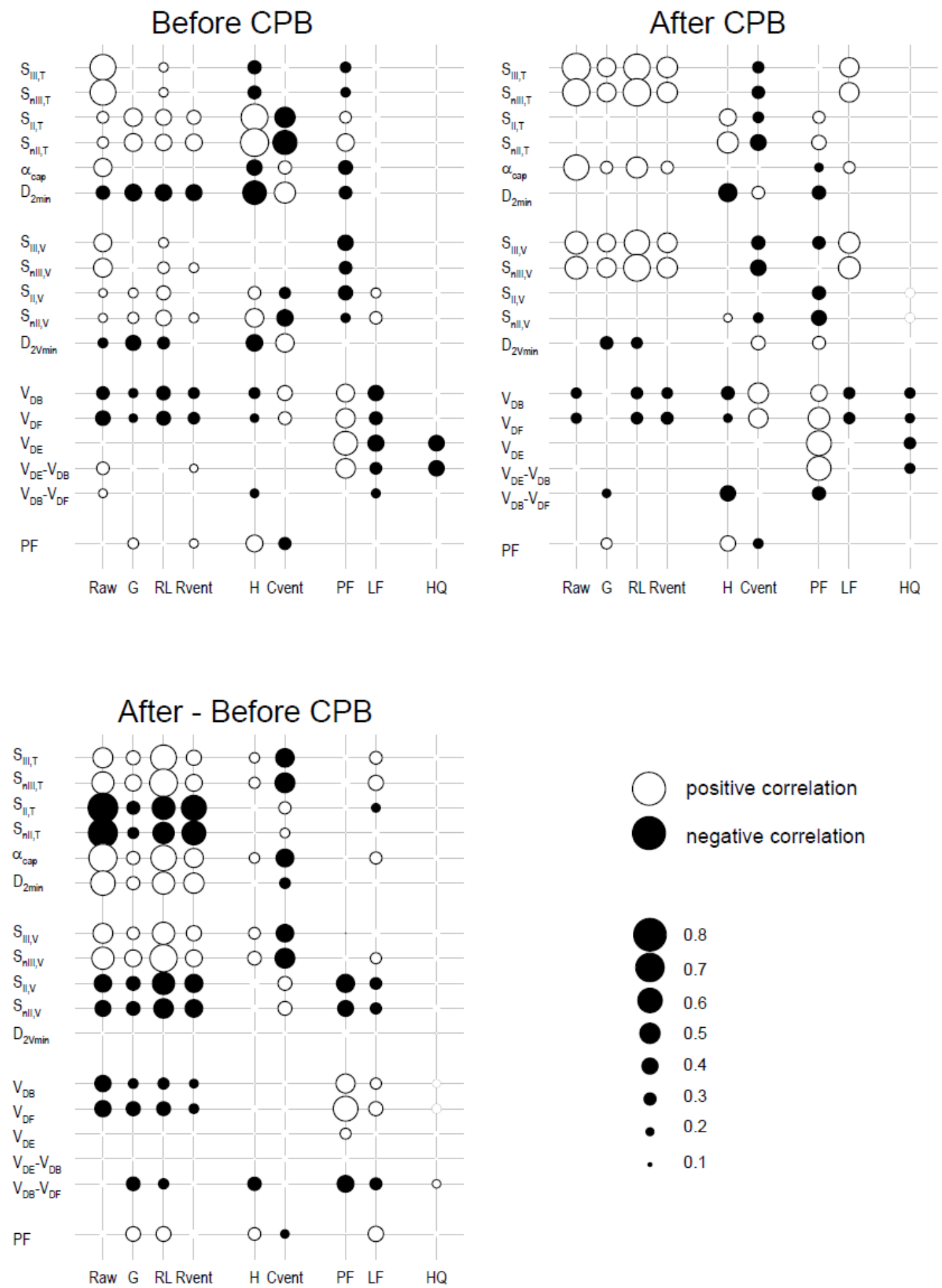

Figure 4. Strengths of correlations between the lung mechanical parameters (x-axis) and those obtained by time and volumetric capnography, reflecting the absolute and normalized slopes, and the absolute values reflecting transitions, dead space and shunt fractions (y-axis). The sizes of the circles denote the magnitude of the Pearson correlation coefficient. Open circles: significant positive correlation; closed circles: significant negative correlation; no circles: no significant correlation. 


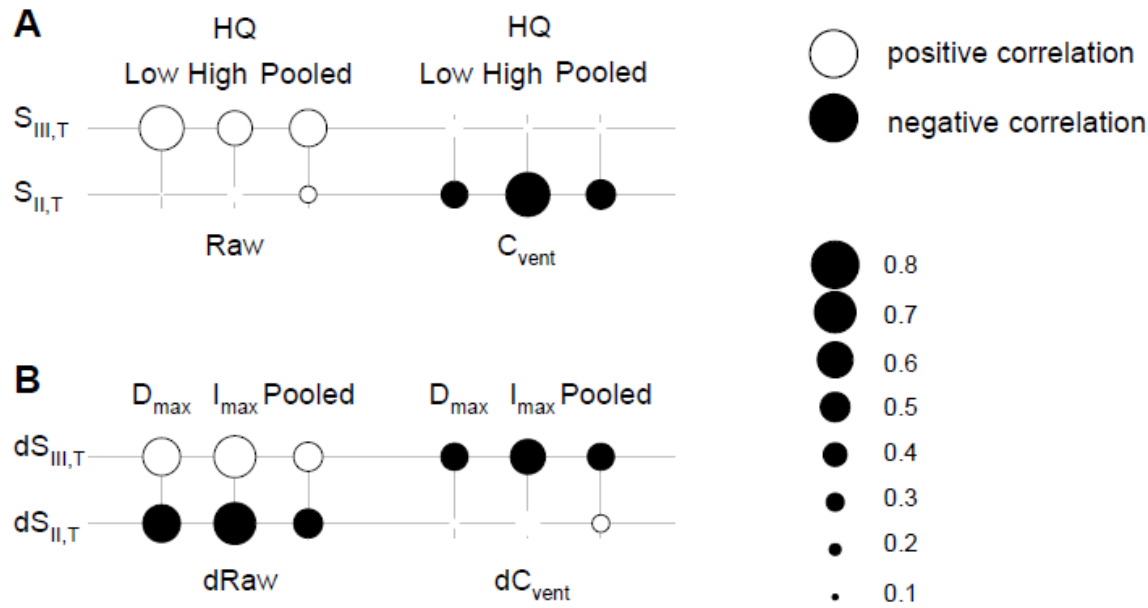

Figure 5. Strengths of correlations between the fundamental lung mechanical parameters (Raw and $\mathrm{C}_{\text {vent; }} \mathrm{x}$-axis) and phase II and III slopes obtained by time capnography (y-axis). Panel A: correlations between initial absolute values in the whole population (Pooled) and in subgroups with 25 percentile low and high initial HQ levels. Panel B: correlations between the changes in these parameters for the whole population (Pooled) and in subgroups with the highest 25 percentile increase $\left(I_{\max }\right)$, and lowest 25 percentile decrease $\left(\mathrm{D}_{\max }\right)$ in HQ after surgery. The sizes of the circles denote the magnitude of the Pearson correlation coefficient. Open circles: significant positive correlation; closed circles: significant negative correlation; no circles: no significant correlation. 


\section{ONLINE SUPPLEMENT \\ CAPNOGRAPHIC PARAMETERS IN VENTILATED PATIENTS: CORRESPONDENCE WITH AIRWAY AND LUNG TISSUE MECHANICS Zsofia Csorba ${ }^{1}$, Ferenc Petak ${ }^{2}$, Kitti Nevery ${ }^{1}$, Jozsef Tolnai ${ }^{2}$, Adam L. Balogh ${ }^{1,2}$, Ferenc Rarosi ${ }^{2}$, Gergely H Fodor ${ }^{2}$, Barna Babik ${ }^{1}$ \\ ${ }^{1}$ Department of Anesthesiology and Intensive Therapy \\ ${ }^{2}$ Department of Medical Physics and Informatics University of Szeged, Szeged, Hungary}

\section{METHODS}

\section{Patients' characteristics}

One hundred and one patients (age range 30-88 yrs) undergoing elective open heart surgery were examined in a prospective, consecutive manner. The heart surgery was necessitated by aortic $(n=70)$ and/or mitral $(n=27)$ valve disease combined with ischemic heart disease $(n=28)$, and/or other types of cardiac malformation $(n=6)$, myxoma or aneurysm of the ascending aorta. Based on earlier medical reports, the patients exhibited wide-ranging variations in pulmonary status: some had no pulmonary symptoms (i.e. no history of lung disease, a normal BMI, no pleural effusion, no pulmonary congestion, no smoking history, no wheezing periods within the past 6 months, and no history of the use of bronchodilator drugs; $n=16$ ), whereas others had lung abnormalities causing restrictive (pulmonary congestion $(n=56)$ and/or obesity $(B M I \geq 31) \quad(n=31))$ and/or obstructive changes (emphysema $(n=28)$, asthma $(n=8)$ or chronic bronchitis $(n=20))$.

\section{Cardiopulmonary bypass}

Prior to cardiopulmonary bypass (CPB), $1500 \mathrm{ml}$ of lactated Ringer's solution was used to prime the membrane oxygenator and the tube set. Heparin (300 IU/kg) was administered with the activated anticoagulation time maintained above $400 \mathrm{~s}$. At the beginning of the CPB, mild hypothermia was generally applied to maintain esophageal temperature of $32{ }^{\circ} \mathrm{C}$. During cardioplegic cardiac arrest, the lung ventilation was stopped, the ventilator was disconnected and no positive airway pressure was maintained in the lungs. The lungs were then inflated 3-5 times to a peak airway pressure of $30 \mathrm{cmH}_{2} \mathrm{O}$ before declamping of the aorta in order to facilitate the removal of gas emboli from the heart and to perform lung recruitment.

\section{Forced oscillatory measurements}

Airway and tissue mechanical properties were assessed by measuring the low-frequency forced oscillatory input impedance of the pulmonary system (ZL), as detailed previously (1). The common side of a T-piece was attached to a distal ET tube. The other sides of the Tpiece containing two collapsible segments were connected to the respirator and the forced oscillatory measurement apparatus. Before the oscillatory measurements, the lungs were inflated to a pressure of approximately $30 \mathrm{~cm} \mathrm{H}_{2} \mathrm{O}$ to standardize the volume history. During short (15-s) apneic periods, this equipment allowed switching the patient from the respirator to the forced oscillatory system while pseudorandom pressure excitations were generated into the trachea. The pressure forcing signal contained 15 integer-multiple components in the frequency range 0.4-6 Hz. ZL was computed from the power spectra of the airway opening pressure (Pao) and tracheal airflow (V'). Pao was measured with a pressure transducer (ICS model 33NA002D; ICSensors, Milpitas, CA, USA), and V' was measured with a 28-mm ID screen pneumotachograph connected to the identical pressure transducer. 
A well-validated 4-parameter model (2) containing a frequency-independent airway resistance (Raw) and inertance (Iaw) and a constant-phase tissue compartment characterized by the coefficients of damping $(\mathrm{G})$ and elastance $(\mathrm{H})$ was fitted to the mean $\mathrm{ZL}$ data by minimizing the weighted differences between the measured and modeled impedance values:

$\mathrm{ZL}=\mathrm{Raw}+\mathrm{j} \omega \mathrm{Iaw}+(\mathrm{G}-\mathrm{jH}) / \omega^{\alpha}$

where $\omega$ is the angular frequency $(2 \pi \mathrm{f})$ and $\alpha=2 / \pi \cdot \arctan (\mathrm{H} / \mathrm{G})$. The tissue resistive component (Rti) at the ventilation frequency $(0.2 \mathrm{~Hz})$ was calculated from the parenchymal damping coefficient $\left(\mathrm{Rti}=\mathrm{G} / \omega^{\alpha}\right)$. The total lung resistance $(\mathrm{RL})$ was determined as the sum of the airway resistance (Raw) and the Rti $\left(\mathrm{RL}=\mathrm{Raw}+\mathrm{G} / \omega^{\alpha}\right)$.

\section{Dead space measurements}

Fowler dead space

Fowler dead space $\left(\mathrm{V}_{\mathrm{DF}}\right)$, represents the anatomic dead-space volume of the conducting airways (Fig. 1). This was assessed by determining the inflection point on phase II of volumetric capnogram that separates the conductive and the alveolar space volumes $(3,4)$.

\section{Bohr dead space}

The physiological dead space, including also the alveolar volume not involved in gas exchange, was assessed by using the Bohr method $\left(\mathrm{V}_{\mathrm{DB}}\right)(5)$ :

$\mathrm{V}_{\mathrm{DB}}=\left(\mathrm{P}_{\mathrm{ACO}_{2}}-\mathrm{P}_{\overline{\mathrm{ECO}}}\right) / \mathrm{P}_{\mathrm{ACO}_{2}}$

where $\mathrm{P}_{\mathrm{ACO}_{2}}$ is the mean alveolar $\mathrm{CO}_{2}$ concentration located at the midpoint of the phase III in the expired $\mathrm{CO}_{2}$ curve, and $\mathrm{P}_{\overline{\mathrm{ECO}}}{ }_{2}$ is the mixed partial pressure of $\mathrm{CO}_{2}$ during the entire expiration $(6,7)$. The latter is calculated as the ratio of the tidal elimination of $\mathrm{CO}_{2}\left(\mathrm{VCO}_{2}\right)$ obtained by integrating the flow and $\mathrm{CO}_{2}$ signals over the entire breath and the tidal volume $(6,7)$.

\section{Enghoff dead space}

The dead space according to Enghoff's modification $\left(\mathrm{V}_{\mathrm{DE}}\right)$ takes also into account the ventilated but not perfused alveoli (8), and can therefore be calculated as

$\mathrm{V}_{\mathrm{DE}}=\left(\mathrm{P}_{\mathrm{aCO}_{2}}-\mathrm{P}_{\overline{\mathrm{ECO}}}\right) / \mathrm{P}_{\mathrm{aCO}}$

where $\mathrm{P}_{\mathrm{aCO}}$ is the partial pressure of $\mathrm{CO}_{2}$ in the arterial blood.

\section{Statistical analyses}

Standard error of means (SEM) was used to express scatters in measured variables. The normality of the data was tested with the Kolgomorov-Smirnov test with the Lilliefors correction. In the event of normality, paired t-tests were used to examine the statistical significance induced by CPB in the parameters. Wilcoxon signed-rank tests were utilized to verify the significance of the changes in the mechanical, capnographic or gas-exchange parameters. The Pearson test was applied to analyze the correlations between the different variables under each measurement condition, and to test the strength of the associations between the CPB-induced changes in the various parameters. The statistical tests were performed with a SigmaPlot statistical software package (Version 12.5, Systat Software, Inc. Chicago, IL, USA). All reported $\mathrm{p}$ values were two-sided. 


\section{SUPPLEMENTAL RESULTS}

The patients exhibited substantial interindividual variability in the lung mechanical and capnographic parameters (Table 1S), as reflected in the high coefficient of variation values before, after and the differences between after and before values.

\begin{tabular}{l|ccc} 
& Before & After & After-Before CPB \\
\hline Raw & 116 & 98 & 109 \\
$\mathrm{G}$ & 69 & 127 & 227 \\
$\mathrm{H}$ & 41 & 44 & 262 \\
$\mathrm{~S}_{\mathrm{nII}, \mathrm{T}}$ & 36 & 33 & 1163 \\
$\mathrm{~S}_{\mathrm{nIII}, \mathrm{T}}$ & 169 & 96 & 113 \\
$\mathrm{~V}_{\mathrm{DF}}$ & 26 & 24 & -134 \\
$\mathrm{~V}_{\mathrm{DB}}$ & 25 & 24 & -138 \\
$\mathrm{~V}_{\mathrm{DE}}$ & 28 & 32 & 156 \\
$\mathrm{~V}_{\text {DE }}-\mathrm{V}_{\mathrm{DB}}$ & 50 & 45 & 114
\end{tabular}

Table 1S. Coefficients of variations for the lung mechanical (Raw, $G$ and $H$ ) and capnographic parameters $\left(S_{\mathrm{nII}, \mathrm{T}}, \mathrm{S}_{\mathrm{nIII}, \mathrm{T}}, \mathrm{V}_{\mathrm{DF}}, \mathrm{V}_{\mathrm{DB}}, \mathrm{V}_{\mathrm{DE}}, \mathrm{V}_{\mathrm{DE}}-\mathrm{V}_{\mathrm{DB}}\right)$.

The key parameters obtained by forced oscillations and capnography for the individual patients are demonstrated on Fig. 1S (continuous thin lines), and for the group means (symbols with thick lines). Based on their starting pulmonary function, the cohort was divided into 3 groups: patients with the highest $25 \%$ (red), the medium $50 \%$ (grey) and the lowest 25\% HQ (green). There was no evidence for a statistical significance between the groups in the lung mechanical parameters (Raw, $\mathrm{G}$ and $\mathrm{H}$ ) and capnographic shape factors

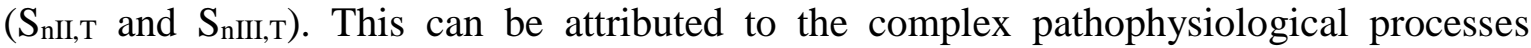
involved in the gas exchange, including ventilation, perfusion and ventilation/perfusion. Accordingly, there is no direct link between individual lung mechanical or capnogram parameters with gas exchange indices. Conversely, capnographic parameters primarily affected by lung perfusion $\left(\mathrm{V}_{\mathrm{DF}}, \mathrm{V}_{\mathrm{DB}}, \mathrm{V}_{\mathrm{DE}}\right.$ and $\left.\mathrm{V}_{\mathrm{DE}}-\mathrm{V}_{\mathrm{DB}}\right)$ exhibit statistically significant differences in the different HQ groups. 
Figure 1S
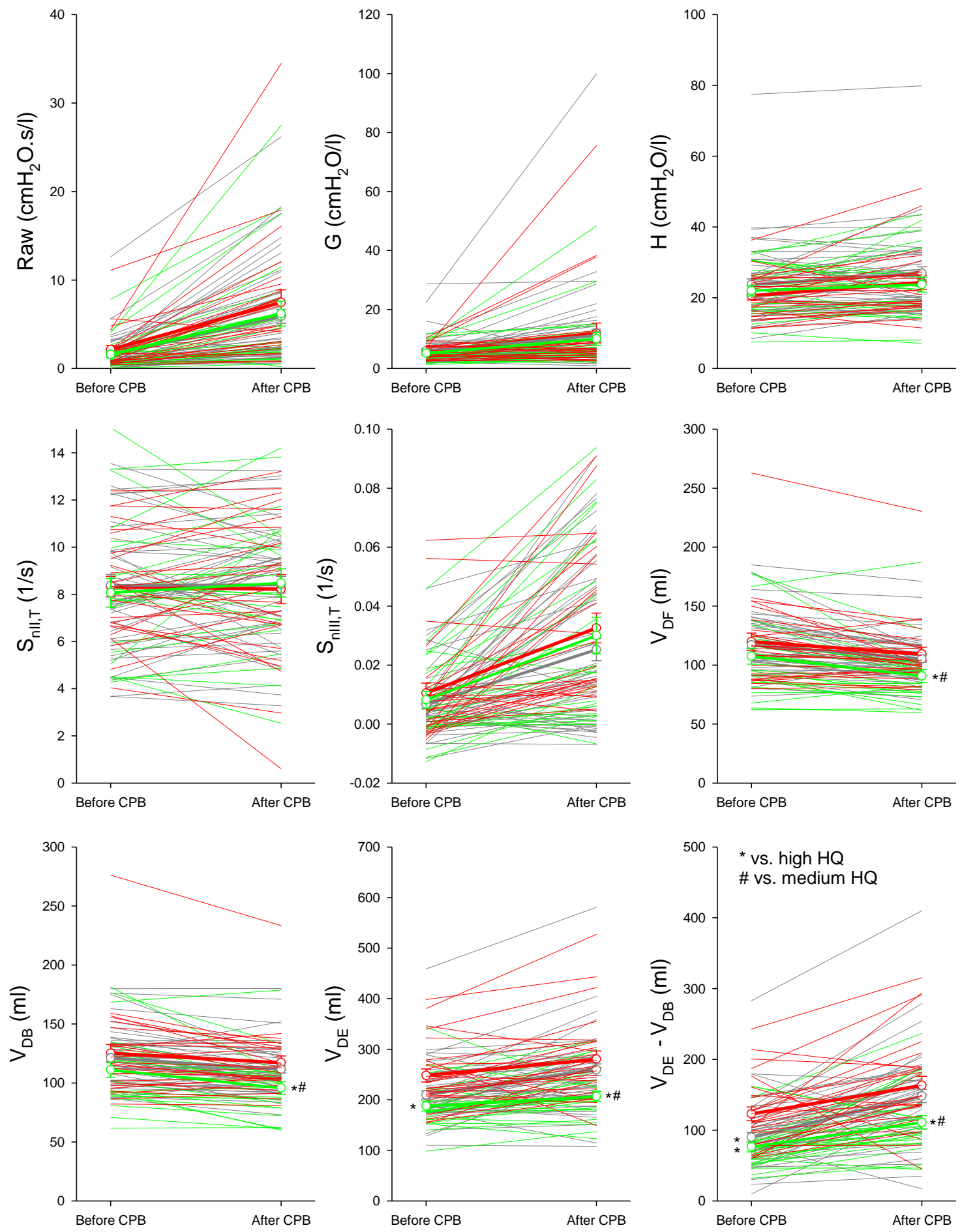

The interdependence of the main shape factors obtained from the time capnogram with lung mechanical parameters representing the airway resistance (Raw) and lung elastance $(\mathrm{H})$ under the baseline conditions are demonstrated in Fig. 2S. The magnitude of $S_{\text {nIII,T }}$ depends more on Raw than on $\mathrm{H}$ (Panel A), whereas the level of $S_{\mathrm{nII}, \mathrm{T}}$ appears to be determined primarily by $\mathrm{H}$, with lower correlations with Raw (Panel B). The capnographic parameters 
expressing the transition from phase II to phase III $\left(\mathrm{D}_{2 \mathrm{~min}}\right)$ displayed stronger, but opposite dependence on $\mathrm{H}$ than on Raw (Panel C).
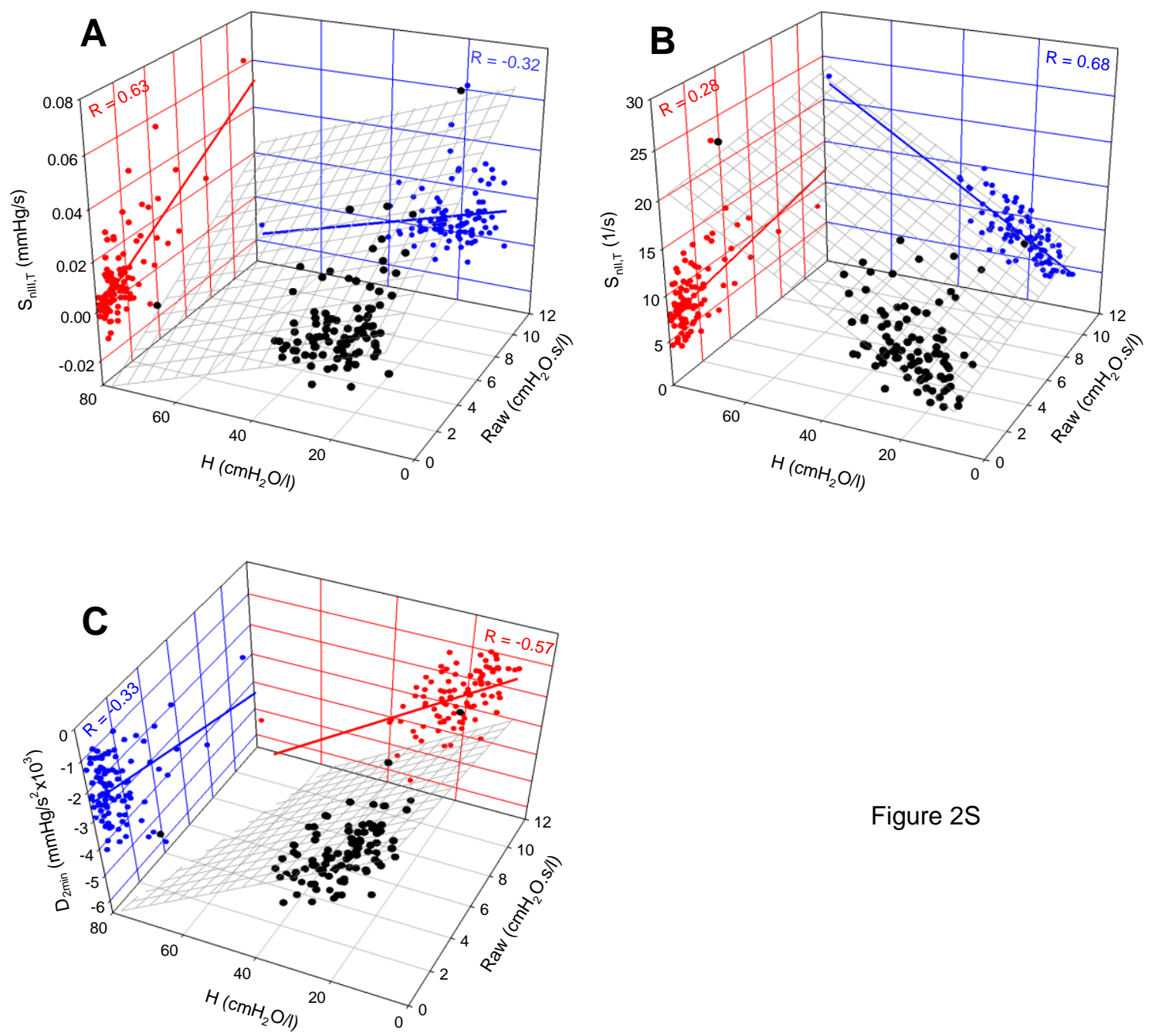

Figure 2S

\section{REFERENCES}

1. Babik B, Asztalos T, Petak F, Deak ZI, Hantos Z. Changes in respiratory mechanics during cardiac surgery. Anesthesia and analgesia 2003;96:1280-7, table of contents.

2. Hantos Z, Daroczy B, Suki B, Nagy S, Fredberg JJ. Input impedance and peripheral inhomogeneity of dog lungs. Journal of applied physiology 1992;72:168-78.

3. Fowler WS. Lung function studies; the respiratory dead space. The American journal of physiology 1948;154:405-16.

4. Fowler WS. Respiratory dead space. Federation proceedings 1948;7:35.

5. $\quad$ Bohr C. Über die Lungenatmung. Skan Arch Physiol 1891;53:236-8.

6. Tusman G, Sipmann FS, Bohm SH. Rationale of dead space measurement by volumetric capnography. Anesthesia and analgesia 2012;114:866-74.

7. Tusman G, Scandurra A, Bohm SH, Suarez-Sipmann F, Clara F. Model fitting of volumetric capnograms improves calculations of airway dead space and slope of phase III. Journal of clinical monitoring and computing 2009;23:197-206.

8. Enghoff H. Volumen inefficax. Uppsala Laekareforen Forh 1938;44:191-218. 
II. Babik B, Csorba Z, Czövek D, Mayr PN, Bogáts G, Peták F. Effects of respiratory mechanics on the capnogram phases: importance of dynamic compliance of the respiratory system. Crit Care. 16(5):R177, 2012 [IF: 4.718] 


\title{
Effects of respiratory mechanics on the capnogram phases: importance of dynamic compliance of the respiratory system
}

Barna Babik', Zsófia Csorba ${ }^{1}$, Dorottya Czövek², Patrick N Mayr ${ }^{3}$, Gábor Bogáts ${ }^{4}$ and Ferenc Peták²

\begin{abstract}
Introduction: The slope of phase III of the capnogram $\left(S_{\| I}\right)$ relates to progressive emptying of the alveoli, a

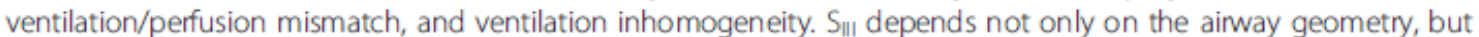
also on the dynamic respiratory compliance (Crs); this latter effect has not been evaluated. Accordingly, we established the value of $\mathrm{S}_{\| 1}$ for monitoring airway resistance during mechanical ventilation.

Methods: Sidestream capnography was performed during mechanical ventilation in patients undergoing elective cardiac surgery $(n=144)$. The airway resistance (Raw), total respiratory resistance and Crs displayed by the ventilator, the partial pressure of arterial oxygen $\left(\mathrm{PaO}_{2}\right)$ and $\mathrm{S}_{\| I}$ were measured in time domain $\left(\mathrm{S}_{\mathrm{T}-111}\right)$ and in a smaller cohort $(n=68)$ by volumetry $\left(\mathrm{S}_{\mathrm{V}-\mathrm{II}}\right)$ with and without normalization to the average $\mathrm{CO}_{2}$ phase III concentration. Measurements were performed at positive end-expiratory pressure (PEEP) levels of 3,6 and 9 $\mathrm{CmH}_{2} \mathrm{O}$ in patients with healthy lungs (Group $\mathrm{HL}$ ), and in patients with respiratory symptoms involving low (Group LC), medium (Group MC) or high Crs (Group HC).

Results: $\mathrm{S}_{\mathrm{T}-\| I}$ and $\mathrm{S}_{\mathrm{V}-\mathrm{II}}$ exhibited similar PEEP dependencies and distribution between the protocol groups formed on the basis of $\mathrm{Cr}$. A wide interindividual scatter was observed in the overall Raw-ST-II relationship, which was primarily affected by Crs. Decreases in Raw with increasing PEEP were reflected in sharp falls in $S_{\text {III }}$ in Group HC, and in moderate decreases in $S_{\| I}$ in Group MC, whereas $S_{T-I I I}$ was insensitive to changes in airway caliber in Groups $\mathrm{LC}$ and $\mathrm{HL}$

Conclusions: $\mathrm{S}_{\| \mid}$assessed in the time domain and by volumetry provide meaningful information about alterations in airway caliber, but only within an individual patient. Although $\mathrm{S}_{\text {T-III }}$ may be of value for bedside monitoring of the airway properties, its sensitivity depends on Crs. Thus, assessment of the capnogram shape should always be coupled with Crs when the airway resistance or oxygenation are evaluated.
\end{abstract}

\section{Introduction}

Capnography is a noninvasive, continuous, online, dynamic, effort- and cooperation-independent method for bedside monitoring of the exhaled carbon dioxide $\left(\mathrm{CO}_{2}\right)$ concentration. The slope of the third phase of the capnogram $\left(\mathrm{S}_{\mathrm{III}}\right)$ is determined physiologically by the continuous $\mathrm{CO}_{2}$ excretion from the pulmonary vasculature and the periodic lung ventilation $[1,2]$, and the interactions between the diffusive and convective gas mixing [3-5]. The development of pathophysiological

* Correspondence: petakferenc@med.u-szeged.hu

${ }^{2}$ Department of Medical Physics and Informatics, University of Szeged, 9

Korányi fasor, H-6720 Szeged, Hungary

Full list of author information is available at the end of the article ventilatory and/or perfusion inhomogeneities in time and space leads to lung compartments containing various $\mathrm{CO}_{2}$ concentrations, and this may further elevate $\mathrm{S}_{\text {III }}[2,6-13]$.

The sensitivity of $\mathrm{S}_{\text {III }}$ to ventilation/perfusion abnormalities suggested its clinical usefulness for the detection of respiratory abnormalities or the following of ventilatory and/or pharmacological interventions. Numerous studies have demonstrated that the magnitude of $\mathrm{S}_{\text {III }}$ reflects the severity of emphysema or asthma [4,11,13-17], cystic fibrosis and bronchiectasis [8], chronic obstructive pulmonary disease (COPD) [7], chronic bronchitis [9] and acute lung injury $[6,10]$. Inconsistent associations have been reported in previous attempts to clarify the quantitative relationships

O 2012 Babik et al; licensee BioMed Central Ltd This is an open access article distributed under the terms of the Greative Commons 
between capnographic and lung function indices. Earlier studies reported a strong correlation between the forced expiratory volume in one second $\left(\mathrm{FEV}_{1}\right)$ and $\mathrm{S}_{\mathrm{III}}[15]$, merely a modest association $[11,16]$, or even a lack of correspondence [13]. Furthermore, significant correlations were observed between the total respiratory resistance (Rrs) and $\mathrm{S}_{\mathrm{III}}$ in mechanically ventilated patients, however $\mathrm{S}_{\text {III }}$ had limited clinical applicability to predict Rrs [18]. Thus, in consequence of the complex mechanisms affecting $\mathrm{S}_{\mathrm{II}}$, its diagnostic and/or monitoring value is far from being clear. The diverse emptying of different lung compartments with various $\mathrm{CO}_{2}$ levels is determined not only by the airway geometry, but also by the driving pressure governed by the dynamic respiratory compliance (Crs), including the chest wall and the lung. Despite the obvious importance of respiratory tissue elastance in determining the expiratory flow and the rate of $\mathrm{CO}_{2}$ clearance, the role of the respiratory elastic recoil on the capnogram shape has not been examined to date.

The aim of the present prospective consecutive clinical study was to investigate systematically whether the capnogram shape is affected by changes in both airway caliber and the Crs in mechanically ventilated patients. We also set out to clarify the contribution of the altered airway properties and tissue mechanics with increasing positive end-expiratory pressure (PEEP) to the changes in $\mathrm{S}_{\text {III. }}$ To test the hypothesis that both the airway geometry and the Crs reflecting the elastic recoil of the respiratory system affect $\mathrm{S}_{\mathrm{III}}$, a large cohort of mechanically ventilated patients with normal and diseased lungs was studied.

\section{Materials and methods \\ Patients}

One hundred and forty-four patients (93 males, 51 female, $62 \pm 9($ mean $\pm S D)$ years of age (range 39 to 84 years)) undergoing elective coronary bypass surgery were examined in the supine position before the surgical procedure. The protocol was approved by the Human Research Ethics Committee of Szeged University, Hungary (no. WHO 2788), and the patients gave their informed consent to the study. The patients were premedicated with intramuscular morphine $(0.07 \mathrm{mg} / \mathrm{kg})$ and midazolam $(0.07 \mathrm{mg} / \mathrm{kg}) 1 \mathrm{~h}$ before the operation. Anesthesia was induced with i.v. midazolam $(30 \mu \mathrm{g} / \mathrm{kg})$, sufentanil $(0.4$ to $0.5 \mu \mathrm{g} / \mathrm{kg})$ and propofol $(0.3$ to $0.5 \mathrm{mg} / \mathrm{kg})$. Muscle paralysis was achieved with an i.v. bolus of rocuronium $(0.6 \mathrm{mg} / \mathrm{kg})$. The anesthesia and muscle relaxation were maintained with i.v. infusions of propofol $(50 \mu \mathrm{g} / \mathrm{kg} / \mathrm{min})$ and i.v. boluses of rocuronium $(0.2 \mathrm{mg} / \mathrm{kg}$ every $30 \mathrm{~min})$.

The trachea was intubated with a cuffed endotracheal tube with an internal diameter of 7,8 or $9 \mathrm{~mm}$, and the patients were ventilated with a Dräger Zeus anesthesia machine (Lübeck, Germany) in volume-controlled mode with descending flow. The ventilator frequency was set to 12 to 14 breaths $/ \mathrm{min}$, a tidal volume of $7 \mathrm{ml} / \mathrm{kg}$ and a PEEP of $3 \mathrm{cmH}_{2} \mathrm{O}$ were applied. The fraction of inspired oxygen $\left(\mathrm{FiO}_{2}\right)$ was maintained at 0.5 throughout the entire study period. Arterial blood gas samples were analyzed at least hourly (Radiometer ABLTM 505, Copenhagen, Denmark). The ejection fraction (EF) data were collected from preoperative echocardiography. The body mass index (BMI) of each patient was calculated.

The patients had various cardiac diseases including ischemic heart disease $(n=108)$, a mitral insufficiency $(n=21)$, aortic stenosis $(n=38)$, and other types of cardiac malformation $(n=5)$, such as myxoma, congenital heart disease or aortic aneurysm. The patients exhibited wide variations in their pulmonary status, with some of them having no pulmonary symptoms (that is, no history of lung disease, normal BMI, no pleural effusion, no pulmonary congestion, no smoking history, lack of wheezing periods within the past six months, lack of use of bronchodilator drugs; $n=29$ ). Others had lung abnormalities causing restrictive (pulmonary congestion $(n=45)$ or obesity (BMI $\geq 31 ; n=48)$ ) and/or obstructive changes (emphysema $(n=55)$, asthma $(n=14)$, chronic bronchitis $(n=25)$ or sarcoidosis $(n=1))$.

To establish whether the elastic properties of the respiratory system affected the capnogram shape, the patients with respiratory symptoms were allocated into three groups, on the basis of their Crs. This Crs was determined $10 \mathrm{~min}$ after anesthesia induction and a lung volume homogenization maneuver (that is, lung inflation and maintenance at a transrespiratory pressure of 30 $\mathrm{cmH}_{2} \mathrm{O}$ for $5 \mathrm{~s}$ ) when stable hemodynamic and ventilatory conditions at PEEP $3 \mathrm{cmH}_{2} \mathrm{O}$ have been reached (that is, prior to the first capnogram recording). Group LC comprised patients with Crs in the lowest tenth percentile ( $\left.\mathrm{Crs}<34.5 \mathrm{ml} / \mathrm{cmH}_{2} \mathrm{O} ; n=15\right)$, and Group MC patients with $\mathrm{Crs}$ between the tenth and the ninetieth percentile ( $\left.34.5<\mathrm{Crs}<69 \mathrm{ml} / \mathrm{cmH}_{2} \mathrm{O} ; n=85\right)$, and Group $\mathrm{HC}$ patients with $\mathrm{Crs}$ above the ninetieth percentile $\left(\mathrm{Crs}>69 \mathrm{ml} / \mathrm{cmH}_{2} \mathrm{O} ; n=15\right)$. Patients with healthy lungs were regarded as an independent group (Group HL; $n=29$ ). The patients were classified based on the Crs measured after a lung volume recruitment maneuver. The characteristics of the patients in each protocol group are summarized in Table 1. The age of the patients did not differ significantly in the different groups $(P=0.16)$.

\section{Measurement of airway and respiratory tissue mechanics}

Details of the measurement of the input impedance of the respiratory system (Zrs) were reported previously [19]. Briefly, a T-piece with two collapsible segments was attached to the distal endotracheal tube, with one end connected to the respirator and the other end to a loudspeaker-in-box system. This apparatus allowed switching 
Babik et al. Gritical Care 2012, 16:R177

Page 3 of 10

http://ccforum.com/content/16/5/R177

Table 1 Number of patients with different conditions/diagnoses in each protocol group.

\begin{tabular}{lcccc}
\hline & Gender* $^{*}(\mathbf{m} / \mathbf{f})$ & Obesity* $(\mathbf{n} /$ ow/ob) & Pulmonary status E*/A*/CB*/OD & Cardiac disease CAD/AS/MI/LEF*/OCD \\
\hline Group HL $(\boldsymbol{n}=\mathbf{2 9})$ & $20 / 9$ & $10 / 19 /-$ & $-/ /-/-$ & $26 / 3 / 3 /-/ 1$ \\
\hline Group HC $(\boldsymbol{n}=\mathbf{1 5})$ & $15 /-$ & $8 / 7 /-$ & $14 / / / 6 /-$ & $11 / 4 /-/--$ \\
\hline Group MC $(\boldsymbol{n}=\mathbf{8 4})$ & $56 / 28$ & $12 / 28 / 44$ & $37 / 10 / 15 / 2$ & $61 / 28 / 8 / 10 / 3$ \\
\hline Group LC $(\boldsymbol{n}=\mathbf{1 5})$ & $3 / 12$ & $-/ 5 / 10$ & $4 / 4 / 4 /-$ & $10 / 3 / 1 / 8 / 1$ \\
\hline
\end{tabular}

Obesity categories: $\mathrm{n}$, normal $(20 \leq \mathrm{BMI}<25)$; ow, overweight $(25 \leq \mathrm{BMI}<30)$; ob, obese $(30 \leq \mathrm{BMI})$. Pulmonary status: $\mathrm{E}$, emphysema; $\mathrm{A}$, asthma; $\mathrm{CB}$, chronic bronchitis; OLD, other lung disease. Cardiac disease: CAD, coronary artery disease; $A S$, aortic stenosis; ML, mitral insufficiency; LEF, low ejection fraction (EF < 50\%); OCD, other cardiac disease. Pulmonary and cardiac conditions are based on previous clinical diagnoses. $*, P<0.05$ between the expected and the observed frequencies in the protocol groups for each variable. Group HC, group of patients with high dynamic respiratory compliance; Group HL, group of patients with healthy lungs; Group LC, group of patients with low dynamic respiratory compliance; Group MC, group of patients with medium dynamic respiratory compliance.

of the patient from the respirator to the forced oscillatory setup during the recordings. These were performed by generating pseudorandom pressure excitations into the trachea during short ( $15 \mathrm{~s}$ ) apneic pauses superimposed into the mechanical ventilation. The forcing signal contained 30 integer-multiple components of the $0.2 \mathrm{~Hz}$ fundamental frequency, in the frequency range 0.2 to $6 \mathrm{~Hz}$. Tracheal airflow $\left(\mathrm{V}^{\prime}\right)$ was measured with a $28 \mathrm{~mm}$ ID screen pneumotachograph connected to a differential pressure transducer (ICS model 33NA002D; ICSensors, Milpitas, CA, USA). The airway opening pressure ( $\mathrm{Pao}$ ) was detected with an identical pressure transducer. Zrs was computed from the power spectra of Pao and V', and then ensemble-averaged under each condition. The mean Zrs data were fitted by a well-validated four-parameter model [20] containing a frequency-independent airway resistance (Raw) and inertance (Iaw) and a constantphase tissue compartment characterized by the coefficients of damping $(\mathrm{G})$ and elastance $(\mathrm{H})$.

\section{Recording and analyses of the capnogram}

Changes in partial $\mathrm{CO}_{2}$ pressure in the exhaled gas during mechanical ventilation were measured with a calibrated sidestream capnometer (Ultima ${ }^{\mathrm{TM}}$, Datex/Instrumentarium, Helsinki, Finland). Since capnograms are displayed in clinical routine in the time domain, time capnography was applied in each patient to record $\mathrm{CO}_{2}$ changes. To minimize the possible drawback of this time domain analyses, we paid attention to involve only the linear part of the $\mathrm{CO}_{2}$ trace in the readings of $\mathrm{S}_{\mathrm{III}}$. Nevertheless, volumetric capnography may allow a better distinction between the phases $[2,4,6,7,10,12,14,21]$ and thus, in a subgroup including the last 68 patients, the flow during mechanical ventilation was simultaneously recorded with the $\mathrm{CO}_{2}$ traces by introducing an additional pneumotachograph into the ventilation circuit. This allowed the analyses of volumetric capnograms in 20, 7, 32 and 9 patients in the Groups HL, $\mathrm{HC}, \mathrm{MC}$ and $\mathrm{LC}$, respectively. The $15 \mathrm{~s} \mathrm{CO}_{2}$ and respiratory flow traces were imported into commercial signal analysis software (Biopac, Santa Barbara, CA, USA). Linear regression analysis was applied to obtain the slope of the third phase of the expiratory capnogram in the time domain $\left(\mathrm{S}_{\mathrm{T}-\mathrm{III}}\right)$ and $\mathrm{CO}_{2}$ concentration was analyzed as a function of expired volume to obtain the volumetric third phase slope $\left(\mathrm{S}_{\mathrm{v}-\mathrm{III}}\right)$. These analyses were performed by fitting a line for each expiratory phase in the recordings in the linear phase before the end-expiratory peak $[6,11]$. Both $\mathrm{S}_{\mathrm{T} \text {-III }}$ and $\mathrm{S}_{\mathrm{V} \text {-III }}$ were normalized by dividing each slope by the average values of the corresponding $\mathrm{CO}_{2}$ concentration in mixed expired gas to obtain normalized time domain $\left(\mathrm{Sn}_{\mathrm{T} \text {-III }}\right)$ and volumetric $\left(\mathrm{Sn}_{\mathrm{V} \text {-III }}\right)$ third phase slopes $[2,21,22]$. Three to four expiratory traces were analyzed in each recording resulting in an ensemble averaging of 10 to 12 values under each condition.

\section{Measurement protocol}

The scheme of the experimental protocol is outlined in Figure 1. When stable hemodynamic and respiratory mechanical conditions had been reached while PEEP was maintained at $3 \mathrm{cmH}_{2} \mathrm{O}$, an arterial blood gas sample was taken, and dynamic compliance (Crs) was recorded from the display of the respirator. The first capnogram trace was then collected followed by recording of the first Zrs data epoch. Two more capnographic and Zrs measurements were then made in alternating sequence at $60 \mathrm{~s}$ intervals. PEEP was next elevated to 6 and then $9 \mathrm{cmH}_{2} \mathrm{O}$, a $3 \mathrm{~min}$ equilibration period being permitted after each step, and the data collection procedure was repeated.

\section{Statistical analyses}

Scatters in measured variables are expressed as SE. The normality of the data was tested with the KolgomorovSmirnov test with Lilliefors correction. Two-way repeated measures analysis of variance (ANOVA) including an interaction term was used with the variables PEEP $(3,6$ and $9 \mathrm{cmH}_{2} \mathrm{O}$ ) and the group allocation (Groups $\mathrm{HL}, \mathrm{LC}$, $\mathrm{MC}$ and $\mathrm{HC}$ ) to establish the effects of lung volume and $\mathrm{Crs}$ on the respiratory mechanical, blood gas and capnographic variables. This statistical method was utilized to test the hypothesis that the level of Crs affects the PEEPdependent changes in the respiratory mechanical and 


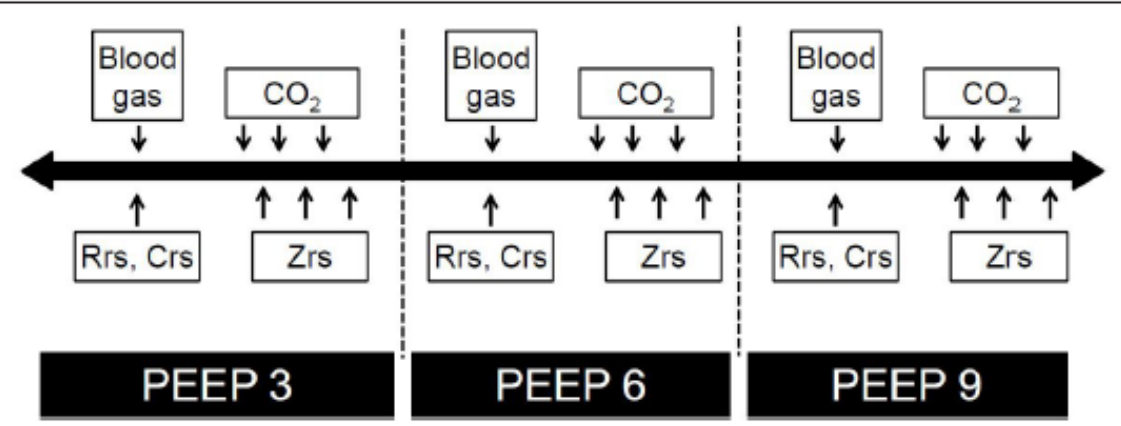

Figure 1 Timeline of the experimental protocol. Rrs and $\mathrm{Crs}$, readings from the respirator display; $\mathrm{CO}_{2}$, recording of capnogram curves; $\mathrm{Zrs}$, forced oscillatory measurement of respiratory system impedance. $\mathrm{CO}_{2}$, carbon dioxide; $\mathrm{Grs}$, dynamic respiratory compliance; Rrs, total respiratory resistance; $Z \sqrt{5}$, input impedance of the respiratory system.

capnogram variables. Multiple linear regression analysis was performed to establish whether the levels of BMI and $\mathrm{EF}$ affect Crs. The Holm-Sidak multiple comparison procedure was adopted to compare the variables in the various study groups under different conditions. Chisquare test was used to assess whether there is a significant difference between the expected and the observed frequencies of gender, obesity, pulmonary and cardiac diseases in the protocol groups. The correlation between $\mathrm{S}_{\mathrm{T} \text {-III }}$ and $\mathrm{S}_{\mathrm{V} \text {-III }}$ were analyzed by Pearson test. The statistical tests were performed with a SigmaPlot statistical software package (Version 12, Systat Software, Inc. Chicago, IL, USA). All reported $P$ values are two-sided.

\section{Results}

The changes in the respiratory mechanical parameters, the partial pressure of arterial oxygen $\left(\mathrm{PaO}_{2}\right)$ and the indices obtained from the capnograms with increasing PEEP in the four groups of patients are depicted in Figure 2. The statistical analyses revealed significant interactions between the group allocation and PEEP, demonstrating that the respiratory compliance exerted significant effects on the responses to PEEP in the forced oscillatory mechanical parameters $(P<0.001$ for Raw, G and $\mathrm{H})$, for the $\mathrm{Crs}$ displayed by the respirator $(P<0.001), \mathrm{PaO}_{2}(P=$ $0.04)$, and the capnogram third phase slope variables $(P<$ 0.001 for $\mathrm{S}_{\mathrm{T}-\mathrm{III}}$ and $\mathrm{Sn}_{\mathrm{T}-\mathrm{III}}, P=0.003$ for $\mathrm{S}_{\mathrm{V}-\mathrm{III}}$, and $P=$ 0.002 and $\left.\mathrm{Sn}_{\mathrm{V}-\mathrm{III}}\right)$. Time and volumetric capnogram variables exhibited similar Crs and PEEP dependences, which is also reflected in the significant correlations between $\mathrm{S}_{\mathrm{T}-\mathrm{III}}$ and $\mathrm{S}_{\mathrm{V}-\mathrm{III}}$ in Groups HL $(\mathrm{R}=0.4, P=0.002), \mathrm{HC}$ $(\mathrm{R}=0.79, P<0.001), \mathrm{LC}(\mathrm{R}=0.45, P=0.02)$ and $\mathrm{MC}(\mathrm{R}=$ $0.79, P<0.001)$.

The greatest Raw, G, $\mathrm{H}$ and the lowest $\mathrm{PaO}_{2}$ were observed for the patients in Group LC, and these patients generally exhibited the greatest response to PEEP. The patients in Group MC still exhibited elevated Raw, G and $\mathrm{H}$ with a more moderate, but still significant response to
PEEP changes. The lowest forced oscillatory airway and tissue parameters and the greatest $\mathrm{PaO}_{2}$ were obtained in the patients in Groups HL and $\mathrm{HC}$, and their changes with PEEP were generally mild. The capnogram third phase indices were highest in Group $\mathrm{HC}$ and somewhat lower in Group MC, with both groups exhibiting marked decreases with increasing PEEP. The variables characterizing the third phase slopes from the capnogram were lowest in the patients in Group HL.

Figure 3 depicts the relationship of Raw and $\mathrm{S}_{\mathrm{T}-\mathrm{III}}$ in the individual patients and the group means for the four protocol groups following the increases of PEEP. In all patients, Raw and $\mathrm{S}_{\mathrm{T} \text {-III }}$ underwent concomitant monotonous decreases with increasing PEEP, but marked differences were observed between the protocol groups in the relationships of these parameters. The marked decreases in the high initial Raw values were associated with substantially smaller drops in $\mathrm{S}_{\mathrm{III}}$ in the patients in Group LC, whereas the PEEP-induced decreases in $\mathrm{S}_{\mathrm{III}}$ were more pronounced than those in Raw in the patients in Group HC. The patients in Group MC exhibited an intermediate Raw-S $\mathrm{S}_{\mathrm{T}-\mathrm{III}}$ relationship. This trend of association was observed in the patients in Group HL at markedly lower levels of Raw and $\mathrm{S}_{\mathrm{III}}$.

To examine the possible roles of obesity and lung congestion in the increased level of Crs, the effects of BMI and EF were considered (Figure 4). The patients in Group LC had significantly higher BMI $(P<0.001)$ and/or lower EF $(P<0.001)$ than those in Groups HL or $\mathrm{HC}$, indicating that the low $\mathrm{Crs}$ was a consequence of restrictive changes resulting from obesity and/or heart failure leading to pulmonary congestion (multiple linear regression coefficient of $\mathrm{R}=0.58$ ). The important effects of BMI and EF on the group allocation was confirmed by the presence of a significant correlation ( $\mathrm{R}=0.53, P=0.005$ and $P<0.0001$ for $\mathrm{EF}$ and $\mathrm{BMI}$, respectively). 

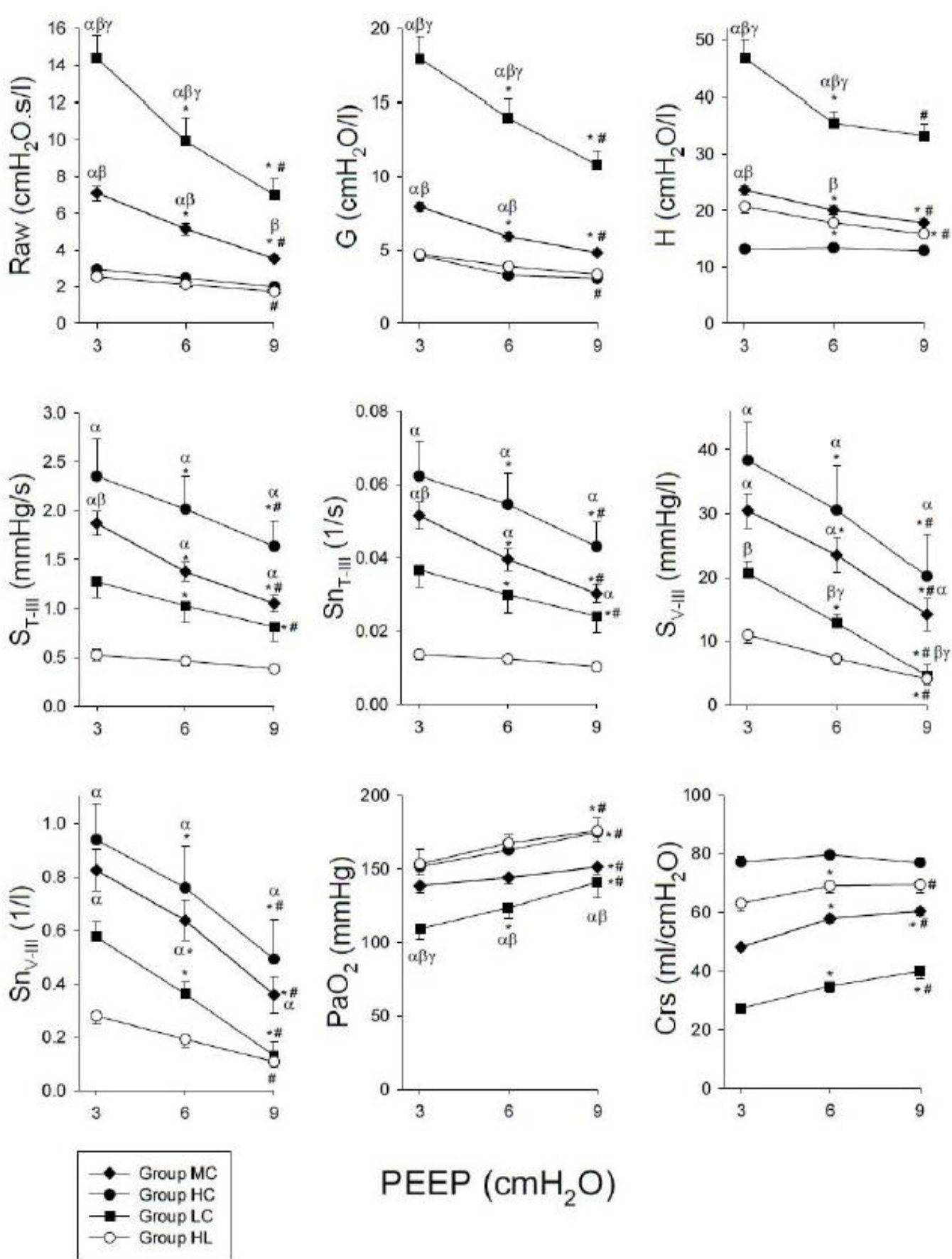

PEEP $\left(\mathrm{cmH}_{2} \mathrm{O}\right)$

Figure 2 Forced oscillatory airway (Raw, airway resistance) and respiratory tissue (G, damping and $\mathrm{H}$, elastance) mechanica parameters, the slope of the third phase of the capnogram as expressed in the time domain before $\left(\mathrm{S}_{\mathrm{T}-\mathrm{II}}\right)$ and after normalization for the mean expired $\mathrm{CO}_{2}\left(\mathrm{Sn}_{\mathrm{TAII}}\right)$, or as a function of expired volume before $\left(\mathrm{S}_{\mathrm{VAII}}\right)$ and after $\left(\mathrm{Sn}_{\mathrm{y}-\mathrm{II}}\right)$ normalization, partial pressure of oxygen in the arterial blood $\left(\mathrm{PaO}_{2}\right)$ and dynamic compliance $(\mathrm{Crs})$ displayed by the respirator in patients with healthy lungs (Group $\mathrm{HL}$ ), and in patients with respiratory symptoms with Crs in the lowest tenth percentile (Group LC), with Crs between the tenth and the ninetieth percentile (Group MC) and with $\mathrm{Crs}$ above the ninetieth percentile in (Group $\mathrm{HC}$ ). ${ }^{*}, P<0.05$ vs, the variable at the previous PEEP level; $\#, P<0.05$ vs. a variable at a PEEP of $3 \mathrm{cmH}_{2} \mathrm{O} . \alpha, P<0.05$ vs. Group HL within a PEEP; $\beta, P<0.05$ vs. Group HC within a PEEP; $\gamma$, $P<0.05$ vs. Group MC within a PEEP. 


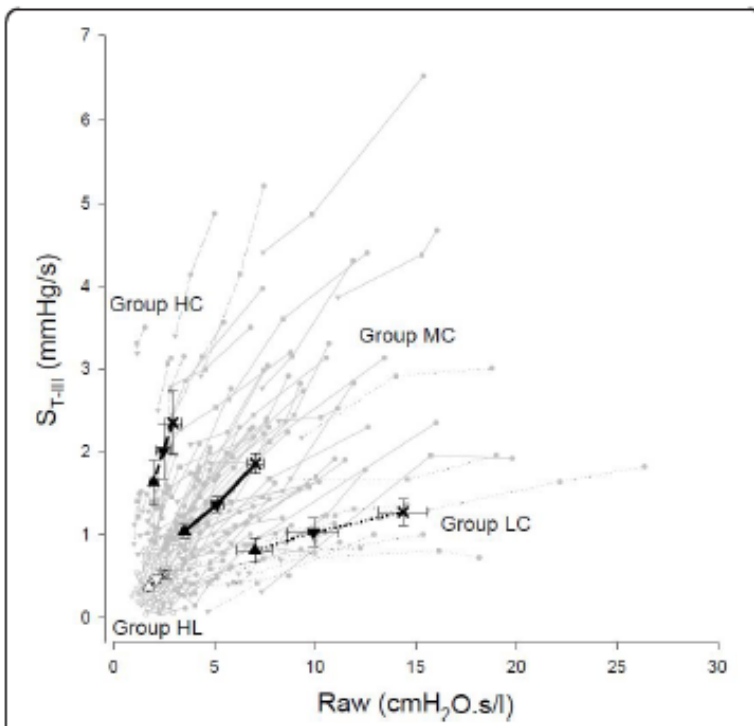

Figure 3 Relationship between forced oscillatory airway resistance (Raw) and phase III slope of time capnogram $\left(\mathrm{S}_{\mathrm{T}-\text { III }}\right)$ at PEEP levels of $3(\mathrm{X}), 6(\nabla)$ and $9 \mathrm{~cm} \mathrm{H}_{2} \mathrm{O}(\Delta)$ in patients with healthy lungs (Group $\mathrm{HL}$ ), and in patients with respiratory symptoms with dynamic respiratory compliance (Crs) in the lowest tenth percentile (Group LC), with Crs between the tenth and ninetieth percentile (Group MC) and with $\mathrm{Crs}$ above the ninetieth percentile (Group HC). Thin grey lines denote individual patients; thick black lines with symbols show group mean and SE values.

\section{Discussion}

The changes in airway and respiratory tissue mechanics were compared with capnogram third phase indices in a relatively large cohort of patients to clarify its monitoring value in mechanically ventilated patients. Capnogram third phase slopes expressed in the time domain or as a function of expired volume exhibited similar PEEP dependencies and distribution between the protocol groups. Detailed analysis of the time capnogram revealed a strong association between Raw and $\mathrm{S}_{\mathrm{T} \text {-III }}$ when the respiratory mechanics was altered by increasing PEEP, which was significantly affected by the degree of expiratory driving pressure of the respiratory system. Accordingly, grouping of the patients based on their Crs revealed that $\mathrm{i}$ ) the decrease in Raw with increasing PEEP was reflected in a sharp decrease in $\mathrm{S}_{\mathrm{T} \text {-III }}$ in patients with low Crs, ii) the increase in airway diameter with increasing PEEP was still reflected in a pronounced decrease in $\mathrm{S}_{\mathrm{T} \text {-III }}$ in patients with intermediate Crs, and iii) $\mathrm{S}_{\mathrm{T}-\text { III }}$ was insensitive to changes in airway caliber when the Crs was high.

The extent of emptying of lung compartments containing various $\mathrm{CO}_{2}$ concentrations during mechanical ventilation and the shape of the resulting capnogram are determined by the airway geometry (that is, the resistance) and the elastic recoil of the respiratory tissues (that is, the driving pressure). Whereas the former has been investigated extensively $[4,6,12,13,15]$, the importance of the latter factor remained unknown. While the time capnogram is most commonly used in clinical practice during mechanical ventilation, distinction of the second and third phases is not always trivial from the time domain analyses and this approach also excludes the consideration of the absolute concentration of $\mathrm{CO}_{2}$ in the expired gas. Therefore, we also performed volumetric capnography in a subgroup of patients and normalized the capnogram third phase slope. The similar picture of these different slopes and the significant correlation between them demonstrates that the $\mathrm{S}_{\mathrm{T}-\mathrm{III}}$ used in clinical practice can provide relevant information about lung emptying. With the aim of acquiring a general picture, the bedside Crs was used in the present study to group the patients. A strong correlation was earlier demonstrated between the respiratory elastance derived from $\mathrm{H}$ and the $\mathrm{Crs}$ [23], which justifies the choice of Crs as an appropriate indicator of the respiratory recoil.

We formed four groups with regard to the clinical symptoms (healthy lungs) and the Crs values (diseased lungs with low, medium or high $\mathrm{Crs}$ ). As expected, the variables reflecting resistive behavior were lowest in the Group HL and they had intermediate $\mathrm{H}$ and $\mathrm{Crs}$, permitting fast emptying of the relatively homogeneous lungs, which then results in low capnogram third phase slope, and good $\mathrm{PaO}_{2}$ (Figure 2). The increase of PEEP to $6 \mathrm{cmH}_{2} \mathrm{O}$ caused no further improvement. The slight, but significant decreases in Raw and $\mathrm{H}$ and increases in Crs and $\mathrm{PaO}_{2}$ at PEEP $9 \mathrm{cmH}_{2} \mathrm{O}$ may be a consequence of lung recruitment. The lack of decrease in the third phase slope indicates that this opening was relatively uniform in the lung periphery.

The patients in Group HC exhibited similar resistive properties to those in Group HL. However, the high Crs and low $\mathrm{H}$ may be a consequence of the loss of elastic recoil in the respiratory tissues, most probably due to emphysematous destruction, which was present in the vast majority of the patients in this group (Table 1). The presence of ventilation heterogeneities is also apparent from the highest third phase slope indices. These can be explained by the existence of peripheral lung units with different small airway calibers and local time constants, resulting in a heterogeneous working lung. This structure leads to gas compartments containing variable $\mathrm{CO}_{2}$ concentrations and also results in different local expiratory flows [11]. These phenomena contribute to the sequential emptying of the lung periphery in time, which then increases the time domain and volumetric $\mathrm{S}_{\mathrm{III}}$ values $[3-5,10,24]$. Since Raw reflects mainly the flow resistance of the central conducting airways $[19,23,25]$, this parameter is not able to detect such alterations in the presence of emphysematous changes [26]. This pathology 


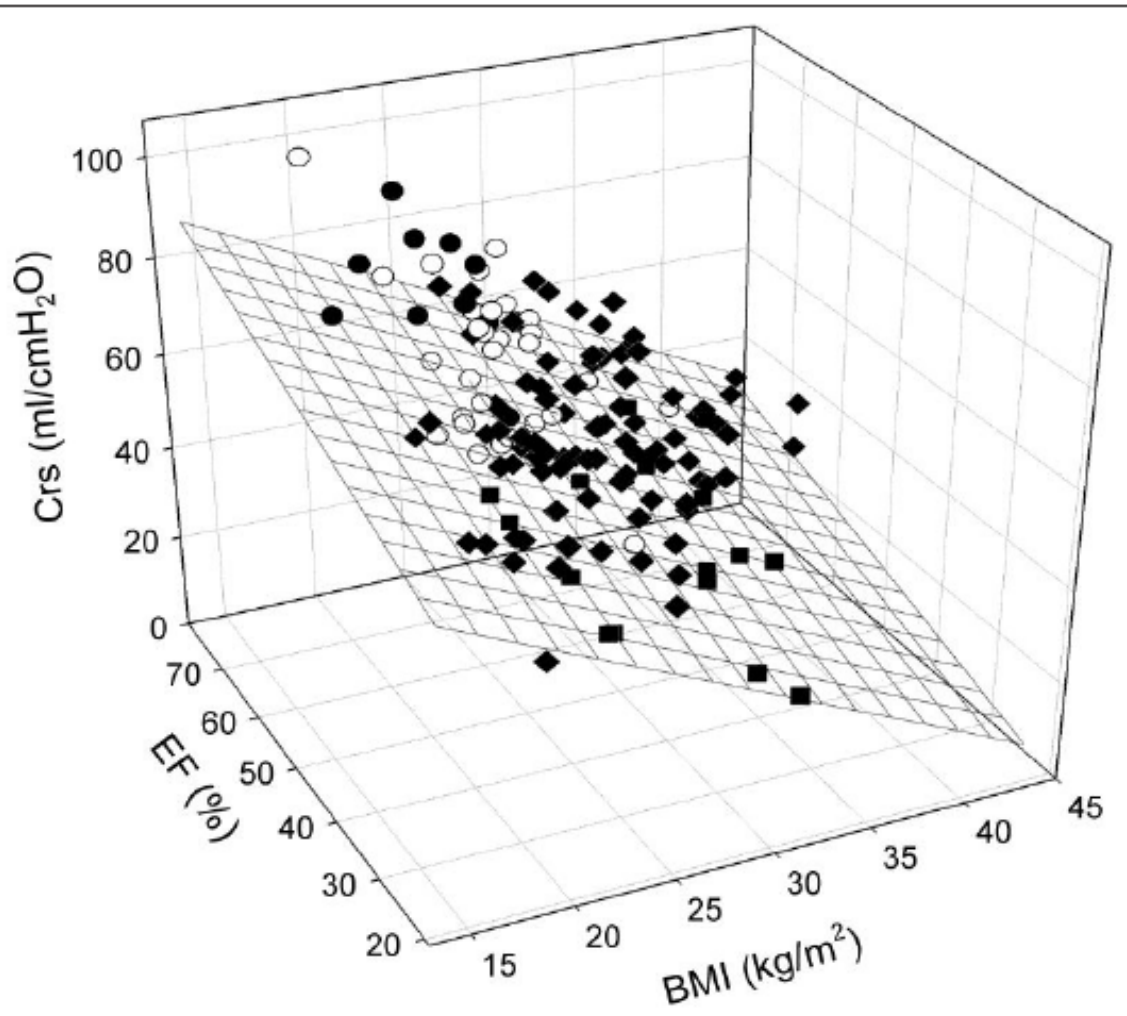

\section{Group HL \\ Hroup LC \\ Group MC \\ Group HC}

Figure 4 Effects of body mass index (BMI) and ejection fraction (EF) on dynamic respiratory compliance (Crs). The best fit plane is demonstrated with a mesh surface.

diminishes predominantly the expiratory flow, while the filling of the lung during mechanical ventilation may remain unaffected or even increased, explaining why $\mathrm{PaO}_{2}$ was close to normal. The elevation of PEEP in Group HC decreased G, which is a prerequisite of decreased ventilation heterogeneities with alveolar recruitment $[27,28]$, reflecting in lower capnogram third phase slopes, $\mathrm{H}$, higher Crs and better $\mathrm{PaO}_{2}$ [12]

The worst respiratory mechanics and the lowest $\mathrm{PaO}_{2}$ were observed in the patients in Group LC. Despite this striking difference, $\mathrm{S}_{\mathrm{III}}$ expressed in time or by volumetry did not differ significantly from those observed in Group HL. This leads to the important observation that even hypoxemia may be associated with a medium level of capnogram third phase slopes, which corresponds to the limited value of capnometry in the assessment of adequate blood oxygenation in this pathology $[29,30]$. These results can most probably be attributed to the presence of lung regions that remain closed throughout the entire ventilator cycle, leading to some relatively open and fairly uniform working lung units and other, permanently closed, atelectatic lung units. In other words, the closing capacity in these lungs is expected to be higher than the sum of the functional residual capacity and the tidal volume. The persistent lung volume loss with subsequent decrease in the overall airway cross-sectional area is probably reflected in the substantially elevated Raw. Since PEEP elevation may be able to reopen these atelectases, the involvement of these phenomena is substantiated by the most pronounced decreases in the mechanical parameters with increasing PEEP resulting in lower $\mathrm{S}_{\mathrm{II}}$ and elevated $\mathrm{PaO}_{2}$, which corresponds to earlier results on similar stiff lungs [10]. Our results confirm previous clinical observations $[9,12,25]$ that this pathophysiology can be triggered by obesity and/ or lung congestion arising from a poor EF (Table 1). Taking into account the individual and the combined 
effects of BMI and EF revealed that low EF or high BMI themselves may be responsible for the compromised Crs. However, the combination of such pathologies exerts additional detrimental effects that lower Crs even more dramatically (Figure 4).

Group MC comprised patients with pulmonary pathologies with an intermediate Crs, a cohort that can be characterized by somewhat elevated airway and respiratory tissue parameters, and ventilation heterogeneities reflected in abnormally high capnography slope characteristics at a PEEP of $3 \mathrm{cmH}_{2} \mathrm{O}$. This variable and the intermediate response to PEEP can be explained by concomitant presence of phenomena existing in Groups $\mathrm{HC}$ and $\mathrm{LC}$, that is, combined effects of expiratory flow limitation and persistent atelectases.

The overall Raw-S $\mathrm{S}_{\mathrm{T}-\mathrm{III}}$ relationship was not strong enough to predict the value of Raw from $\mathrm{S}_{\mathrm{T} \text {-III }}$ (Figure 3), in agreement with previous findings $[6,18]$. However, the changes in $\mathrm{S}_{\mathrm{T}-\mathrm{III}}$ within an individual patient were appropriate for an assessment or revealing trends of the altered Raw. It should be noted that the Raw-S $\mathrm{S}_{\mathrm{T}-\mathrm{III}}$ relationship within a patient was highly dependent on the elastic recoil of the respiratory system. In the case of a small Crs, a minor change in $\mathrm{S}_{\mathrm{T} \text {-III }}$ may reflect major alterations in airway patency. In contrast, large alterations in $\mathrm{S}_{\mathrm{T}-\mathrm{III}}$ may still be associated with small variations in Raw if Crs is high. This finding may explain the controversy in the literature concerning the presence or absence of a correlation between lung function parameters and capnogram indices $[11,13,15,16]$.

The limitations of this study relate to the possible presence of complex cardiopulmonary pathologies within a given patient. The coexistence of opposing factors such as emphysematous changes and a poor left ventricular function precludes identification of the individual effects of pulmonary diseases on the course of the capnogram. An additional aspect is that the surgery did not allow a more time-consuming randomization of the PEEP levels. However, care was taken to provide sufficient time following a change in conditions so that equilibrium was reached, similarly to that allowed following PEEP changes in severe COPD patients [27]. Another methodological limitation is related to the complex effects of PEEP including modification of the lung perfusion [2], increased functional residual capacity $[21,31]$, which may all bias the changes in $\mathrm{S}_{\mathrm{III}}$ and/or the mechanical parameters. However, our results are consistent even on PEEP $3 \mathrm{cmH}_{2} \mathrm{O}$ alone and the PEEP changes can be considered as reinforcement of the results and mechanisms that existed already at the lower PEEP. Auto-PEEP may be another important factor imposing a potential error with this bias being the most apparent in the patients with high Raw (that is, Group LC) or low driving pressure and compromised emptying of emphysematous destructed alveoli (that is, Group HC). Excluding the auto-PEEP would even enhance the Raw dependence with PEEP, since Raw would theoretically be even higher if auto-PEEP would have been ruled out. Another important feature of the present study is the use of $\mathrm{Crs}$ to separate the study groups. Since this parameter incorporates lung and chest wall properties, a separate assessment of which of these compartments are responsible for the altered elastic recoil of the respiratory system is not possible.

\section{Conclusions}

In summary, measurement of the respiratory mechanics and analysis of the capnogram slope demonstrated that changes in $\mathrm{S}_{\mathrm{III}}$ expressed in time or by volumetry provide useful information concerning alterations in airway caliber, but only within an individual patient. The assessment of $\mathrm{S}_{\mathrm{T}-\mathrm{III}}$ during mechanical ventilation may be of value for bedside monitoring of the airway resistance, but its sensitivity depends on the elastic recoil of the respiratory system. $\mathrm{S}_{\mathrm{T} \text {-III }}$ exhibits high sensitivity to detect changes in the airway resistance in case of high Crs, when the lung emptying is governed primarily by the small airway and alveolar geometry. In cases of stiff respiratory tissues, however, $\mathrm{S}_{\mathrm{T}-\mathrm{III}}$ displays low sensitivity in indicating changes in airway caliber, when the lung emptying is determined by the high elastic recoil and depends less on the small airway geometry. The relatively low $\mathrm{S}_{\mathrm{T} \text {-III }}$ may coincide with the compromised $\mathrm{PaO}_{2}$ in these patients, which suggests that a low $\mathrm{S}_{\mathrm{T}-\mathrm{III}}$ does not predict appropriate oxygenation. Thus, the shape of the capnogram should always be evaluated bedside in conjunction with $\mathrm{Crs}$. The joint assessment of the capnogram and the respiratory mechanics is of particular importance in clinical situations when patients with a high BMI and/or a compromised left ventricular function are anesthetized and ventilated.

\section{Key messages}

- The phase III slope of the capnogram evaluated in the time domain or by volumetry exhibits similar PEEP dependencies and distribution between the protocol groups formed on the basis of Crs.

- Crs significantly affects the sensitivity of the phase III slope of the capnogram in the time domain $\left(\mathrm{S}_{\mathrm{T}}\right.$ III) and by volumetry to airway dimensions.

- $\mathrm{S}_{\mathrm{T}-\mathrm{III}}$ detects changes in the airway resistance sensitively in cases of high $\mathrm{Crs}$.

- $\mathrm{S}_{\mathrm{T} \text {-III }}$ displays low sensitivity in cases of stiff respiratory tissues, in indicating changes in airway caliber.

- In conclusion, assessment of the capnogram shape should always be coupled with Crs when the airway resistance or oxygenation are evaluated. 


\section{Abbreviations}

BMt: body mass index; $\mathrm{CO}_{2}$ : carbon dioxide; COPD: chronic obstructive pulmonary disease; Crs: dynamic respiratory compliance; EF: ejection fraction; $\mathrm{FlO}_{2}$; fraction of inspired oxygen; $\mathrm{G}$; respiratory tissue damping; Group HC group of patients with high dynamic respiratory compliance; Group $\mathrm{HL}^{-}$ group of patients with healthy lungs; Group LC: group of patients with low dynamic respiratory compliance; Group MC group of patients with medium dynamic respiratory compl lance; $\mathrm{H}$. respiratory tissue elastance; law: airway inertance; $\mathrm{PaO}$; airway opening pressure: $\mathrm{PaO}_{2}$ : partial pressure of arterial oxygen; PEEP: positive end-expiratory pressure; Raw: airway resistance; Rrs: total respiratory resistance; $S_{\mathrm{i}}$ : slope of phase III of the capnogram; $S n_{\mathrm{F}}$ : normalized third phase slope of the expiratory capnogram in the time domain; Snv-s normalized volumetric third phase slope of the expiratory capnogram; $\mathrm{S}_{\mathrm{T}-\mathrm{i}}$ third phase slope of the expiratory capnogram in the time domain; $S_{-1}$ : volumetric third phase slope of the expiratory capnogram; $V$ : tracheal airflow; Zrs: input impedance of the respiratory system.

\section{Acknowledgements}

This work was supported by Hungarlan Basic Scientific Research Grant OTKA K81179. Ferenc Peták is supported by a Bolyai János Research Fellowship. The authors thank Prof. Zoltán Hantos for his highly pertinent advice and for his contribution in initiating volumetric measurements during the study.

\section{Author details}

'Department of Anesthesiology and Intensive Therapy, University of Szeged, 6 Semmelweis u., H-6720 Szeged. Hungary. ${ }^{2}$ Department of Medical Physics and Informatics, University of Szeged, 9 Korányi fasor, H-6720 Szeged, Hungary. ${ }^{3}$ Department of Anesthesiology, German Heart Center, 36 Lazarettstr., D-80636 Munich, Germany. ${ }^{\circ}$ Department of Cardiac Surgery, University of Szeged, 4 Pécsi u., H-6720 Szeged, Hungary.

\section{Authors' contributions}

$\mathrm{BB}$ conducted the design of the study and had a major role in data collection and drafting the manuscript. Cs7s and PNM helped with the data collection and analyses. BG performed the surgical preparation and helped with the measurements. $C D$ participated in the study design, data collection and helped with processing the data. FP supervised the data collection and analyses, contributed to the development of the study design and in the manuscript preparation. All authors read and approved the final manuscript.

\section{Competing interests}

The authors declare they have no competing interests in relation to this manuscript.

Received: 27 April 2012 Revised: 6 September 2012

Accepted: 2 October 2012 Published: 2 October 2012

\section{References}

1. Dubols $\mathrm{AB}, \mathrm{Britt} \mathrm{AG}$, Fenn WO: Alveolar $\mathrm{CO} 2$ during the respiratory cycle. J Appl Physiol 1952, 4:535-548.

2. Tusman G, Areta M, Climente C, Plit R, Suarez-Sipmann F, RodriguezNieto MJ, Peces-Barba G, Turchetto E, Bohm SH: Effect of pulmonary perfusion on the slopes of single-breath test of CO2.J Appl Physiol 2005, 99.650-655.

3. Crawford AB, Makowska M, Paiva M, Engel LA: Convection- and diffusiondependent ventilation maldistribution in normal subjects. J Appl Physio $1985,59.838-846$.

4. Stromberg NO, Gustafsson PM: Ventilation inhomogeneity assessed by nitrogen washout and ventilation-perfusion mismatch by capnography in stable and induced airway obstruction. Pediatr Puimono/ 2000, 29.94-102

5. Dutrieue B, Vanholsbeeck F, Verbanck S, Paiva M: A human acinar structure for simulation of realistic alveolar plateau slopes. J Appl Physol 2000, 89.1859-1867.

6. Blanch L, Lucangelo U, Lopez-Aguilar $\downarrow$, Fernandez R, Romero PV: Volumetric capnography in patients with acute lung injury: effects of positive end-expiratory pressure. Eur Respir J 1999, 13:1048-1054

7. Romero PV, Rodriguez B, de Olveira D, Blanch L, Manresa F: Volumetric capnography and chronic obstructive pulmonary disease staging. Int $J$ Chron Obstruat Puimon Dis 2007, 2381-391.
8. Veronez L, Moreira MM, Soares ST, Pereira MC, Ribeiro MA, Ribeiro JD, Terzi RG, Martins LC, Paschoal IA: Volumetric capnography for the evaluation of pulmonary disease in adult patients with cystic fibrosis and noncystic fibrosis bronchiectasis. Lung 2010, 188:263-268

9. Hoffbrand B: The expiratory capnogram: a measure of ventilationperfusion inequalities. Thorax 1966, 21:518-523.

10. Tusman G, Suarez-Sipmann F, Bohm SH, Borges JB, Hedenstiema G: Capnography reflects ventilation/perfusion distribution in a model of acute lung injury. Acta Anaextheriol Scand 2011, 55597-606.

11. Krauss B, Deykin A, Lam A, Ryoo JJ, Hampton DR, Schmitt PW, Falk JL: Capnogram shape in obstructive lung disease. Anesth Analg 2005, 100884888.

12. Bohm SH, Maisch S, von Sandersleben A, Thamm O, Passoni L, Martinez Arca 1 , Tusman G: The effects of lung recruitment on the Phase III slope of volumetric capnography in morbidly obese patients. Anesth Analg 2009, 109:151-159.

13. Nik Hisamuddin NA, Rashidi A, Chew KS, Kamaruddin J, Idzwan Z, Teo AH: Correlations between capnographic waveforms and peak flow meter measurement in emergency department management of asthma. int Emerg Med 2009, 2-83-89.

14. Thompson JE, Jaffe MB: Capnographic waveforms in the mechanically ventilated patient. Rexpir Care 2005, 50:100-108, discussion 108-109.

15. Yaron M, Padyk P. Hutsinpiller M, Cairns CB: Utility of the expiratory capnogram in the assessment of bronchospasm. Ann Emerg Med 1996 , 28:403-407.

16. You B, Peslin R, Duvivier C, Vu VD, Grilliat JP. Expiratory capnography in asthma: evaluation of various shape indices. Eur Respir J 1994, 7:318-32

17. Kars AH, Bogaard JM, Stijnen T, de Vries J, Verbraak AF, Hilvering C: Dead space and slope indices from the expiratory carbon dioxide tensionvolume curve. Eur Respir J 1997, 10:1829-1836.

18. Blanch L, Fernandez R, Saura P, Baigorri F, Artigas A: Relationship between expired capnogram and respiratory system resistance in critically ill patients during total ventilatory support. Chest 1994, 105:219-223.

19. Babik B, Asctalos T, Petak F, Deak Zl, Hantos Z: Changes in respiratory mechanics during cardiac surgery. Anexth Analg 2003, 96:1280-1287.

20. Hantos Z, Daroczy B, Suk B, Nagy S, Fredberg JJ: Input impedance and peripheral inhomogeneity of dog lungs. J Appl Physio/ 1992, 72:168-178.

21. Ream RS, Schreiner MS, Neff JD, McRae KM, Jawad AE, Scherer PW, Neufeld GR: Volumetric capnography in children. Influence of growth on the alveolar plateau slope. Anesthesiology 1995, 82.64.73.

22. Tsouklas NM, Tannous Z, Wison AF, George SC: Single-exhalation profiles of $\mathrm{NO}$ and $\mathrm{CO} 2$ in humans: effect of dynamically changing flow rate. J Appl Physiol 1998, 85.642-652

23. Babik B, Petak F, Astalos T, Deak Zl, Bogats G, Hantos Z Components of respiratory resistance monitored in mechanically ventilated patients. Eur Respir J 2002, 20:1538-1544.

24. van Meerten RJ: Expiratory gas concentration curves for examination of uneven distribution of ventilation and perfusion in the lung. First communication: theory. Respiration 1970, 27:552-564.

25. Albu G, Babik B, Kesmarky K, Balazs M, Hantos Z, Petak F: Changes in airway and respiratory tissue mechanics after cardiac surgery. Ann Thorac Surg 2010, 89:1218-1226.

26. Tolnai J, Szabari MV, Albu G, Mar BA, Parameswaran H, Bartolak-Sukj E, Suk B, Hantos Z: Functional and morphological assessment of early impairment of airway function in a rat model of emphysema. J Appl Physiol 2012, 112:1932-1939.

27. Lorx A, Szabo B, Hercsuth M, Penzes I, Hantos Z: Low-frequency assessment of airway and tissue mechanics in ventilated COPD patients. J Appl Physiol 2009, 107:1884-1892

28. Lutchen KR, Hantos Z, Petak F, Adamicza A, Suki B. Airway inhomogeneities contribute to apparent lung tissue mechanics during constriction. J App/ Physiol 1996, 80:1841-1849.

29. Napolitano LM: Capnography in citical care: accurate assessment of ARDS therapy? Cnit Care Med 1999, 27:862-86:

30. Morley TF, Gaimo 1 , Maroszan E, Bermingham J, Gordon R, Griesback R, Zappasodi Sl, Gudice JC: Use of capnography for assessment of the adequacy of alveolar ventilation during weaning from mechanical ventilation. Am Rev Respir Dis 1993, 148:339-344.

31. Tusman G, Bohm SH, Suarez-Sipmann F, Scandurra A, Hedenstiema G: Lung recruitment and positive end-expiratory pressure have different effects 
Babik et al. Gritical Care 2012, 16:R177 http://ccorum.com/content/16/5/R177

on $\mathrm{CO} 2$ elimination in healthy and sick lungs. Anesth Analg 2010, 111968-977.

doi: $10.1186 / \mathrm{cc} 11659$

Gite this article as: Babik et af: Effects of respiratory mechanics on the capnogram phases: importance of dynamic compliance of the respiratory system. Critical Care 2012 16R177. 